\title{
Dearomatization-Rearomatization Strategy for Reductive Cross-Coupling of Indoles with Ketones in Water
}

\author{
Zemin Wang, ${ }^{\dagger}$ Huiying Zeng ${ }^{*} \dagger$ and Chao-Jun $\mathrm{Li}^{*}{ }^{* \dagger}$ \\ ${ }^{\dagger}$ The State Key Laboratory of Applied Organic Chemistry, Lanzhou University, 222 \\ Tianshui Road, Lanzhou, 730000, P. R. China \\ Department of Chemistry and FQRNT Centre for Green Chemistry and Catalysis, \\ McGill University, 801 Sherbrooke St. West, Montreal, Quebec H3A 0B8, Canada \\ *Corresponding Authors: zenghy@1zu.edu.cn and cj.li@mcgill.ca
}

\section{Table of Contents}

I . General information ........................................... SError! Bookmark not defined.

II . Optimization of reaction conditions ................... SError! Bookmark not defined.

III. General procedure ...........................................................................S4

IV . Characterization data .......................................................................S4

V . Copies of ${ }^{1} \mathrm{H}$ NMR, ${ }^{19}$ F NMR and ${ }^{13} \mathrm{C}$ NMR.................................................S18 


\section{General information}

All reagents were purchased from the suppliers and used without further purification. ${ }^{1} \mathrm{H},{ }^{19} \mathrm{~F}$ and ${ }^{13} \mathrm{C}$ NMR spectra were recorded on a $400 \mathrm{MHz}$ and $600 \mathrm{MHz} \mathrm{NMR}$ spectra meter in $\mathrm{CDCl}_{3}$ at room temperature. The chemical shifts are referenced to internal TMS. Silica gel (300-400 mesh) was used for flash column chromatograph, eluting (unless otherwise stated) with an ethyl acetate/hexane mixture. HRMS analyses were made by Lanzhou University by means of ESI. Melting points were measured on micro melting point apparatus and uncorrected. All solvents were purified and dried by standard techniques.

\section{Optimization of reaction conditions}

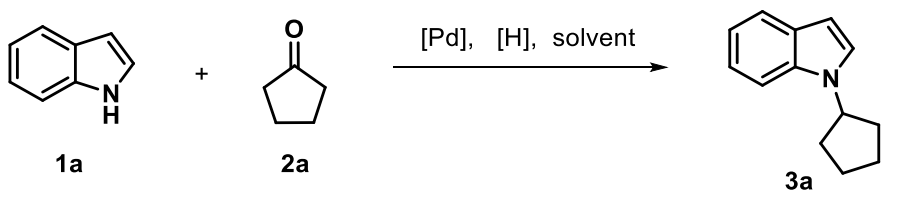

1) Effects of catalysts on yields ${ }^{a}$

\begin{tabular}{ccc}
\hline Entry & Conditions & Yield (\%) \\
\hline 1 & $\mathrm{Pd} / \mathrm{C}(10 \mathrm{wt} \%)$ & 44 \\
2 & $\mathrm{Pd}\left(\mathrm{PPh}_{3}\right)_{4}$ & n.p. \\
3 & $\mathrm{PdCl}_{2}$ & 9 \\
4 & $\mathrm{Pd}(\mathrm{OAc})_{2}$ & 6 \\
5 & $\mathrm{Pd}(\mathrm{OH})_{2}$ & 8 \\
6 & $\mathrm{Pd}(\mathrm{OH})_{2} / \mathrm{C}(5 \mathrm{wt} \%$ of Alfa $)$ & 84 \\
7 & $\mathrm{Pd}(\mathrm{OH})_{2} / \mathrm{C}(10 \mathrm{wt} \%$ of Energy $)$ & 59 \\
9 & $\mathrm{Pd}(\mathrm{OH})_{2} / \mathrm{C}(20 \mathrm{wt} \%$ of Acros $)$ & 48 \\
\hline
\end{tabular}

${ }^{a}$ Reaction conditions: indole (0.2 mmol), cyclopentanone ( $\left.0.5 \mathrm{mmol}\right),[\mathrm{Pd}](10 \mathrm{~mol} \%)$, $\mathrm{HCO}_{2} \mathrm{Na}(1.0 \mathrm{mmol}), \mathrm{H}_{2} \mathrm{O}(1 \mathrm{~mL})$ under Ar at $100{ }^{\circ} \mathrm{C}$ for $24 \mathrm{~h}$.

2) Effects of $\mathrm{HCO}_{2} \mathrm{Na}$ amount on yields ${ }^{a}$

\begin{tabular}{ccc}
\hline Entry & Conditions & Yield (\%) \\
\hline 1 & $\mathrm{HCO}_{2} \mathrm{Na}(1.0 \mathrm{mmol})$ & 84 \\
2 & $\mathrm{HCO}_{2} \mathrm{Na}(0.5 \mathrm{mmol})$ & 87 \\
3 & $\mathrm{HCO}_{2} \mathrm{Na}(0.4 \mathrm{mmol})$ & 81 \\
\hline
\end{tabular}

${ }^{a}$ Reaction conditions: indole $(0.2 \mathrm{mmol})$, cyclopentanone $(0.5 \mathrm{mmol}), \mathrm{Pd}(\mathrm{OH})_{2} / \mathrm{C}(5$ wt $\%$ of Alfa) $(10 \mathrm{~mol} \%), \mathrm{HCO}_{2} \mathrm{Na}, \mathrm{H}_{2} \mathrm{O}(1 \mathrm{~mL})$ under Ar at $100{ }^{\circ} \mathrm{C}$ for $24 \mathrm{~h}$.

3) Effects of hydrogen sources on yields ${ }^{a}$ 


\begin{tabular}{ccc}
\hline Entry & Conditions & Yield (\%) \\
\hline 1 & $\mathrm{HCO}_{2} \mathrm{Na}$ & 87 \\
2 & $\mathrm{HCO}_{2} \mathrm{Li} \cdot \mathrm{H}_{2} \mathrm{O}$ & 68 \\
3 & $\mathrm{HCO}_{2} \mathrm{~K}$ & 90 \\
4 & $\mathrm{HCO}_{2} \mathrm{Cs}$ & 80 \\
5 & $\mathrm{HCO}_{2} \mathrm{NH}_{4}$ & 83 \\
6 & $\mathrm{HCO}_{2} \mathrm{H}$ & 71 \\
7 & $\mathrm{H}_{2}$ & 63 \\
\hline
\end{tabular}

${ }^{a}$ Reaction conditions: indole $(0.2 \mathrm{mmol})$, cyclopentanone $(0.5 \mathrm{mmol}), \mathrm{Pd}(\mathrm{OH})_{2} / \mathrm{C}(5$ wt $\%$ of Alfa) $(10 \mathrm{~mol} \%),[\mathrm{H}](0.5 \mathrm{mmol}), \mathrm{H}_{2} \mathrm{O}(1 \mathrm{~mL})$ under $\mathrm{Ar}$ at $100{ }^{\circ} \mathrm{C}$ for $24 \mathrm{~h}$.

4) Effects of cyclopentanone amount on yields ${ }^{a}$

\begin{tabular}{ccc}
\hline Entry & Conditions & Yield (\%) \\
\hline 1 & $0.4 \mathrm{mmol}$ & 65 \\
2 & $0.5 \mathrm{mmol}$ & 90 \\
3 & $0.6 \mathrm{mmol}$ & 94 \\
\hline
\end{tabular}

${ }^{a}$ Reaction conditions: indole $(0.2 \mathrm{mmol})$, cyclopentanone, $\mathrm{Pd}(\mathrm{OH})_{2} / \mathrm{C}(5 \mathrm{wt} \%$ of Alfa $)$ (10 mol\%), $\mathrm{HCO}_{2} \mathrm{~K}(0.5 \mathrm{mmol}), \mathrm{H}_{2} \mathrm{O}(1 \mathrm{~mL})$ under $\mathrm{Ar}$ at $100{ }^{\circ} \mathrm{C}$ for $24 \mathrm{~h}$.

5) Effects of reaction temperature ${ }^{a}$

\begin{tabular}{ccc}
\hline Entry & Conditions & Yield (\%) \\
\hline 1 & $70^{\circ} \mathrm{C}$ & 73 \\
2 & $100^{\circ} \mathrm{C}$ & 94 \\
3 & $130^{\circ} \mathrm{C}$ & 51 \\
\hline
\end{tabular}

${ }^{a}$ Reaction conditions: indole $(0.2 \mathrm{mmol})$, cyclopentanone $(0.6 \mathrm{mmol}), \mathrm{Pd}(\mathrm{OH})_{2} / \mathrm{C}(5$ wt $\%$ of Alfa) $(10 \mathrm{~mol} \%), \mathrm{HCO}_{2} \mathrm{~K}(0.5 \mathrm{mmol}), \mathrm{H}_{2} \mathrm{O}(1 \mathrm{~mL})$ under Ar for $24 \mathrm{~h}$.

6) Effects of solvents ${ }^{a}$

\begin{tabular}{ccc}
\hline Entry & Conditions & Yield (\%) \\
\hline 1 & $\mathrm{H}_{2} \mathrm{O}$ & 94 \\
2 & $i$-PrOH & trace \\
3 & $\mathrm{PhMe}$ & $13 \%$ \\
4 & $\mathrm{THF}$ & n.p. \\
5 & EtOAc & n.p. \\
\hline
\end{tabular}

${ }^{a}$ Reaction conditions: indole $(0.2 \mathrm{mmol})$, cyclopentanone $(0.6 \mathrm{mmol}), \mathrm{Pd}(\mathrm{OH})_{2} / \mathrm{C}(5$ $\mathrm{wt} \%$ of Alfa) $(10 \mathrm{~mol} \%), \mathrm{HCO}_{2} \mathrm{~K}(0.5 \mathrm{mmol})$, solvent $(1 \mathrm{~mL})$ under Ar for $24 \mathrm{~h}$.

7) Effects of reaction concentration on yields ${ }^{a}$ 


\begin{tabular}{ccc}
\hline Entry & Conditions & Yield (\%) \\
\hline 1 & $1 \mathrm{~mL} \mathrm{H}_{2} \mathrm{O}$ & 94 \\
2 & $0.5 \mathrm{~mL} \mathrm{H}_{2} \mathrm{O}$ & 99 \\
3 & $2 \mathrm{~mL} \mathrm{H}_{2} \mathrm{O}$ & 92 \\
\hline
\end{tabular}

${ }^{a}$ Reaction conditions: indole $(0.2 \mathrm{mmol})$, cyclopentanone $(0.6 \mathrm{mmol}), \mathrm{Pd}(\mathrm{OH})_{2} / \mathrm{C}$

$\left(5 \mathrm{wt} \%\right.$ of Alfa) $(10 \mathrm{~mol} \%), \mathrm{HCO}_{2} \mathrm{~K}(0.5 \mathrm{mmol}), \mathrm{H}_{2} \mathrm{O}$ under $\mathrm{Ar}$ at $100{ }^{\circ} \mathrm{C}$ for $24 \mathrm{~h}$.

Note: Yields were determined by ${ }^{1} \mathrm{H}$ NMR with nitromethane as internal standard.

\section{General procedure}

\section{1) The method by using $\mathrm{HCO}_{2} \mathrm{~K}$.}

$\mathrm{Pd}(\mathrm{OH})_{2} / \mathrm{C}(5 \mathrm{wt} \%$ of Alfa, $56 \mathrm{mg}, 10 \mathrm{~mol} \%$ based on Pd contents), indole $(23.4 \mathrm{mg}, 0.2 \mathrm{mmol})$ and potassium formate $(42.0 \mathrm{mg}, 0.5 \mathrm{mmol})$ were added into a test tube $(20 \mathrm{~mL})$ charged with a magnetic stir bar. After three cycles of evacuation/backfilling sequence with argon, cyclopentanone $(53 \mu \mathrm{L}, 0.6$ mmol $)$ and distilled water $(0.5 \mathrm{~mL})$ were added. Then the reaction mixture was stirred in a preheated oil bath at $100^{\circ} \mathrm{C}$ for $24 \mathrm{~h}$. The reaction system was cooled to room temperature. The mixture was extracted with dichloromethane $(3 \times 20 \mathrm{~mL})$ and the combined organic extracts were washed by brine, dried over $\mathrm{Na}_{2} \mathrm{SO}_{4}$, filtered, concentrated, and purified by flash chromatography on silica gel (PE/EA: $100 / 1$ to $15 / 1$ ) to give product 3 .

\section{2) The method by using $\mathrm{HCO}_{2} \mathrm{H}$.}

$\mathrm{Pd}(\mathrm{OH})_{2} / \mathrm{C}(5 \mathrm{wt} \%$ of Alfa, $56 \mathrm{mg}, 10 \mathrm{~mol} \%$ based on Pd contents) and indole $(23.4 \mathrm{mg}, 0.2 \mathrm{mmol})$ were added into a test tube $(20 \mathrm{~mL})$ charged with a magnetic stir bar. After three cycles of evacuation/backfilling sequence with argon, ketone $(0.6 \mathrm{mmol})$, distilled water $(0.5 \mathrm{~mL})$ and formic acid ( $88 \mathrm{wt} \%, 18 \mu \mathrm{L}, 0.4 \mathrm{mmol})$ were added. Then the reaction was stirred in a preheated oil bath at $100{ }^{\circ} \mathrm{C}$ for $24 \mathrm{~h}$. The reaction system was cooled to room temperature. The mixture was extracted with dichloromethane $(3 \times 20 \mathrm{~mL})$ and the combined organic extracts were washed by brine, dried over $\mathrm{Na}_{2} \mathrm{SO}_{4}$, filtered, concentrated, and purified by flash chromatography on silica gel (PE/EA: 100/1 to $15 / 1)$ to give product 3 .

\section{3) The method for $1 \mathrm{mmol}$ scale.}

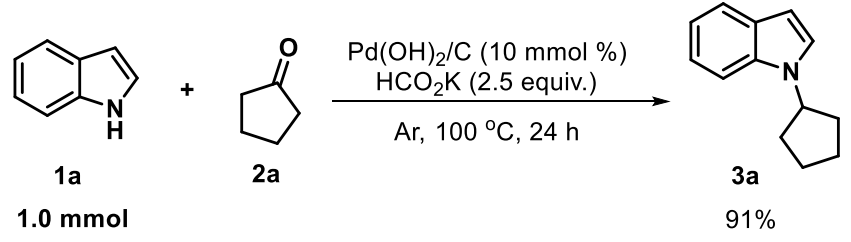

$\mathrm{Pd}(\mathrm{OH})_{2} / \mathrm{C}(5 \mathrm{wt} \%$ of Alfa, $280 \mathrm{mg}, 10 \mathrm{~mol} \%$ based on Pd contents), indole $(117 \mathrm{mg}, 1.0 \mathrm{mmol})$ and potassium formate $(210 \mathrm{mg}, 2.5 \mathrm{mmol})$ were added into a Schlenk tube $(25 \mathrm{~mL})$ charged with a magnetic stir bar. After three cycles of evacuation/backfilling sequence with argon, cyclopentanone $(265 \mu \mathrm{L}, 3.0 \mathrm{mmol})$ and distilled water $(2.5 \mathrm{~mL})$ were added. Then the reaction mixture was stirred in a preheated oil bath at $100{ }^{\circ} \mathrm{C}$ for $24 \mathrm{~h}$. The reaction system was cooled to room temperature. The mixture 
was extracted with dichloromethane $(3 \times 30 \mathrm{~mL})$ and the combined organic extracts were washed by brine, dried over $\mathrm{Na}_{2} \mathrm{SO}_{4}$, filtered, concentrated, and purified by flash chromatography on silica gel (PE/EA: 100/1) to give product 3a $169 \mathrm{mg}, 91 \%$ yield.

\section{Characterization data}

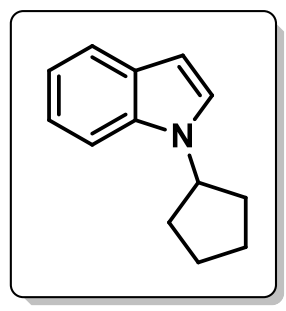

1-cyclopentylindole (3a)

Colorless oil; $36.0 \mathrm{mg}, 97 \%$ yield.

${ }^{1} \mathbf{H}$ NMR $\left(400 \mathrm{MHz}, \mathrm{CDCl}_{3}\right) \delta 7.62(\mathrm{~d}, J=7.8 \mathrm{~Hz}, 1 \mathrm{H}), 7.40(\mathrm{~d}, J=8.3 \mathrm{~Hz}, 1 \mathrm{H}), 7.22-7.17(\mathrm{~m}, 2 \mathrm{H})$, $7.12-7.07(\mathrm{~m}, 1 \mathrm{H}), 6.49(\mathrm{~d}, J=3.2 \mathrm{~Hz}, 1 \mathrm{H}), 4.83-4.73(\mathrm{~m}, 1 \mathrm{H}), 2.24-2.14(\mathrm{~m}, 2 \mathrm{H}), 1.98-1.84(\mathrm{~m}$, 4H), $1.80-1.70(\mathrm{~m}, 2 \mathrm{H})$.

${ }^{13} \mathrm{C}$ NMR $\left(101 \mathrm{MHz}, \mathrm{CDCl}_{3}\right) \delta 136.0,128.6,124.4,121.1,120.8,119.2,109.8,100.8,56.8,32.5,24.0$. IR (KBr) : 2959, 2873, 1461, 1314, 1226, $739 \mathrm{~cm}^{-1}$.

HRMS (ESI): Calcd for $\mathrm{C}_{13} \mathrm{H}_{16} \mathrm{~N}(\mathrm{M}+\mathrm{H})^{+}:$186.1277; Found: 186.1279.

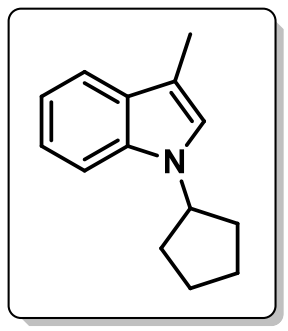

\section{1-cyclopentyl-3-methylindole (3b)}

Colorless oil; $38.8 \mathrm{mg}, 97 \%$ yield.

${ }^{1} \mathbf{H}$ NMR $\left(400 \mathrm{MHz}, \mathrm{CDCl}_{3}\right) \delta 7.56(\mathrm{~d}, J=7.8 \mathrm{~Hz}, 1 \mathrm{H}), 7.35(\mathrm{~d}, J=8.3 \mathrm{~Hz}, 1 \mathrm{H}), 7.19(\mathrm{dd}, J=8.0,7.2$ Hz, 1H), 7.09 (t, J=7.4 Hz, 1H), $6.96(\mathrm{~s}, 1 \mathrm{H}), 4.78-4.69(\mathrm{~m}, 1 \mathrm{H}), 2.33(\mathrm{~s}, 3 \mathrm{H}), 2.20-2.11(\mathrm{~m}, 2 \mathrm{H})$, $1.94-1.84(\mathrm{~m}, 4 \mathrm{H}), 1.75-1.71(\mathrm{~m}, 2 \mathrm{H})$.

${ }^{13} \mathbf{C}$ NMR (101 MHz, $\left.\mathrm{CDCl}_{3}\right) \delta 136.3,128.6,122.1,121.0,118.9,118.4,110.0,109.5,56.5,32.6,24.1$, 9.7 .

IR (KBr) : 2961, 2871, 1463, 1358, 1231, $736 \mathrm{~cm}^{-1}$.

HRMS (ESI): Calcd for $\mathrm{C}_{14} \mathrm{H}_{18} \mathrm{~N}(\mathrm{M}+\mathrm{H})^{+}:$: 200.1434; Found: 200.1438.

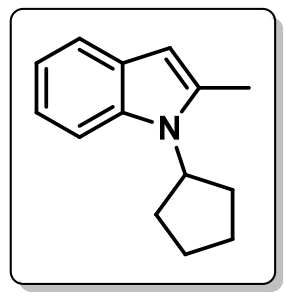




\section{1-cyclopentyl-2-methylindole (3c)}

Colorless oil; $21.1 \mathrm{mg}, 53 \%$ yield.

${ }^{1} \mathbf{H}$ NMR $\left(400 \mathrm{MHz}, \mathrm{CDCl}_{3}\right) \delta 7.61-7.54(\mathrm{~m}, 1 \mathrm{H}), 7.45-7.40(\mathrm{~m}, 1 \mathrm{H}), 7.16-7.11(\mathrm{~m}, 1 \mathrm{H}), 7.10-$ $7.08(\mathrm{~m}, 1 \mathrm{H}), 6.26(\mathrm{~s}, 1 \mathrm{H}), 4.85-4.75(\mathrm{~m}, 1 \mathrm{H}), 2.49(\mathrm{~s}, 3 \mathrm{H}), 2.37-2.28(\mathrm{~m}, 2 \mathrm{H}), 2.11-2.04(\mathrm{~m}, 4 \mathrm{H})$, $1.86-1.78(\mathrm{~m}, 2 \mathrm{H})$.

${ }^{13}$ C NMR $\left(101 \mathrm{MHz}, \mathrm{CDCl}_{3}\right) \delta$ 136.9, 134.6, 128.7, 119.9, 119.8, 118.8, 110.8, 100.0, 56.0, 30.0, 25.3, 13.8 .

IR (KBr) : 2955, 2873, 1459, 1412, 1343, $747 \mathrm{~cm}^{-1}$.

HRMS (ESI): Calcd for $\mathrm{C}_{14} \mathrm{H}_{18} \mathrm{~N}(\mathrm{M}+\mathrm{H})^{+}:$200.1434; Found: 200.1438 .

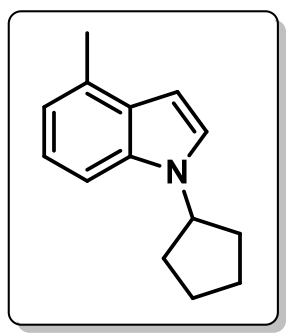

\section{1-cyclopentyl-4-methylindole (3d)}

Colorless oil; $34.4 \mathrm{mg}, 86 \%$ yield.

${ }^{1} \mathbf{H}$ NMR $\left(400 \mathrm{MHz}, \mathrm{CDCl}_{3}\right) \delta 7.25(\mathrm{~d}, J=8.3 \mathrm{~Hz}, 1 \mathrm{H}), 7.19(\mathrm{~d}, J=3.2 \mathrm{~Hz}, 1 \mathrm{H}), 7.11(\mathrm{t}, J=7.7 \mathrm{~Hz}$, $1 \mathrm{H}), 6.90(\mathrm{dd}, J=7.0,0.6 \mathrm{~Hz}, 1 \mathrm{H}), 6.51(\mathrm{~d}, J=3.2 \mathrm{~Hz}, 1 \mathrm{H}), 4.83-4.70(\mathrm{~m}, 1 \mathrm{H}), 2.55(\mathrm{~s}, 3 \mathrm{H}), 2.23-$ $2.15(\mathrm{~m}, 2 \mathrm{H}), 1.97-1.85(\mathrm{~m}, 4 \mathrm{H}), 1.77-1.74(\mathrm{~m}, 2 \mathrm{H})$.

${ }^{13}$ C NMR $\left(101 \mathrm{MHz}, \mathrm{CDCl}_{3}\right) \delta$ 135.7, 130.3, 128.4, 123.8, 121.3, 119.4, 107.4, 99.3, 57.0, 32.5, 24.0, 18.8 .

IR (KBr) : 2959, 2871, 1489, 1457, 1239, 743, $710 \mathrm{~cm}^{-1}$.

HRMS (ESI): Calcd for $\mathrm{C}_{14} \mathrm{H}_{18} \mathrm{~N}(\mathrm{M}+\mathrm{H})^{+}:$200.1434; Found: 200.1435 .

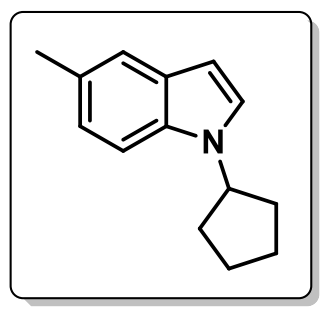

\section{1-cyclopentyl-5-methylindole (3e)}

Colorless oil; $35.7 \mathrm{mg}, 90 \%$ yield.

${ }^{1} \mathbf{H}$ NMR $\left(400 \mathrm{MHz}, \mathrm{CDCl}_{3}\right) \delta 7.41(\mathrm{~s}, 1 \mathrm{H}), 7.28(\mathrm{~d}, J=8.4 \mathrm{~Hz}, 1 \mathrm{H}), 7.14(\mathrm{~d}, J=3.0 \mathrm{~Hz}, 1 \mathrm{H}), 7.02(\mathrm{~d}$, $J=8.4 \mathrm{~Hz}, 1 \mathrm{H}), 6.40(\mathrm{~d}, J=3.1 \mathrm{~Hz}, 1 \mathrm{H}), 4.78-4.69(\mathrm{~m}, 1 \mathrm{H}), 2.44(\mathrm{~s}, 3 \mathrm{H}), 2.22-2.12(\mathrm{~m}, 2 \mathrm{H}), 1.96-$ $1.82(\mathrm{~m}, 4 \mathrm{H}), 1.80-1.69(\mathrm{~m}, 2 \mathrm{H})$.

${ }^{13} \mathrm{C}$ NMR $\left(101 \mathrm{MHz}, \mathrm{CDCl}_{3}\right) \delta 134.4,128.8,128.3,124.5,122.7,120.4,109.5,100.2,56.9,32.5,24.0$, 21.4 .

IR (KBr) : 2961, 2871, 1481, 1224, 788, $758 \mathrm{~cm}^{-1}$.

HRMS (ESI): Calcd for $\mathrm{C}_{14} \mathrm{H}_{18} \mathrm{~N}(\mathrm{M}+\mathrm{H})^{+}:$200.1434; Found: 200.1434 . 


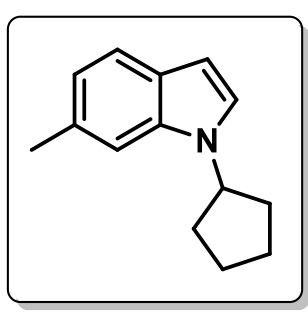

1-cyclopentyl-6-methylindole (3f)

Colorless oil; $28.4 \mathrm{mg}, 71 \%$ yield.

${ }^{1} \mathbf{H}$ NMR $\left(400 \mathrm{MHz}, \mathrm{CDCl}_{3}\right) \delta 7.56(\mathrm{~d}, J=8.0 \mathrm{~Hz}, 1 \mathrm{H}), 7.26(\mathrm{~s}, 1 \mathrm{H}), 7.18(\mathrm{~d}, J=3.2 \mathrm{~Hz}, 1 \mathrm{H}), 6.99(\mathrm{dd}$, $J=8.0,0.9 \mathrm{~Hz}, 1 \mathrm{H}), 6.50(\mathrm{dd}, J=3.2,0.6 \mathrm{~Hz}, 1 \mathrm{H}), 4.81(\mathrm{dd}, J=13.9,6.9 \mathrm{~Hz}, 1 \mathrm{H}), 2.55(\mathrm{~s}, 3 \mathrm{H}), 2.29-$ $2.22(\mathrm{~m}, 2 \mathrm{H}), 2.02-1.91(\mathrm{~m}, 4 \mathrm{H}), 1.84-1.81(\mathrm{~m}, 2 \mathrm{H})$.

${ }^{13}$ C NMR $\left(101 \mathrm{MHz}, \mathrm{CDCl}_{3}\right) \delta$ 136.5, 130.8, 126.3, 123.8, 121.0, 120.4, 109.7, 100.7, 56.7, 32.5, 24.0, 22.0 .

IR (KBr) : 2961, 2872, 1461, 1321, 1226, 799, $710 \mathrm{~cm}^{-1}$.

HRMS (ESI): Calcd for $\mathrm{C}_{14} \mathrm{H}_{18} \mathrm{~N}(\mathrm{M}+\mathrm{H})^{+}:$200.1434; Found: 200.1434 .

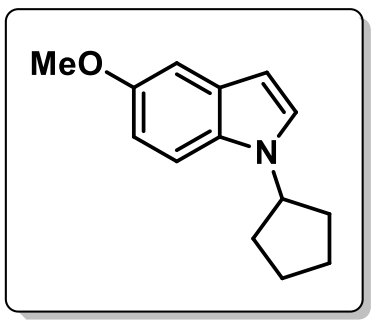

\section{1-cyclopentyl-5-methoxyindole (3g)}

Colorless oil; $40.9 \mathrm{mg}, 95 \%$ yield.

${ }^{1} \mathbf{H}$ NMR $\left(400 \mathrm{MHz}, \mathrm{CDCl}_{3}\right) \delta 7.28(\mathrm{~d}, J=8.9 \mathrm{~Hz}, 1 \mathrm{H}), 7.16(\mathrm{~d}, J=3.2 \mathrm{~Hz}, 1 \mathrm{H}), 7.08(\mathrm{~d}, J=2.4 \mathrm{~Hz}$, $1 \mathrm{H}), 6.86(\mathrm{dd}, J=8.9,2.5 \mathrm{~Hz}, 1 \mathrm{H}), 6.41(\mathrm{~d}, J=3.1 \mathrm{~Hz}, 1 \mathrm{H}), 4.76-4.68(\mathrm{~m}, 1 \mathrm{H}), 3.84(\mathrm{~s}, 3 \mathrm{H}), 2.22-$ $2.13(\mathrm{~m}, 2 \mathrm{H}), 1.96-1.82(\mathrm{~m}, 4 \mathrm{H}), 1.80-1.69(\mathrm{~m}, 2 \mathrm{H})$.

${ }^{13} \mathrm{C}$ NMR $\left(101 \mathrm{MHz}, \mathrm{CDCl}_{3}\right) \delta 153.8,131.4,128.8,125.0,111.5,110.5,102.2,100.3,57.0,55.8,32.5$, 24.0.

IR (KBr) : 2953, 2875, 2830, 1483, 1451, 1241, 1151, 1034, 801, $713 \mathrm{~cm}^{-1}$.

HRMS (ESI): Calcd for $\mathrm{C}_{14} \mathrm{H}_{18} \mathrm{NO}(\mathrm{M}+\mathrm{H})^{+}: 216.1383$; Found: 216.1382 .

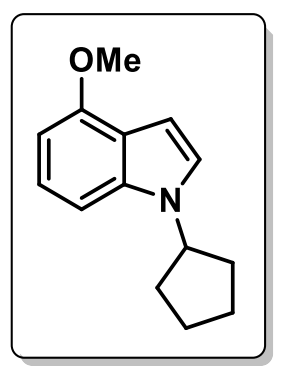

1-cyclopentyl-4-methoxyindole $(3 \mathrm{~h})$

Colorless oil; $33.2 \mathrm{mg}$, 77\% yield.

${ }^{1} \mathbf{H}$ NMR $\left(400 \mathrm{MHz}, \mathrm{CDCl}_{3}\right) \delta 7.13(\mathrm{dd}, J=10.5,5.6 \mathrm{~Hz}, 2 \mathrm{H}), 7.04(\mathrm{~d}, J=8.3 \mathrm{~Hz}, 1 \mathrm{H}), 6.59(\mathrm{~d}, J=$ $3.2 \mathrm{~Hz}, 1 \mathrm{H}), 6.52(\mathrm{~d}, J=7.6 \mathrm{~Hz}, 1 \mathrm{H}), 4.80-4.72(\mathrm{~m}, 1 \mathrm{H}), 3.96(\mathrm{~s}, 3 \mathrm{H}), 2.25-2.16(\mathrm{~m}, 2 \mathrm{H}), 1.97-$ 
$1.85(\mathrm{~m}, 4 \mathrm{H}), 1.79-1.73(\mathrm{~m}, 2 \mathrm{H})$.

${ }^{13} \mathrm{C}$ NMR $\left(101 \mathrm{MHz}, \mathrm{CDCl}_{3}\right) \delta 153.3,137.5,123.0,121.9,119.0,103.3,99.0,98.1,57.1,55.3,32.6$, 24.0 .

IR (KBr) : 2957, 2873, 1580, 1492, 1254, 1064, $732 \mathrm{~cm}^{-1}$.

HRMS (ESI): Calcd for $\mathrm{C}_{14} \mathrm{H}_{18} \mathrm{NO}(\mathrm{M}+\mathrm{H})^{+}: 216.1383$; Found: 216.1387.

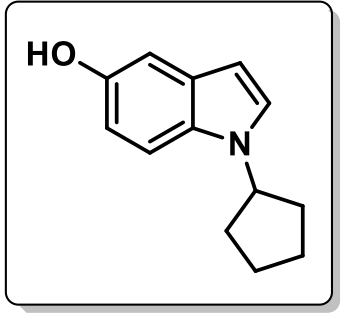

\section{1-cyclopentylindol-5-ol (3i)}

Colorless oil; $37.5 \mathrm{mg}, 93 \%$ yield.

${ }^{1} \mathbf{H}$ NMR $\left(400 \mathrm{MHz} \mathrm{CDCl}_{3}\right) \delta 7.24(\mathrm{~d}, J=8.8 \mathrm{~Hz}, 1 \mathrm{H}), 7.16(\mathrm{~d}, J=3.1 \mathrm{~Hz}, 1 \mathrm{H}), 7.03-7.00(\mathrm{~m}, 1 \mathrm{H})$, $6.77(\mathrm{~d}, J=8.8 \mathrm{~Hz}, 1 \mathrm{H}), 6.35(\mathrm{~d}, J=3.1 \mathrm{~Hz}, 1 \mathrm{H}), 4.94(\mathrm{~s}, 1 \mathrm{H}), 4.75-4.67(\mathrm{~m}, 1 \mathrm{H}), 2.22-2.13(\mathrm{~m}$, $2 \mathrm{H}), 1.95-1.83(\mathrm{~m}, 4 \mathrm{H}), 1.79-1.71(\mathrm{~m}, 2 \mathrm{H})$.

${ }^{13}$ C NMR (101 MHz, $\left.\mathrm{CDCl}_{3}\right) \delta$ 149.1, 131.6, 129.1, 125.4, 110.9, 110.4, 105.1, 99.9, 57.0, 32.5, 24.0.

IR (KBr) : 2959, 2873, 1481, 1457, 1237, 1148, 795, $715 \mathrm{~cm}^{-1}$.

HRMS (ESI): Calcd for $\mathrm{C}_{13} \mathrm{H}_{16} \mathrm{NO}(\mathrm{M}+\mathrm{H})^{+}$: 202.1226; Found: 202.1231.

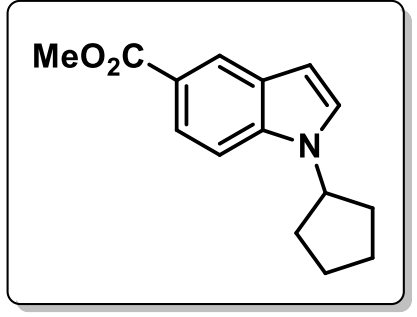

\section{methyl 1-cyclopentylindole-5-carboxylate (3j)}

Colorless oil; $45.6 \mathrm{mg}, 94 \%$ yield.

${ }^{1} \mathbf{H}$ NMR $\left(400 \mathrm{MHz}, \mathrm{CDCl}_{3}\right) \delta 8.39(\mathrm{~d}, J=1.3 \mathrm{~Hz}, 1 \mathrm{H}), 7.90(\mathrm{dd}, J=8.7,1.6 \mathrm{~Hz}, 1 \mathrm{H}), 7.39(\mathrm{~d}, J=8.8$ $\mathrm{Hz}, 1 \mathrm{H}), 7.24$ (d, J=3.3 Hz, 1H), $6.60-6.56(\mathrm{~m}, 1 \mathrm{H}), 4.83-4.74(\mathrm{~m}, 1 \mathrm{H}), 3.92$ (s, 3H), $2.25-2.15$ (m, 2H), $9.94-1.85(\mathrm{~m}, 4 \mathrm{H}), 1.81-1.70(\mathrm{~m}, 2 \mathrm{H})$.

${ }^{13} \mathbf{C}$ NMR $\left(101 \mathrm{MHz}, \mathrm{CDCl}_{3}\right) \delta 168.2,138.5,128.0,125.9,123.8,122.5,121.1,109.3,102.6,57.0$, $51.8,32.6,24.0$.

IR (KBr) : 2950, 2875, 1709, 1610, 1433, 1287, 1258, 1194, $751 \mathrm{~cm}^{-1}$.

HRMS (ESI): Calcd for $\mathrm{C}_{15} \mathrm{H}_{18} \mathrm{NO}_{2}(\mathrm{M}+\mathrm{H})^{+}:$244.1332; Found: 244.1338. 


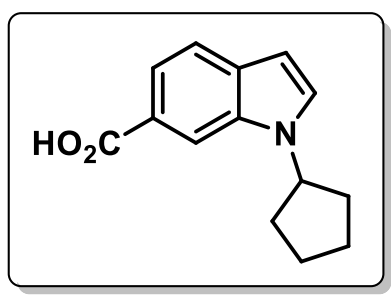

\section{1-cyclopentylindole-6-carboxylic acid (3k)}

white solid, Mp $159-160{ }^{\circ} \mathrm{C} ; 31.2 \mathrm{mg}, 68 \%$ yield.

${ }^{1} \mathbf{H}$ NMR $\left(400 \mathrm{MHz}, \mathrm{CDCl}_{3}\right) \delta 8.29(\mathrm{~s}, 1 \mathrm{H}), 7.88(\mathrm{dd}, J=8.3,1.4 \mathrm{~Hz}, 1 \mathrm{H}), 7.67(\mathrm{~d}, J=8.3 \mathrm{~Hz}, 1 \mathrm{H})$, $7.40(\mathrm{~d}, J=3.2 \mathrm{~Hz}, 1 \mathrm{H}), 6.56(\mathrm{~d}, J=3.0 \mathrm{~Hz}, 1 \mathrm{H}), 4.96-4.85(\mathrm{~m}, 1 \mathrm{H}), 2.32-2.22(\mathrm{~m}, 2 \mathrm{H}), 2.00-1.87$ (m, 4H), $1.85-1.75(\mathrm{~m}, 2 \mathrm{H})$.

${ }^{13}$ C NMR $\left(101 \mathrm{MHz}, \mathrm{CDCl}_{3}\right) \delta 173.7,135.4,132.9,128.3,121.7,120.8,120.4,113.0,101.6,57.1$, 32.8, 24.0.

IR (KBr) : 2987, 2875, 1677, 1610, 1459, 1412, 1263, $777 \mathrm{~cm}^{-1}$.

HRMS (ESI): Calcd for $\mathrm{C}_{14} \mathrm{H}_{16} \mathrm{NO}_{2}(\mathrm{M}+\mathrm{H})^{+}: 230.1176$; Found: 230.1182 .

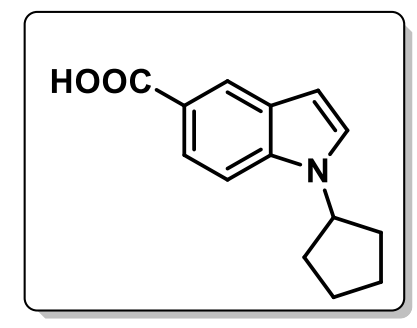

1-cyclopentylindole-5-carboxylic acid (3I)

white solid, Mp $170-171^{\circ} \mathrm{C} ; 32.8 \mathrm{mg}, 72 \%$ yield.

${ }^{1} \mathbf{H}$ NMR $\left(600 \mathrm{MHz}, \mathrm{CDCl}_{3}\right) \delta 8.49(\mathrm{~d}, J=1.3 \mathrm{~Hz}, 1 \mathrm{H}), 7.98(\mathrm{dd}, J=8.7,1.5 \mathrm{~Hz}, 1 \mathrm{H}), 7.43(\mathrm{~d}, J=8.7$ $\mathrm{Hz}, 1 \mathrm{H}), 7.28(\mathrm{~d}, J=3.2 \mathrm{~Hz}, 1 \mathrm{H}), 6.63(\mathrm{~d}, J=3.2 \mathrm{~Hz}, 1 \mathrm{H}), 4.83(\mathrm{dd}, J=14.2,6.9 \mathrm{~Hz}, 1 \mathrm{H}), 2.25(\mathrm{dt}, J=$ 13.6, 6.9 Hz, 2H), 1.93 (ddd, $J=13.9,10.6,5.4 \mathrm{~Hz}, 4 \mathrm{H}), 1.84-1.76(\mathrm{~m}, 2 \mathrm{H})$.

${ }^{13} \mathbf{C}$ NMR $\left(151 \mathrm{MHz}, \mathrm{CDCl}_{3}\right) \delta 173.2,139.1,128.3,126.1,124.9,123.1,120.4,109.5,103.0,57.3$, 32.7, 24.1.

IR (KBr) : 2963, 2874, 1674, 1608, 1417, 1311, $774 \mathrm{~cm}^{-1}$.

HRMS (ESI): Calcd for $\mathrm{C}_{14} \mathrm{H}_{16} \mathrm{NO}_{2}(\mathrm{M}+\mathrm{H})^{+}: 230.1176$; Found: 230.1181 .

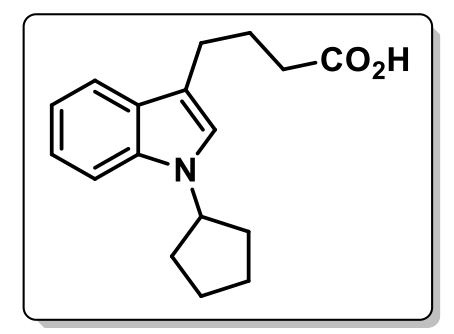

4-(1-cyclopentylindol-3-yl)butanoic acid (3 m)

Colorless oil; $43.5 \mathrm{mg}, 80 \%$ yield.

${ }^{1} \mathbf{H}$ NMR $\left(400 \mathrm{MHz}, \mathrm{CDCl}_{3}\right) \delta 7.64(\mathrm{~d}, J=7.8 \mathrm{~Hz}, 1 \mathrm{H}), 7.42(\mathrm{~d}, J=8.2 \mathrm{~Hz}, 1 \mathrm{H}), 7.25(\mathrm{t}, J=7.3 \mathrm{~Hz}$, $1 \mathrm{H}), 7.15(\mathrm{t}, J=7.2 \mathrm{~Hz}, 1 \mathrm{H}), 7.05(\mathrm{~s}, 1 \mathrm{H}), 4.85-4.76(\mathrm{~m}, 1 \mathrm{H}), 2.88(\mathrm{t}, J=7.4 \mathrm{~Hz}, 2 \mathrm{H}), 2.49(\mathrm{t}, J=7.4$ $\mathrm{Hz}, 2 \mathrm{H}), 2.28-2.21(\mathrm{~m}, 2 \mathrm{H}), 2.16-2.05(\mathrm{~m}, 2 \mathrm{H}), 2.01-1.90(\mathrm{~m}, 4 \mathrm{H}), 1.84-1.79(\mathrm{~m}, 2 \mathrm{H})$. 
${ }^{13}$ C NMR $\left(101 \mathrm{MHz}, \mathrm{CDCl}_{3}\right) \delta 180.2,136.5,127.9,122.0,121.2,118.9,118.6,113.8,109.7,56.7,33.7$, $32.6,25.2,24.5,24.1$.

IR (KBr) : 2955, 2871, 1707, 1463, 1231, $738 \mathrm{~cm}^{-1}$.

HRMS (ESI): Calcd for $\mathrm{C}_{17} \mathrm{H}_{22} \mathrm{NO}_{2}(\mathrm{M}+\mathrm{H})^{+}: 272.1645$; Found: 272.1650.

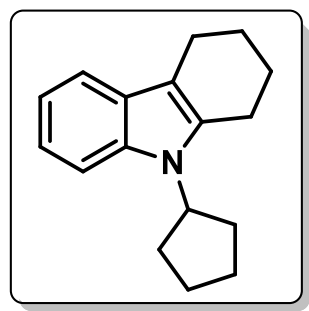

9-cyclopentyl-2,3,4,9-tetrahydrocarbazole (3n)

Colorless oil; $31.6 \mathrm{mg}, 66 \%$ yield.

${ }^{1} \mathbf{H}$ NMR $\left(600 \mathrm{MHz} \mathrm{CDCl}_{3}\right) \delta 7.48(\mathrm{~d}, J=7.6 \mathrm{~Hz}, 1 \mathrm{H}), 7.36(\mathrm{~d}, J=8.1 \mathrm{~Hz}, 1 \mathrm{H}), 7.09(\mathrm{dd}, J=11.1,4.0$ $\mathrm{Hz}, 1 \mathrm{H}), 7.05$ (t, $J=7.1 \mathrm{~Hz}, 1 \mathrm{H}), 4.70(\mathrm{p}, J=8.9 \mathrm{~Hz}, 1 \mathrm{H}), 2.76-2.71(\mathrm{~m}, 4 \mathrm{H}), 2.25-2.18(\mathrm{~m}, 2 \mathrm{H})$, $2.03-1.97(\mathrm{~m}, 4 \mathrm{H}), 1.96-1.92(\mathrm{~m}, 2 \mathrm{H}), 1.87-1.83(\mathrm{~m}, 2 \mathrm{H}), 1.78-1.72(\mathrm{~m}, 2 \mathrm{H})$.

${ }^{13} \mathbf{C}$ NMR $\left(151 \mathrm{MHz}, \mathrm{CDCl}_{3}\right) \delta 135.5,134.3,128.1,120.1,118.3,118.0,110.5,109.2,55.7,30.3,25.2$, 23.6, 23.2, 23.1, 21.1.

IR (KBr) : 2932, 2872, 1611, 1462, 1370, 1175, $736 \mathrm{~cm}^{-1}$.

HRMS (ESI): Calcd for $\mathrm{C}_{17} \mathrm{H}_{22} \mathrm{~N}(\mathrm{M}+\mathrm{H})^{+}:$240.1747; Found: 240.1752 .

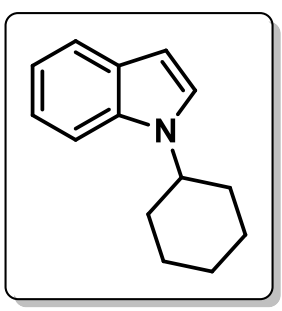

\section{1-cyclohexylindole (3o)}

Colorless oil; $35.9 \mathrm{mg}, 90 \%$ yield.

${ }^{1} \mathbf{H}$ NMR $\left(400 \mathrm{MHz}, \mathrm{CDCl}_{3}\right) \delta 7.63(\mathrm{~d}, J=7.9 \mathrm{~Hz}, 1 \mathrm{H}), 7.38(\mathrm{~d}, J=8.2 \mathrm{~Hz}, 1 \mathrm{H}), 7.24-7.21(\mathrm{~m}, 1 \mathrm{H})$, $7.21-7.16(\mathrm{~m}, 1 \mathrm{H}), 7.12-7.05(\mathrm{~m}, 1 \mathrm{H}), 6.50(\mathrm{~d}, J=3.1 \mathrm{~Hz}, 1 \mathrm{H}), 4.21(\mathrm{tt}, J=11.9,3.7 \mathrm{~Hz}, 1 \mathrm{H}), 2.13$ $(\mathrm{dd}, J=13.1,1.8 \mathrm{~Hz}, 2 \mathrm{H}), 1.96-1.89(\mathrm{~m}, 2 \mathrm{H}), 1.82-1.76(\mathrm{~m}, 1 \mathrm{H}), 1.70(\mathrm{ddd}, J=24.6,12.5,3.3 \mathrm{~Hz}$, $2 \mathrm{H}), 1.55-1.43(\mathrm{~m}, 2 \mathrm{H}), 1.34-1.23(\mathrm{~m}, 1 \mathrm{H})$.

${ }^{13} \mathrm{C}$ NMR (101 MHz, $\left.\mathrm{CDCl}_{3}\right) \delta 135.4,128.3,124.0,120.9$ (2C), 119.1, 109.4, 100.9, 55.0, 33.5, 25.9, 25.6 .

IR (KBr) : 2935, 2855, 1461, 1313, 1213, $736 \mathrm{~cm}^{-1}$.

HRMS (ESI): Calcd for $\mathrm{C}_{14} \mathrm{H}_{18} \mathrm{~N}(\mathrm{M}+\mathrm{H})^{+}:$: 200.1434; Found: 200.1433.

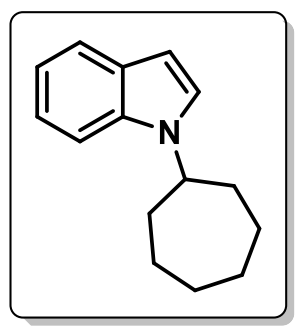




\section{1-cycloheptylindole (3p)}

Colorless oil; $31.1 \mathrm{mg}, 73 \%$ yield.

${ }^{1} \mathbf{H}$ NMR $\left(400 \mathrm{MHz}, \mathrm{CDCl}_{3}\right) \delta 7.63(\mathrm{~d}, J=7.9 \mathrm{~Hz}, 1 \mathrm{H}), 7.37(\mathrm{~d}, J=8.2 \mathrm{~Hz}, 1 \mathrm{H}), 7.20(\mathrm{ddd}, J=9.2$, $8.2,3.0 \mathrm{~Hz}, 2 \mathrm{H}), 7.11-7.06(\mathrm{~m}, 1 \mathrm{H}), 6.50(\mathrm{~d}, J=3.2 \mathrm{~Hz}, 1 \mathrm{H}), 4.41(\mathrm{tt}, J=10.3,4.1 \mathrm{~Hz}, 1 \mathrm{H}), 2.19-$ $2.11(\mathrm{~m}, 2 \mathrm{H}), 1.99-1.90(\mathrm{~m}, 2 \mathrm{H}), 1.87-1.79(\mathrm{~m}, 2 \mathrm{H}), 1.78-1.70(\mathrm{~m}, 2 \mathrm{H}), 1.69-1.57(\mathrm{~m}, 4 \mathrm{H})$.

${ }^{13} \mathrm{C}$ NMR (101 MHz, $\left.\mathrm{CDCl}_{3}\right) \delta 135.2,128.3,124.4,120.9$ (2C), 119.1, 109.5, 100.9, 57.3, 35.4, 27.7, 25.0 .

IR (KBr) : 2929, 2857, 1461, 1310, 1218, $736 \mathrm{~cm}^{-1}$.

HRMS (ESI): Calcd for $\mathrm{C}_{15} \mathrm{H}_{20} \mathrm{~N}(\mathrm{M}+\mathrm{H})^{+}: 214.1590$; Found: 214.1594 .

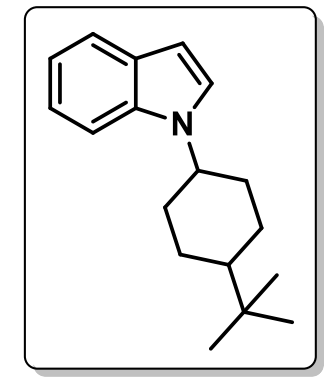

$95 \%$ yield.

1-(4-(tert-butyl)cyclohexyl)indole (3q) (major product)

Colorless oil. $32.3 \mathrm{mg}, 63 \%$ yield.

${ }^{1} \mathbf{H}$ NMR $\left(400 \mathrm{MHz}, \mathrm{CDCl}_{3}\right) \delta 7.64(\mathrm{~d}, J=7.9 \mathrm{~Hz}, 1 \mathrm{H}), 7.46(\mathrm{~d}, J=3.2 \mathrm{~Hz}, 1 \mathrm{H}), 7.35(\mathrm{~d}, J=8.3 \mathrm{~Hz}$, $1 \mathrm{H}), 7.21-7.16(\mathrm{~m}, 1 \mathrm{H}), 7.13-7.07(\mathrm{~m}, 1 \mathrm{H}), 6.49(\mathrm{~d}, J=3.2 \mathrm{~Hz}, 1 \mathrm{H}), 4.65-4.60(\mathrm{~m}, 1 \mathrm{H}), 2.35(\mathrm{~d}, J$ $=14.6 \mathrm{~Hz}, 2 \mathrm{H}), 1.91(\mathrm{tt}, J=14.2,4.6 \mathrm{~Hz}, 2 \mathrm{H}), 1.71(\mathrm{dd}, J=13.4,2.6 \mathrm{~Hz}, 2 \mathrm{H}), 1.38-1.27(\mathrm{~m}, 2 \mathrm{H})$, $1.22-1.16(\mathrm{~m}, 1 \mathrm{H}), 0.87(\mathrm{~s}, 9 \mathrm{H})$.

${ }^{13} \mathrm{C}$ NMR $\left(101 \mathrm{MHz}, \mathrm{CDCl}_{3}\right) \delta 136.0,128.3,126.3,121.0,120.8,119.2,110.1,100.3,49.7,47.3,32.6$, $30.5,27.4,22.9$.

IR (KBr) : 2937, 2862, 1463, 1364, 1217, $738 \mathrm{~cm}^{-1}$.

HRMS (ESI): Calcd for $\mathrm{C}_{18} \mathrm{H}_{26} \mathrm{~N}(\mathrm{M}+\mathrm{H})^{+}:$256.2060; Found: 256.2061.

\section{1-(4-(tert-butyl)cyclohexyl)indole (3q) (minor product)}

Colorless oil. $16.2 \mathrm{mg}, 32 \%$.

${ }^{1} \mathbf{H}$ NMR $\left(400 \mathrm{MHz} \mathrm{CDCl}_{3}\right) \delta 7.63(\mathrm{~d}, J=7.9 \mathrm{~Hz}, 1 \mathrm{H}), 7.39(\mathrm{~d}, J=8.2 \mathrm{~Hz}, 1 \mathrm{H}), 7.25-7.15(\mathrm{~m}, 2 \mathrm{H})$, 7.09 (t, $J=7.4 \mathrm{~Hz}, 1 \mathrm{H}), 6.51$ (d, $J=3.2 \mathrm{~Hz}, 1 \mathrm{H}), 4.19$ (tt, $J=12.0,3.8 \mathrm{~Hz}, 1 \mathrm{H}), 2.19(\mathrm{~d}, J=12.2 \mathrm{~Hz}$, 2H), 1.97 (d, $J=13.0 \mathrm{~Hz}, 2 \mathrm{H}), 1.73(\mathrm{qd}, J=12.5,2.9 \mathrm{~Hz}, 2 \mathrm{H}), 1.33-1.24(\mathrm{~m}, 2 \mathrm{H}), 1.20-1.12(\mathrm{~m}$, $1 \mathrm{H}), 0.92(\mathrm{~s}, 9 \mathrm{H})$.

${ }^{13}$ C NMR $\left(101 \mathrm{MHz}, \mathrm{CDCl}_{3}\right) \delta 135.5,128.3,123.9,121.0,120.9,119.1,109.4,100.8,55.1,47.4,33.5$, 32.4, 27.6, 26.7.

IR (KBr) : 2950, 2862, 1463, 1366, 1310, 1205, $734 \mathrm{~cm}^{-1}$. 


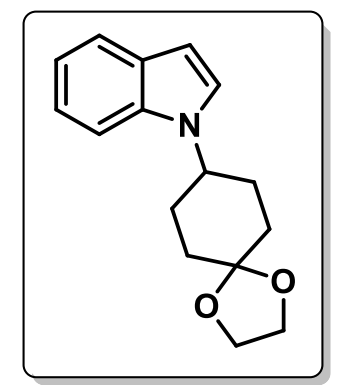

1-(1,4-dioxaspiro[4.5]decan-8-yl)indole (3r)

white solid, Mp $93-94{ }^{\circ} \mathrm{C} ; 47.2 \mathrm{mg}, 92 \%$ yield.

${ }^{1} \mathbf{H}$ NMR $\left(400 \mathrm{MHz}, \mathrm{CDCl}_{3}\right) \delta 7.62(\mathrm{~d}, J=7.9 \mathrm{~Hz}, 1 \mathrm{H}), 7.38(\mathrm{~d}, J=8.2 \mathrm{~Hz}, 1 \mathrm{H}), 7.23(\mathrm{~d}, J=3.3 \mathrm{~Hz}$, 1H), $7.22-7.17(\mathrm{~m}, 1 \mathrm{H}), 7.12-7.06(\mathrm{~m}, 1 \mathrm{H}), 6.50(\mathrm{~d}, J=3.1 \mathrm{~Hz}, 1 \mathrm{H}), 4.30(\mathrm{dt}, J=10.2,7.9 \mathrm{~Hz}, 1 \mathrm{H})$, 4.00 (s, 4H), $2.15-2.07$ (m, 4H), $1.95-1.89$ (m, 2H), $1.85-1.77(\mathrm{~m}, 2 \mathrm{H})$.

${ }^{13} \mathrm{C}$ NMR $\left(101 \mathrm{MHz}, \mathrm{CDCl}_{3}\right) \delta 135.6,128.5,124.1,121.2,121.0,119.3,109.3,107.7,101.2,64.5$, 64.4, 53.8, 34.1, 30.1.

IR (KBr) : 2948, 2883, 1463, 1312, 1217, 1107, 1036, 926, $739 \mathrm{~cm}^{-1}$.

HRMS (ESI): Calcd for $\mathrm{C}_{16} \mathrm{H}_{20} \mathrm{NO}_{2}(\mathrm{M}+\mathrm{H})^{+}: 258.1489$; Found: 258.1494.

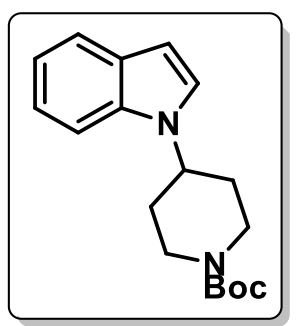

tert-butyl 4-(indol-1-yl)piperidine-1-carboxylate (3s)

Colorless oil; $54.7 \mathrm{mg}, 91 \%$ yield.

${ }^{1} \mathbf{H}$ NMR $\left(400 \mathrm{MHz} \mathrm{CDCl}_{3}\right) \delta 7.63(\mathrm{~d}, J=7.8 \mathrm{~Hz}, 1 \mathrm{H}), 7.36(\mathrm{~d}, J=8.2 \mathrm{~Hz}, 1 \mathrm{H}), 7.23-7.18(\mathrm{~m}, 1 \mathrm{H})$, $7.16(\mathrm{~d}, J=3.3 \mathrm{~Hz}, 1 \mathrm{H}), 7.13-7.08(\mathrm{~m}, 1 \mathrm{H}), 6.52(\mathrm{~d}, J=3.1 \mathrm{~Hz}, 1 \mathrm{H}), 4.39-4.30(\mathrm{~m}, 3 \mathrm{H}), 2.90(\mathrm{t}, J=$ $12.3 \mathrm{~Hz}, 2 \mathrm{H}), 2.06$ (d, $J=12.2 \mathrm{~Hz}, 2 \mathrm{H}), 1.94-1.84$ (m, 2H), 1.49 (s, 9H).

${ }^{13} \mathrm{C}$ NMR $\left(101 \mathrm{MHz}, \mathrm{CDCl}_{3}\right) \delta 154.6,135.4,128.5,123.7,121.4,121.1,119.5,109.1,101.7,99.9$, 79.9, 53.4, 32.3, 28.4 .

IR (KBr) : 2976, 2864, 1694, 1422, 1243, 1168, $739 \mathrm{~cm}^{-1}$.

HRMS (ESI): Calcd for $\mathrm{C}_{18} \mathrm{H}_{25} \mathrm{~N}_{2} \mathrm{O}_{2}(\mathrm{M}+\mathrm{H})^{+}: 301.1911$; Found: 301.1915 .

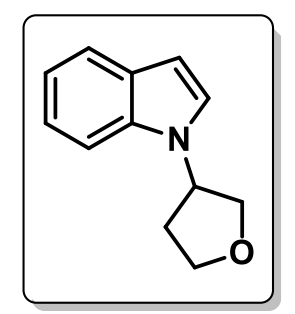

1-(tetrahydrofuran-3-yl)indole (3t)

Colorless oil; $35.1 \mathrm{mg}, 94 \%$ yield.

${ }^{1} \mathbf{H}$ NMR (400 MHz, $\left.\mathrm{CDCl}_{3}\right) \delta 7.63(\mathrm{~d}, J=7.9 \mathrm{~Hz}, 1 \mathrm{H}), 7.42-7.37(\mathrm{~m}, 1 \mathrm{H}), 7.26-7.19(\mathrm{~m}, 2 \mathrm{H}), 7.15$ 
$-7.09(\mathrm{~m}, 1 \mathrm{H}), 6.52(\mathrm{dd}, J=3.2,0.5 \mathrm{~Hz}, 1 \mathrm{H}), 5.06(\mathrm{ddd}, J=11.0,6.4,3.3 \mathrm{~Hz}, 1 \mathrm{H}), 4.13(\mathrm{td}, J=8.2$, $5.6 \mathrm{~Hz}, 2 \mathrm{H}), 4.05(\mathrm{dd}, J=9.8,6.0 \mathrm{~Hz}, 1 \mathrm{H}), 3.93(\mathrm{td}, J=8.6,6.1 \mathrm{~Hz}, 1 \mathrm{H}), 2.47(\mathrm{dtd}, J=13.4,8.1,6.6$ $\mathrm{Hz}, 1 \mathrm{H}), 2.22-2.13(\mathrm{~m}, 1 \mathrm{H})$.

${ }^{13} \mathbf{C}$ NMR $\left(101 \mathrm{MHz}, \mathrm{CDCl}_{3}\right) \delta 135.6,128.5,124.7,121.5,121.0,119.5,109.2,101.9,72.6,67.5,55.5$, 33.2 .

IR (KBr) : 2978, 2864, 1461, 1310, 1231, 1066, 915, $741 \mathrm{~cm}^{-1}$.

HRMS (ESI): Calcd for $\mathrm{C}_{12} \mathrm{H}_{14} \mathrm{NO}(\mathrm{M}+\mathrm{H})^{+}: 188.1070$; Found: 188.1073.

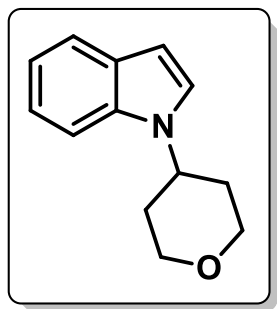

1-(tetrahydro-2H-pyran-4-yl)indole (3u)

Colorless oil; $33.3 \mathrm{mg}, 83 \%$ yield.

${ }^{1} \mathbf{H}$ NMR $\left(400 \mathrm{MHz}, \mathrm{CDCl}_{3}\right) \delta 7.64(\mathrm{~d}, J=7.8 \mathrm{~Hz}, 1 \mathrm{H}), 7.39(\mathrm{~d}, J=8.0 \mathrm{~Hz}, 1 \mathrm{H}), 7.24-7.19(\mathrm{~m}, 2 \mathrm{H})$, $7.15-7.09(\mathrm{~m}, 1 \mathrm{H}), 6.56-6.52(\mathrm{~m}, 1 \mathrm{H}), 4.50-4.41(\mathrm{~m}, 1 \mathrm{H}), 4.17-4.12(\mathrm{~m}, 2 \mathrm{H}), 3.61(\mathrm{dt}, J=14.3$, $7.2 \mathrm{~Hz}, 2 \mathrm{H}), 2.14-2.01(\mathrm{~m}, 4 \mathrm{H})$.

${ }^{13} \mathbf{C}$ NMR $\left(101 \mathrm{MHz}, \mathrm{CDCl}_{3}\right) \delta 135.3,128.5,123.8,121.3,121.1,119.5,109.1,101.6,67.4,52.2,33.3$. IR (KBr) : 2957, 2842, 1461, 1306, 1217, 1146, 1088, 1008, $739 \mathrm{~cm}^{-1}$.

HRMS (ESI): Calcd for $\mathrm{C}_{13} \mathrm{H}_{16} \mathrm{NO}(\mathrm{M}+\mathrm{H})^{+}$: 202.1226; Found: 202.1231.

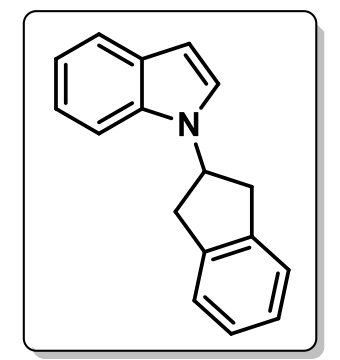

\section{1-(2,3-dihydro-1 $H$-inden-2-yl)indole (3v)}

white solid, $\mathrm{Mp} 101-102^{\circ} \mathrm{C} ; 30.4 \mathrm{mg}, 65 \%$ yield.

${ }^{1} \mathbf{H}$ NMR $\left(600 \mathrm{MHz}, \mathrm{CDCl}_{3}\right) \delta 7.63(\mathrm{~d}, J=7.9 \mathrm{~Hz}, 1 \mathrm{H}), 7.41(\mathrm{~d}, J=8.3 \mathrm{~Hz}, 1 \mathrm{H}), 7.29(\mathrm{dd}, J=5.0,3.3$ $\mathrm{Hz}, 2 \mathrm{H}), 7.25-7.23(\mathrm{~m}, 2 \mathrm{H}), 7.21(\mathrm{t}, J=7.7 \mathrm{~Hz}, 1 \mathrm{H}), 7.12(\mathrm{t}, J=7.5 \mathrm{~Hz}, 1 \mathrm{H}), 7.07(\mathrm{~d}, J=3.2 \mathrm{~Hz}, 1 \mathrm{H})$, $6.45(\mathrm{~d}, J=3.1 \mathrm{~Hz}, 1 \mathrm{H}), 5.37-5.31(\mathrm{~m}, 1 \mathrm{H}), 3.54(\mathrm{dd}, J=16.3,7.8 \mathrm{~Hz}, 2 \mathrm{H}), 3.32(\mathrm{dd}, J=16.2,5.5$ $\mathrm{Hz}, 2 \mathrm{H})$.

${ }^{13}$ C NMR $\left(151 \mathrm{MHz}, \mathrm{CDCl}_{3}\right) \delta 140.7,135.7,128.7,127.1,124.8,124.7,121.4,121.0,119.5,109.6$, $101.4,55.9,39.8$.

IR (KBr) : 3420, 3049, 2946, 1611, 1478, 1311, 1237, $740 \mathrm{~cm}^{-1}$.

HRMS (ESI): Calcd for $\mathrm{C}_{17} \mathrm{H}_{16} \mathrm{~N}(\mathrm{M}+\mathrm{H})^{+}:$234.1277; Found: 234.1285. 


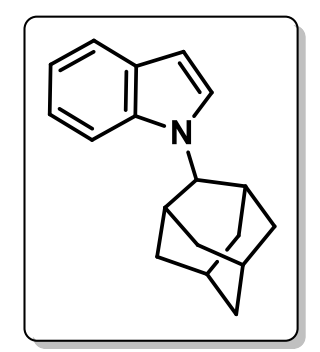

\section{1-((1r,3r)-adamantan-2-yl)indole (3w)}

white solid, Mp $99-100{ }^{\circ} \mathrm{C} ; 28.7 \mathrm{mg}, 57 \%$ yield.

${ }^{1} \mathbf{H}$ NMR $\left(400 \mathrm{MHz}, \mathrm{CDCl}_{3}\right) \delta 7.64(\mathrm{~d}, J=7.7 \mathrm{~Hz}, 1 \mathrm{H}), 7.56(\mathrm{~d}, J=3.3 \mathrm{~Hz}, 1 \mathrm{H}), 7.32(\mathrm{~d}, J=8.3 \mathrm{~Hz}$, $1 \mathrm{H}), 7.21-7.15(\mathrm{~m}, 1 \mathrm{H}), 7.13-7.07(\mathrm{~m}, 1 \mathrm{H}), 6.50(\mathrm{dd}, J=3.3,0.7 \mathrm{~Hz}, 1 \mathrm{H}), 4.54(\mathrm{~s}, 1 \mathrm{H}), 2.56(\mathrm{~s}, 2 \mathrm{H})$, $2.11-2.00(\mathrm{~m}, 7 \mathrm{H}), 1.97(\mathrm{~s}, 1 \mathrm{H}), 1.83(\mathrm{~s}, 2 \mathrm{H}), 1.73(\mathrm{~d}, J=12.4 \mathrm{~Hz}, 2 \mathrm{H})$.

${ }^{13} \mathbf{C ~ N M R}\left(101 \mathrm{MHz}, \mathrm{CDCl}_{3}\right) \delta 136.2,128.5,125.9,120.9,120.8,119.2,110.6,100.3,60.8,38.3,37.6$, $32.3,32.1,27.7,27.2$.

IR (KBr) : 2911, 2853, 1463, 1304, 1224, 739, $721 \mathrm{~cm}^{-1}$.

HRMS (ESI): Calcd for $\mathrm{C}_{18} \mathrm{H}_{22} \mathrm{~N}(\mathrm{M}+\mathrm{H})^{+}: 252.1747$; Found: 252.1752 .

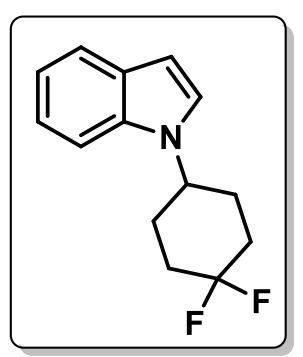

\section{1-(4,4-difluorocyclohexyl)indole (3x)}

Colorless oil; $36.7 \mathrm{mg}, 78 \%$ yield.

${ }^{1} \mathbf{H}$ NMR $\left(600 \mathrm{MHz}, \mathrm{CDCl}_{3}\right) \delta 7.65(\mathrm{~d}, J=7.9 \mathrm{~Hz}, 1 \mathrm{H}), 7.36(\mathrm{~d}, J=8.3 \mathrm{~Hz}, 1 \mathrm{H}), 7.24-7.21(\mathrm{~m}, 1 \mathrm{H})$, $7.19(\mathrm{~d}, J=3.2 \mathrm{~Hz}, 1 \mathrm{H}), 7.15-7.10(\mathrm{~m}, 1 \mathrm{H}), 6.54(\mathrm{~d}, J=3.2 \mathrm{~Hz}, 1 \mathrm{H}), 4.36(\mathrm{td}, J=11.8,5.7 \mathrm{~Hz}, 1 \mathrm{H})$, $2.33-2.28(\mathrm{~m}, 2 \mathrm{H}), 2.19-2.07(\mathrm{~m}, 4 \mathrm{H}), 2.06-1.94(\mathrm{~m}, 2 \mathrm{H})$.

${ }^{13} \mathbf{C}$ NMR (151 MHz, $\left.\mathrm{CDCl}_{3}\right) \delta 135.5,128.6,123.7,122.2(\mathrm{t}, J=243,4 \mathrm{~Hz}), 121.5,121.2,119.6,109.1$, $101.8,52.9,33.1(\mathrm{t}, J=24,9 \mathrm{~Hz}), 28.89(\mathrm{~d}, J=10.1 \mathrm{~Hz})$.

${ }^{19}$ F NMR $\left(376 \mathrm{MHz}, \mathrm{CDCl}_{3}\right) \delta-93.57--94.56(\mathrm{~m}, 1 \mathrm{~F}),-101.91--102.77(\mathrm{~m}, 1 \mathrm{~F})$.

IR (KBr) : 3423, 2946, 1613, 1477, 1313, 1218, 1110, 958, $742 \mathrm{~cm}^{-1}$.

HRMS (ESI): Calcd for $\mathrm{C}_{14} \mathrm{H}_{16} \mathrm{~F}_{2} \mathrm{~N}(\mathrm{M}+\mathrm{H})^{+}: 236.1245$; Found: 236.1253 .

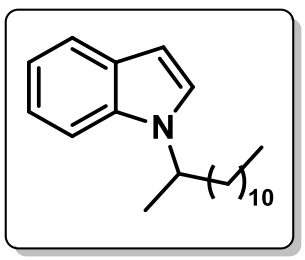

\section{1-(tridecan-2-yl)indole (3y)}

Colorless oil; $57.5 \mathrm{mg}, 96 \%$ yield.

${ }^{1} \mathbf{H}$ NMR $\left(400 \mathrm{MHz} \mathrm{CDCl}_{3}\right) \delta 7.63(\mathrm{~d}, J=7.8 \mathrm{~Hz}, 1 \mathrm{H}), 7.37(\mathrm{~d}, J=8.3 \mathrm{~Hz}, 1 \mathrm{H}), 7.22-7.16(\mathrm{~m}, 2 \mathrm{H})$, $7.09(\mathrm{t}, J=7.4 \mathrm{~Hz}, 1 \mathrm{H}), 6.52(\mathrm{~d}, J=3.2 \mathrm{~Hz}, 1 \mathrm{H}), 4.52-4.40(\mathrm{~m}, 1 \mathrm{H}), 1.96-1.84(\mathrm{~m}, 1 \mathrm{H}), 1.84-1.74$ 
$(\mathrm{m}, 1 \mathrm{H}), 1.49(\mathrm{~d}, J=6.8 \mathrm{~Hz}, 3 \mathrm{H}), 1.35-1.15(\mathrm{~m}, 19 \mathrm{H}), 0.87(\mathrm{t}, J=6.9 \mathrm{~Hz}, 3 \mathrm{H})$.

${ }^{13} \mathrm{C}$ NMR (101 MHz, $\left.\mathrm{CDCl}_{3}\right) \delta 135.8,128.3,124.0,120.9$ (2C), 119.1, 109.4, 101.2, 51.5, 37.1, 31.9, $29.68-29.23(\mathrm{~m}), 26.4,22.7,21.4,14.1$.

IR (KBr) : 2926, 2855, 1459, 1310, 1220, $738 \mathrm{~cm}^{-1}$.

HRMS (ESI): Calcd for $\mathrm{C}_{21} \mathrm{H}_{34} \mathrm{~N}(\mathrm{M}+\mathrm{H})^{+}:$300.2686; Found: 300.2689.

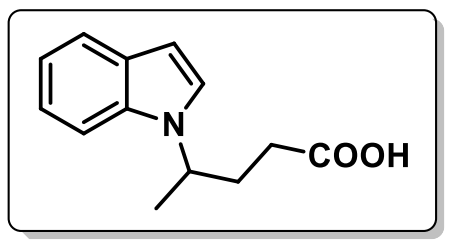

4-(indol-1-yl)pentanoic acid (3z)

Colorless oil; $22.2 \mathrm{mg}, 51 \%$ yield.

${ }^{1} \mathbf{H}$ NMR $\left(400 \mathrm{MHz} \mathrm{CDCl}_{3}\right) \delta 7.63(\mathrm{~d}, J=7.8 \mathrm{~Hz}, 1 \mathrm{H}), 7.36(\mathrm{~d}, J=8.2 \mathrm{~Hz}, 1 \mathrm{H}), 7.22-7.17(\mathrm{~m}, 1 \mathrm{H})$, $7.16(\mathrm{~d}, J=3.2 \mathrm{~Hz}, 1 \mathrm{H}), 7.12-7.08(\mathrm{~m}, 1 \mathrm{H}), 6.55(\mathrm{~d}, J=3.2 \mathrm{~Hz}, 1 \mathrm{H}), 4.63-4.56(\mathrm{~m}, 1 \mathrm{H}), 2.24-2.13$ $(\mathrm{m}, 4 \mathrm{H}), 1.54(\mathrm{~d}, J=6.8 \mathrm{~Hz}, 3 \mathrm{H})$.

${ }^{13} \mathbf{C}$ NMR $\left(101 \mathrm{MHz}, \mathrm{CDCl}_{3}\right) \delta 179.0,135.9,128.4,123.7,121.4,121.0,119.4,109.3,102.0,50.5$, $31.8,30.5,21.3$.

IR (KBr) : 2978, 2935, 1709, 1461, 1306, 1224, $741 \mathrm{~cm}^{-1}$.

HRMS (ESI): Calcd for $\mathrm{C}_{13} \mathrm{H}_{16} \mathrm{NO}_{2}(\mathrm{M}+\mathrm{H})^{+}: 218.1176$; Found: 218.1181 .

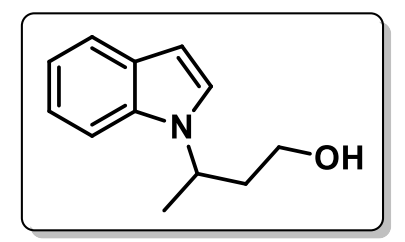

\section{3-(indol-1-yl)butan-1-ol (3aa)}

Colorless oil; $15.8 \mathrm{mg}, 42 \%$ yield.

${ }^{1} \mathbf{H}$ NMR $\left(400 \mathrm{MHz}, \mathrm{CDCl}_{3}\right) \delta 7.67(\mathrm{~d}, J=7.9 \mathrm{~Hz}, 1 \mathrm{H}), 7.47(\mathrm{~d}, J=8.2 \mathrm{~Hz}, 1 \mathrm{H}), 7.27-7.20(\mathrm{~m}, 2 \mathrm{H})$, $7.16-7.11(\mathrm{~m}, 1 \mathrm{H}), 6.59(\mathrm{~d}, J=3.1 \mathrm{~Hz}, 1 \mathrm{H}), 4.81(\mathrm{dq}, J=13.4,6.8 \mathrm{~Hz}, 1 \mathrm{H}), 3.58(\mathrm{dt}, J=10.7,5.4 \mathrm{~Hz}$, $1 \mathrm{H}), 3.36$ (ddd, $J=10.7,7.8,5.6 \mathrm{~Hz}, 1 \mathrm{H}), 2.16-2.05(\mathrm{~m}, 2 \mathrm{H}), 1.59$ (d, $J=6.9 \mathrm{~Hz}, 3 \mathrm{H}), 1.43(\mathrm{~s}, 1 \mathrm{H})$.

${ }^{13} \mathbf{C}$ NMR $\left(101 \mathrm{MHz}, \mathrm{CDCl}_{3}\right) \delta 135.9,128.2,123.9,121.3,120.9,119.3,109.4,101.7,59.3,47.8,39.5$, 21.4 .

IR (KBr) : 3395, 2974, 2933, 1461, 1306, 1228, 1049, $741 \mathrm{~cm}^{-1}$.

HRMS (ESI): Calcd for $\mathrm{C}_{12} \mathrm{H}_{16} \mathrm{NO}(\mathrm{M}+\mathrm{H})^{+}$: 190.1226; Found: 190.1230.

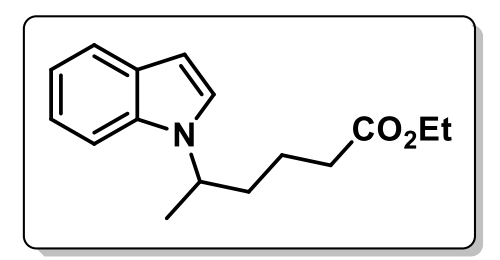

ethyl 5-(indol-1-yl)hexanoate (3ab)

Colorless oil; $41.5 \mathrm{mg}, 80 \%$ yield.

${ }^{1} \mathbf{H}$ NMR $\left(400 \mathrm{MHz}, \mathrm{CDCl}_{3}\right) \delta 7.63(\mathrm{~d}, J=7.9 \mathrm{~Hz}, 1 \mathrm{H}), 7.37(\mathrm{~d}, J=8.3 \mathrm{~Hz}, 1 \mathrm{H}), 7.19(\mathrm{dd}, J=8.4,5.6$ 
Hz, 2H), 7.09 (t, $J=7.4 \mathrm{~Hz}, 1 \mathrm{H}), 6.53(\mathrm{~d}, J=3.2 \mathrm{~Hz}, 1 \mathrm{H}), 4.55-4.45(\mathrm{~m}, 1 \mathrm{H}), 4.09$ (q, $J=7.1 \mathrm{~Hz}$, $2 \mathrm{H}), 2.25(\mathrm{t}, J=7.3 \mathrm{~Hz}, 2 \mathrm{H}), 1.98-1.81(\mathrm{~m}, 2 \mathrm{H}), 1.61-1.55(\mathrm{~m}, 1 \mathrm{H}), 1.51(\mathrm{~d}, J=6.8 \mathrm{~Hz}, 3 \mathrm{H}), 1.49-$ $1.41(\mathrm{~m}, 1 \mathrm{H}), 1.22(\mathrm{t}, J=7.1 \mathrm{~Hz}, 3 \mathrm{H})$.

${ }^{13} \mathbf{C}$ NMR $\left(101 \mathrm{MHz}, \mathrm{CDCl}_{3}\right) \delta 173.2,135.7,128.4,123.8,121.2,120.9,119.2,109.3,101.5,60.3$, 51.2, 36.3, 33.7, 21.7, 21.3, 14.2.

IR (KBr) : 2980, 1731, 1463, 1310, 1179, $739 \mathrm{~cm}^{-1}$.

HRMS (ESI): Calcd for $\mathrm{C}_{16} \mathrm{H}_{22} \mathrm{NO}_{2}(\mathrm{M}+\mathrm{H})^{+}: 260.1645$; Found: 260.1647.

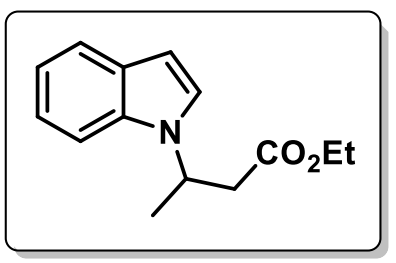

ethyl 3-(indol-1-yl)butanoate (3ac)

Colorless oil; $39.4 \mathrm{mg}, 85 \%$ yield.

${ }^{1}$ H NMR (400 MHz, $\left.\mathrm{CDCl}_{3}\right) \delta 7.62(\mathrm{~d}, J=7.9 \mathrm{~Hz}, 1 \mathrm{H}), 7.43(\mathrm{~d}, J=8.0 \mathrm{~Hz}, 1 \mathrm{H}), 7.23-7.19(\mathrm{~m}, 1 \mathrm{H})$, $7.18(\mathrm{~d}, J=3.3 \mathrm{~Hz}, 1 \mathrm{H}), 7.13-7.07(\mathrm{~m}, 1 \mathrm{H}), 6.53(\mathrm{~d}, J=3.2 \mathrm{~Hz}, 1 \mathrm{H}), 5.08-4.97$ (m, $1 \mathrm{H}), 4.08-3.98$ $(\mathrm{m}, 2 \mathrm{H}), 2.90(\mathrm{dd}, J=15.4,6.8 \mathrm{~Hz}, 1 \mathrm{H}), 2.76(\mathrm{dd}, J=15.4,7.5 \mathrm{~Hz}, 1 \mathrm{H}), 1.59(\mathrm{~d}, J=6.8 \mathrm{~Hz}, 3 \mathrm{H}), 1.12$ (t, $J=7.1 \mathrm{~Hz}, 3 \mathrm{H})$.

${ }^{13} \mathbf{C}$ NMR $\left(101 \mathrm{MHz}, \mathrm{CDCl}_{3}\right) \delta 170.7,135.4,128.4,123.8,121.4,120.9,119.4,109.5,101.9,60.7$, 48.2, 41.9, 20.8, 14.0 .

IR (KBr) : 2983, 2939, 1731, 1461, 1308, 1220, 1181, 1038, $739 \mathrm{~cm}^{-1}$.

HRMS (ESI): Calcd for $\mathrm{C}_{14} \mathrm{H}_{18} \mathrm{NO}_{2}(\mathrm{M}+\mathrm{H})^{+}: 232.1332$; Found: 232.1333 .

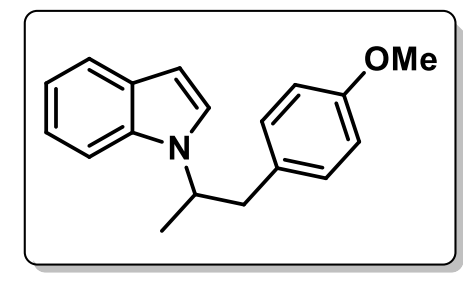

\section{1-(1-(4-methoxyphenyl)propan-2-yl)indole (3ad)}

Colorless oil; $40.4 \mathrm{mg}, 76 \%$ yield.

${ }^{1} \mathbf{H}$ NMR $\left(400 \mathrm{MHz}, \mathrm{CDCl}_{3}\right) \delta 7.63(\mathrm{~d}, J=7.8 \mathrm{~Hz}, 1 \mathrm{H}), 7.35(\mathrm{~d}, J=8.0 \mathrm{~Hz}, 1 \mathrm{H}), 7.21-7.15(\mathrm{~m}, 1 \mathrm{H})$, $7.12(\mathrm{~d}, J=3.2 \mathrm{~Hz}, 1 \mathrm{H}), 7.11-7.06(\mathrm{~m}, 1 \mathrm{H}), 6.94-6.88(\mathrm{~m}, 2 \mathrm{H}), 6.78-6.70(\mathrm{~m}, 2 \mathrm{H}), 6.50(\mathrm{~d}, J=2.8$ $\mathrm{Hz}, 1 \mathrm{H}), 4.71-4.61(\mathrm{~m}, 1 \mathrm{H}), 3.74(\mathrm{~s}, 3 \mathrm{H}), 3.03(\mathrm{ddd}, J=21.1,13.6,6.8 \mathrm{~Hz}, 2 \mathrm{H}), 1.50(\mathrm{~d}, J=6.8 \mathrm{~Hz}$, $3 \mathrm{H})$

${ }^{13}$ C NMR $\left(101 \mathrm{MHz}, \mathrm{CDCl}_{3}\right) \delta 158.2,135.5,130.1,130.0,128.5,124.2,121.1,120.9,119.2,113.7$, $109.5,101.1,55.2,53.0,42.3,19.9$.

IR (KBr) : 3045, 2978, 2838, 1612, 1513, 1463, 1306, 1248, 1179, 1034, 816, $739 \mathrm{~cm}^{-1}$.

HRMS (ESI): Calcd for $\mathrm{C}_{18} \mathrm{H}_{20} \mathrm{NO}(\mathrm{M}+\mathrm{H})^{+}$: 266.1539; Found: 266.1541. 


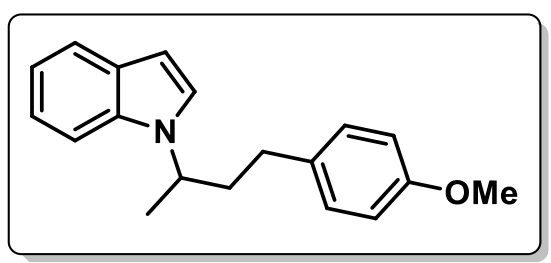

1-(4-(4-methoxyphenyl)butan-2-yl)indole (3ae)

Colorless oil; $47.5 \mathrm{mg}, 85 \%$ yield.

${ }^{1} \mathbf{H}$ NMR $\left(400 \mathrm{MHz}, \mathrm{CDCl}_{3}\right) \delta 7.64(\mathrm{~d}, J=7.8 \mathrm{~Hz}, 1 \mathrm{H}), 7.28(\mathrm{~d}, J=8.2 \mathrm{~Hz}, 1 \mathrm{H}), 7.22-7.16(\mathrm{~m}, 2 \mathrm{H})$, $7.13-7.07(\mathrm{~m}, 1 \mathrm{H}), 6.98(\mathrm{t}, J=5.7 \mathrm{~Hz}, 2 \mathrm{H}), 6.82-6.77(\mathrm{~m}, 2 \mathrm{H}), 6.56(\mathrm{~d}, J=3.2 \mathrm{~Hz}, 1 \mathrm{H}), 4.51-4.40$ $(\mathrm{m}, 1 \mathrm{H}), 3.77(\mathrm{~s}, 3 \mathrm{H}), 2.44(\mathrm{t}, J=7.7 \mathrm{~Hz}, 2 \mathrm{H}), 2.28-2.18(\mathrm{~m}, 1 \mathrm{H}), 2.08(\mathrm{dtd}, J=13.7,8.1,5.5 \mathrm{~Hz}$, $1 \mathrm{H}), 1.49(\mathrm{~d}, J=6.8 \mathrm{~Hz}, 3 \mathrm{H})$.

${ }^{13}$ C NMR $\left(101 \mathrm{MHz}, \mathrm{CDCl}_{3}\right) \delta 157.8,135.8,133.1,129.3,128.4,123.9,121.1,120.9,119.2,113.7$, $109.5,101.6,55.2,50.6,38.8,31.5,21.5$.

IR (KBr) : 3065, 2935, 1612, 1513, 1461, 1302, 1246, 1179, 1036, $741 \mathrm{~cm}^{-1}$.

HRMS (ESI): Calcd for $\mathrm{C}_{19} \mathrm{H}_{22} \mathrm{NO}(\mathrm{M}+\mathrm{H})^{+}: 280.1696$; Found: 280.1701 .

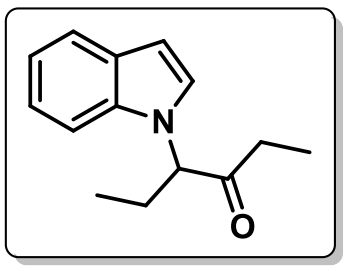

4-(indol-1-yl)hexan-3-one (3af)

Colorless oil; $27.4 \mathrm{mg}, 64 \%$ yield.

${ }^{1} \mathbf{H}$ NMR $\left(600 \mathrm{MHz}, \mathrm{CDCl}_{3}\right) \delta 7.66(\mathrm{~d}, J=7.8 \mathrm{~Hz}, 1 \mathrm{H}), 7.28(\mathrm{~d}, J=8.2 \mathrm{~Hz}, 1 \mathrm{H}), 7.22(\mathrm{t}, J=7.6 \mathrm{~Hz}$, $1 \mathrm{H}), 7.13-7.17(\mathrm{~m}, 2 \mathrm{H}), 6.61(\mathrm{~d}, J=3.1 \mathrm{~Hz}, 1 \mathrm{H}), 4.75(\mathrm{dd}, J=9.9,5.5 \mathrm{~Hz}, 1 \mathrm{H}), 2.31-2.13(\mathrm{~m}, 3 \mathrm{H})$, $2.12-2.06(\mathrm{~m}, 1 \mathrm{H}), 0.92(\mathrm{t}, J=7.2 \mathrm{~Hz}, 3 \mathrm{H}), 0.87(\mathrm{t}, J=7.4 \mathrm{~Hz}, 3 \mathrm{H})$.

${ }^{13}$ C NMR $\left(151 \mathrm{MHz}, \mathrm{CDCl}_{3}\right) \delta 208.8,136.4,128.7,125.5,122.1,121.2,119.9,109.1,102.9,65.9$, $32.2,23.4,10.7,7.4$

IR (KBr) : 2973, 2938, 1721, 1460, 1310, 1215, $742 \mathrm{~cm}^{-1}$.

HRMS (ESI): Calcd for $\mathrm{C}_{14} \mathrm{H}_{18} \mathrm{NO}(\mathrm{M}+\mathrm{H})^{+}: 216.1383$; Found: 216.1387. 


\section{Copies of ${ }^{1} \mathrm{H}$ NMR, ${ }^{19}$ F NMR and ${ }^{13} \mathrm{C}$ NMR}

${ }^{1} \mathrm{H}$ NMR spectrum of compound 3a $\left(\mathrm{CDCl}_{3}, 400 \mathrm{MHz}\right)$

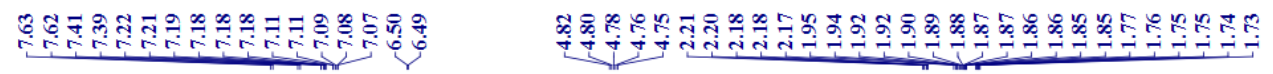

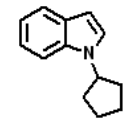

${ }^{1} \mathrm{H}$ NMR $\left(400 \mathrm{MHz}, \mathrm{CDCl}_{3}\right)$

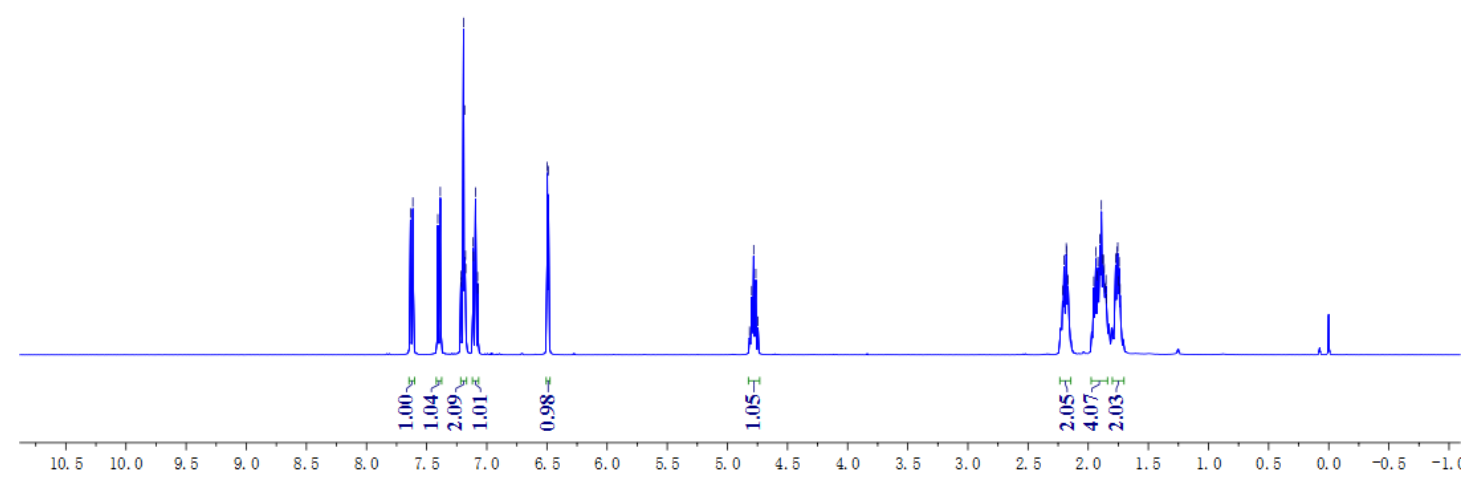

${ }^{13} \mathrm{C}$ NMR spectrum of compound 3a $\left(\mathrm{CDCl}_{3}, 101 \mathrm{MHz}\right)$
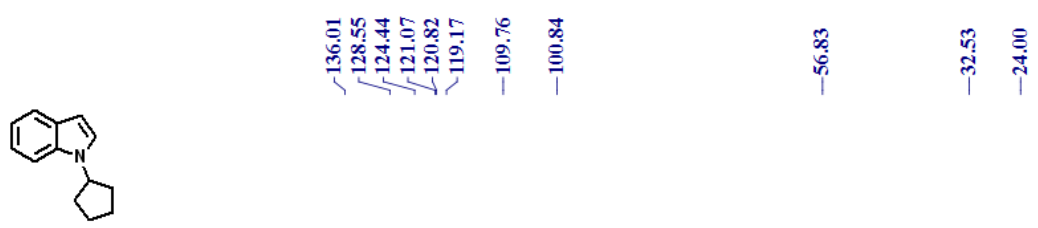

${ }^{13} \mathrm{C}$ NMR $(101 \mathrm{MHz}, \mathrm{CDCl})$

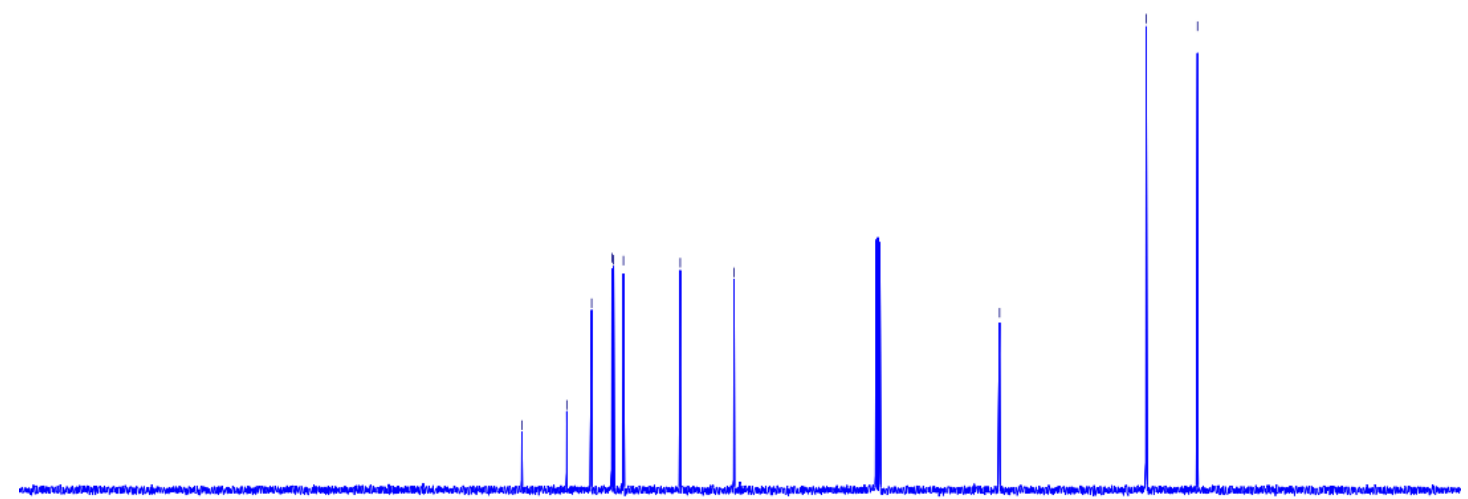

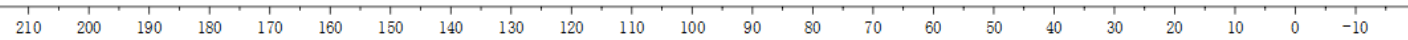


${ }^{1} \mathrm{H}$ NMR spectrum of compound $\mathbf{3 b}\left(\mathrm{CDCl}_{3}, 400 \mathrm{MHz}\right)$

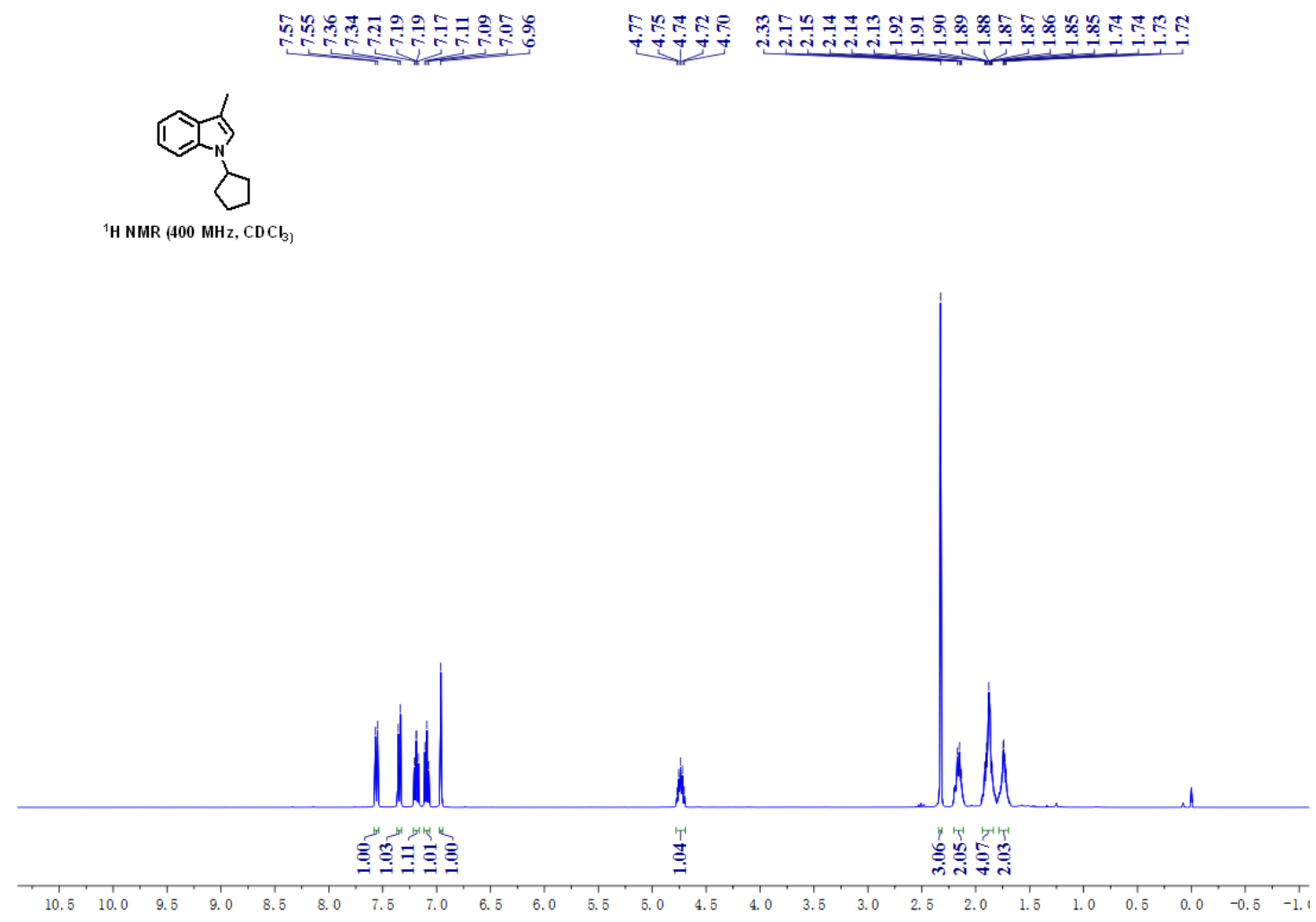

${ }^{13} \mathrm{C}$ NMR spectrum of compound $\mathbf{3 b}\left(\mathrm{CDCl}_{3}, 101 \mathrm{MHz}\right)$
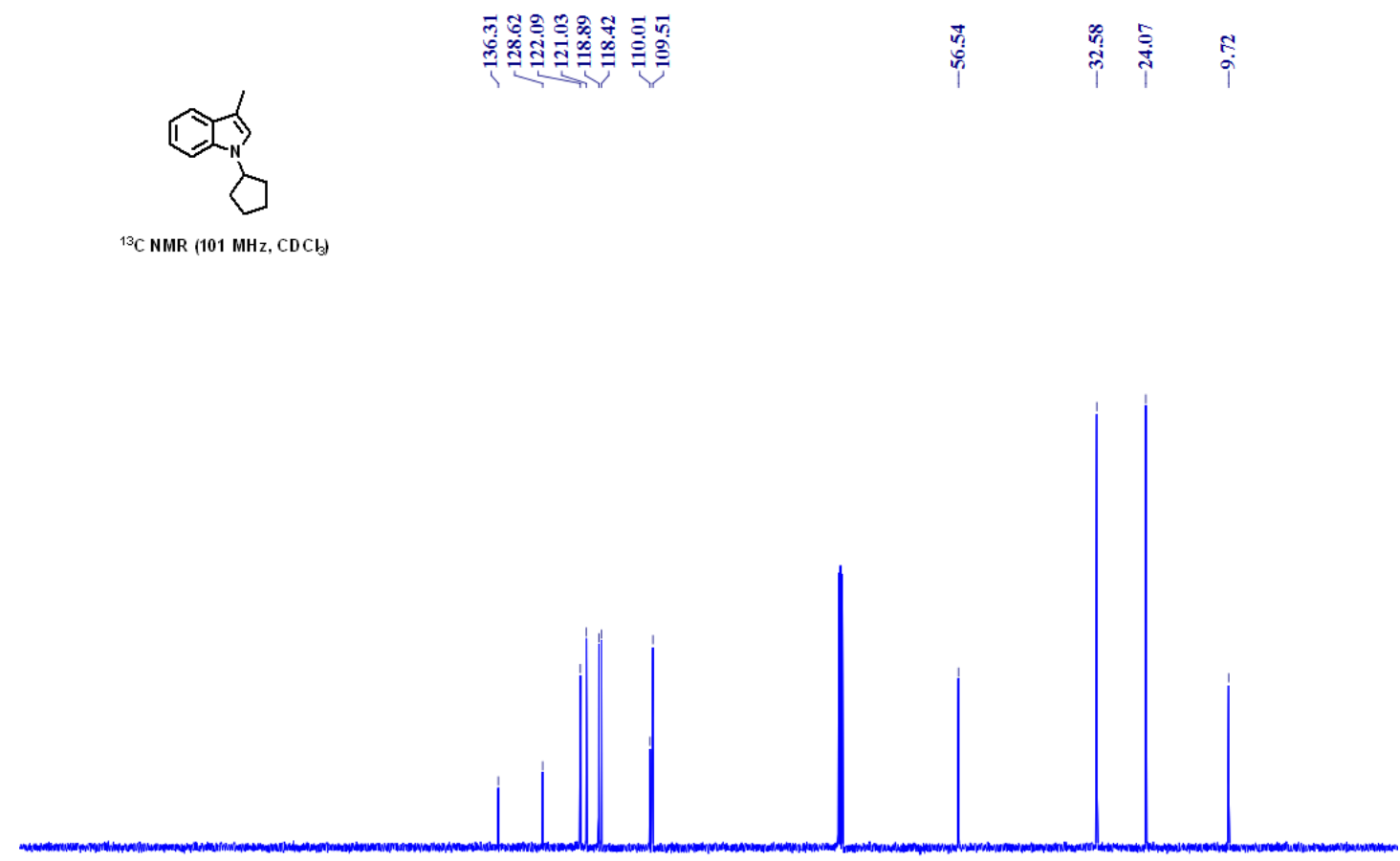

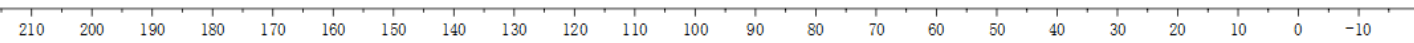


${ }^{1} \mathrm{H}$ NMR spectrum of compound $\mathbf{3 c}\left(\mathrm{CDCl}_{3}, 400 \mathrm{MHz}\right)$

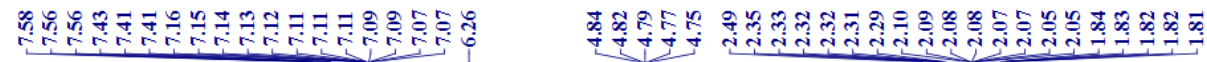

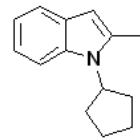

${ }^{1} \mathrm{H}$ NMR $\left(400 \mathrm{MHz}, \mathrm{CDCl}_{3}\right)$

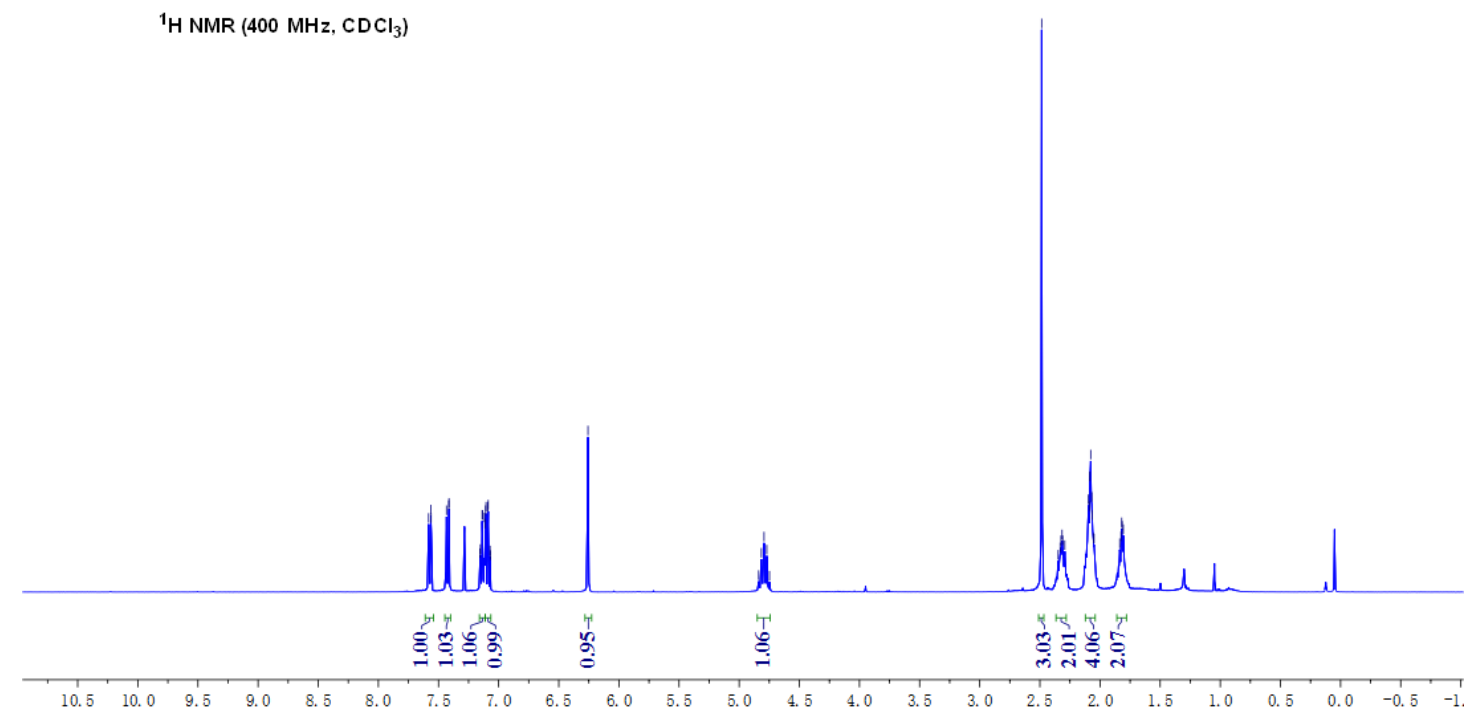

${ }^{13} \mathrm{C}$ NMR spectrum of compound $3 \mathbf{c}\left(\mathrm{CDCl}_{3}, 101 \mathrm{MHz}\right)$
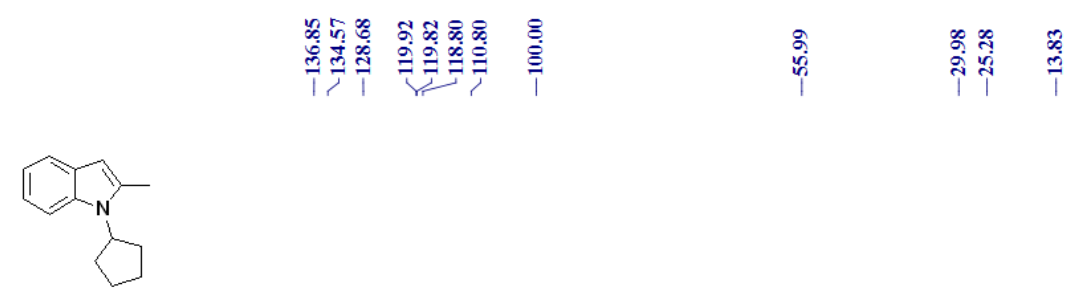

${ }^{13} \mathrm{C}$ NMR (101 MHz, CDCl 3$)$

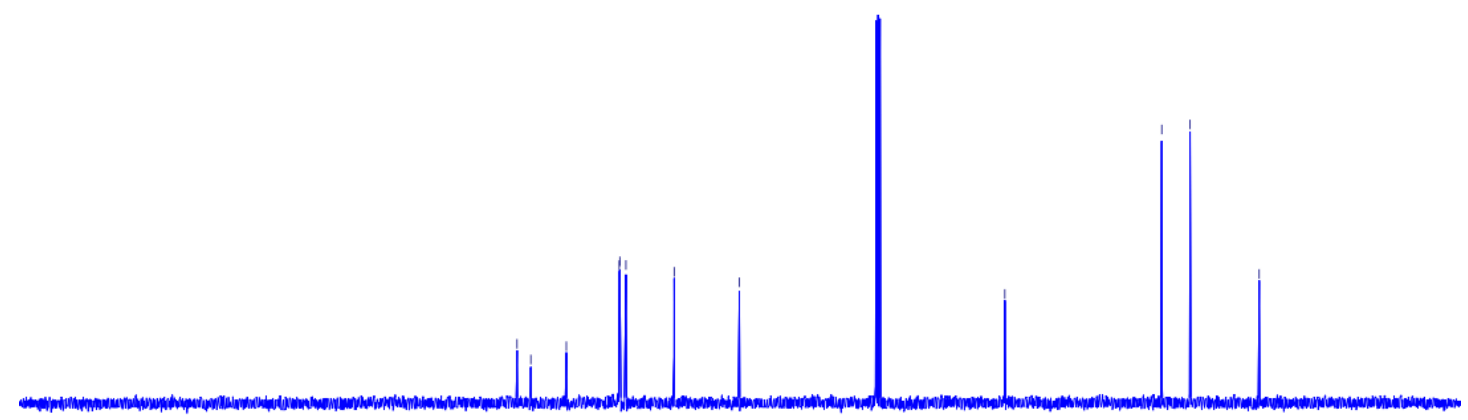

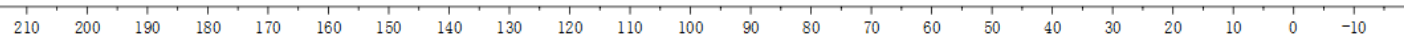


${ }^{1} \mathrm{H}$ NMR spectrum of compound $\mathbf{3 d}\left(\mathrm{CDCl}_{3}, 400 \mathrm{MHz}\right)$

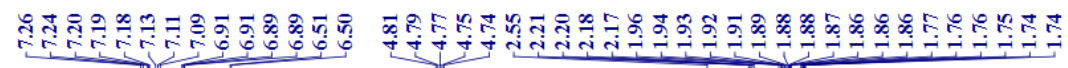

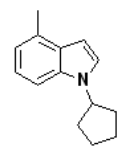

$\left.{ }^{1} \mathrm{H} \mathrm{NMR} \mathrm{(CDCl}, 400 \mathrm{MHz}\right)$

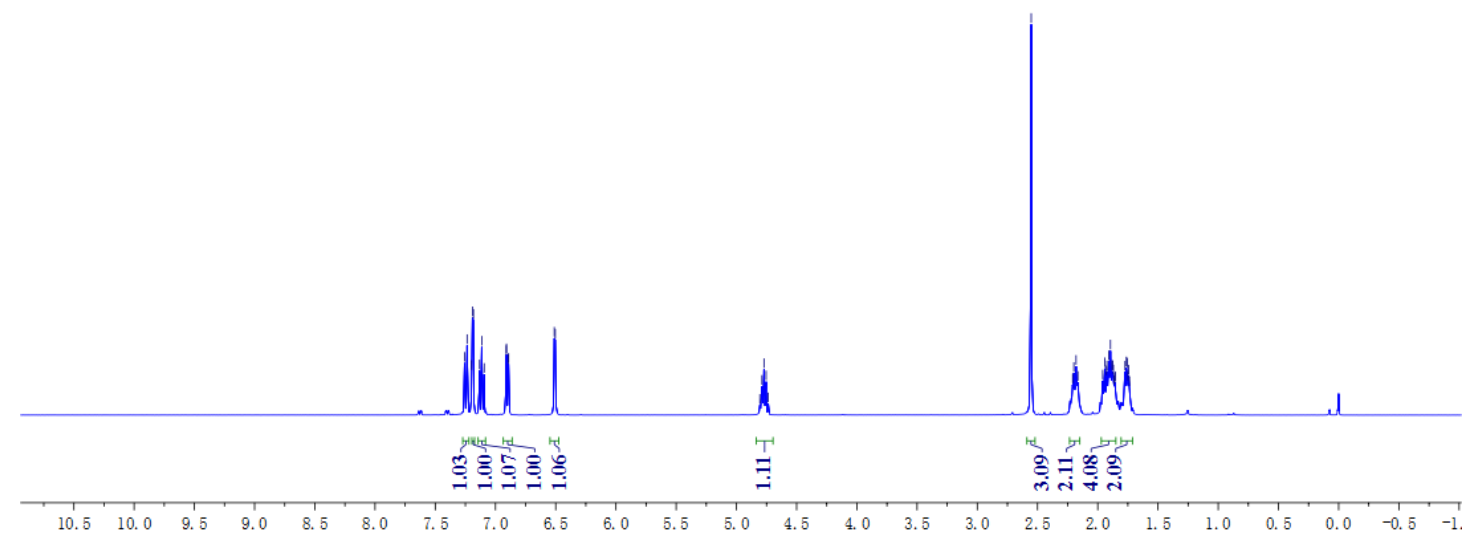

${ }^{13} \mathrm{C}$ NMR spectrum of compound $\mathbf{3 d}\left(\mathrm{CDCl}_{3}, 101 \mathrm{MHz}\right)$

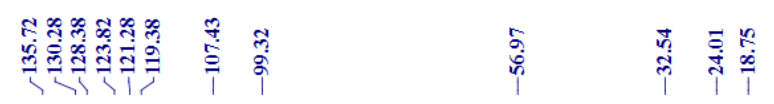

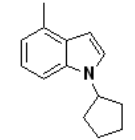

${ }^{13} \mathrm{C}$ NMR $(101 \mathrm{MHz}, \mathrm{CDCb})$

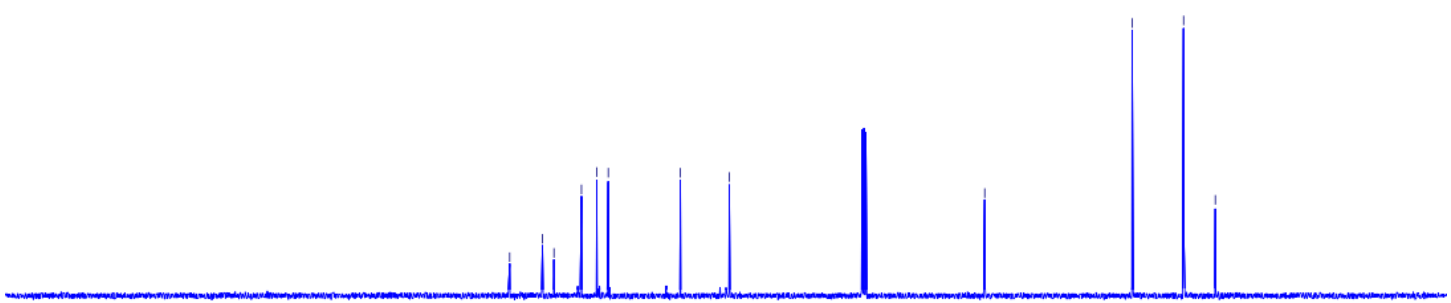

$\begin{array}{lllllllllllllllllllllll}1 & 1 \\ 210 & 200 & 190 & 180 & 170 & 160 & 150 & 140 & 130 & 120 & 110 & 100 & 90 & 80 & 70 & 60 & 50 & 40 & 30 & 20 & 10 & 0 & -10\end{array}$ 
${ }^{1} \mathrm{H}$ NMR spectrum of compound $\mathbf{3 e}\left(\mathrm{CDCl}_{3}, 400 \mathrm{MHz}\right)$

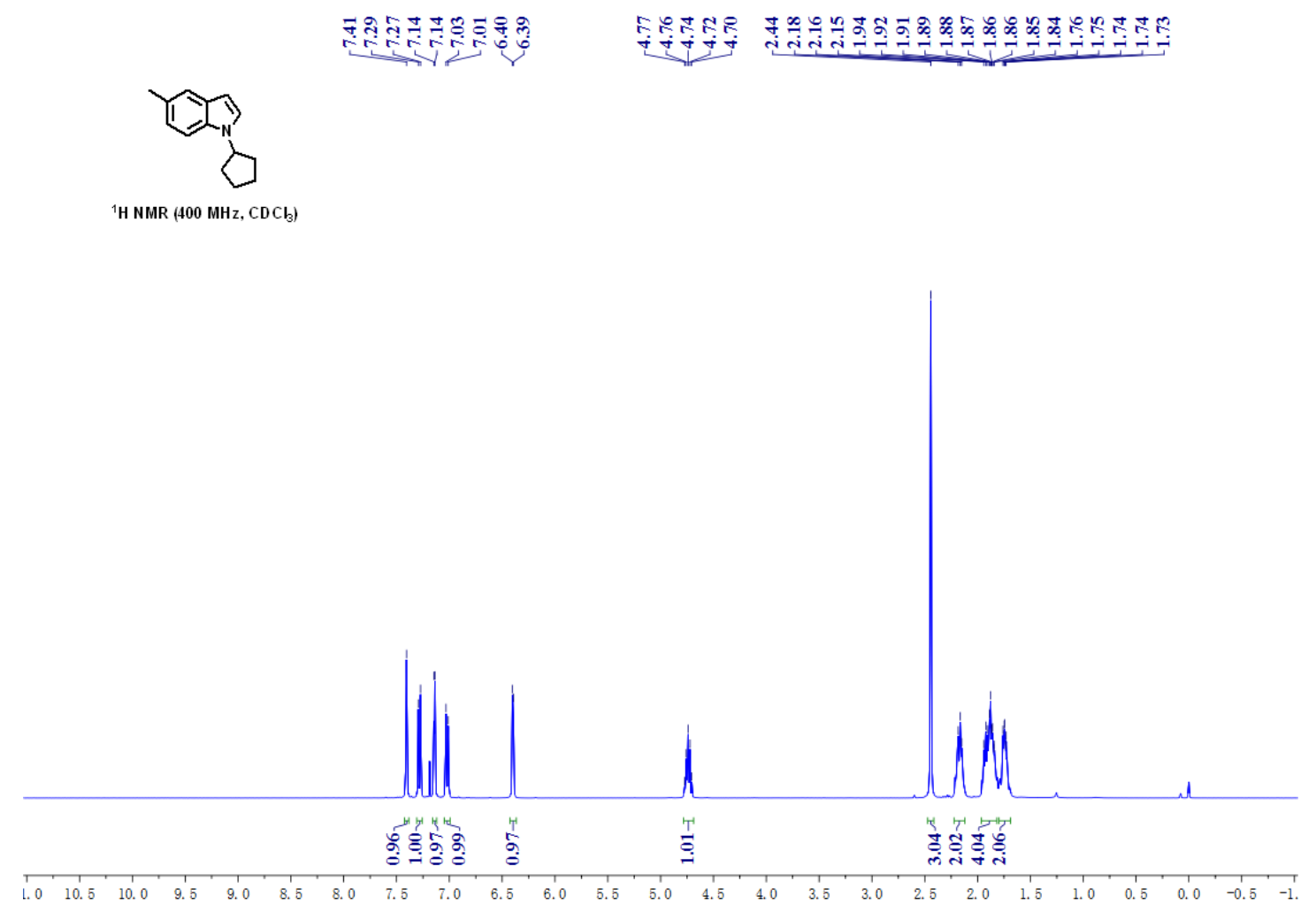

${ }^{13} \mathrm{C}$ NMR spectrum of compound $\mathbf{3 e}\left(\mathrm{CDCl}_{3}, 101 \mathrm{MHz}\right)$
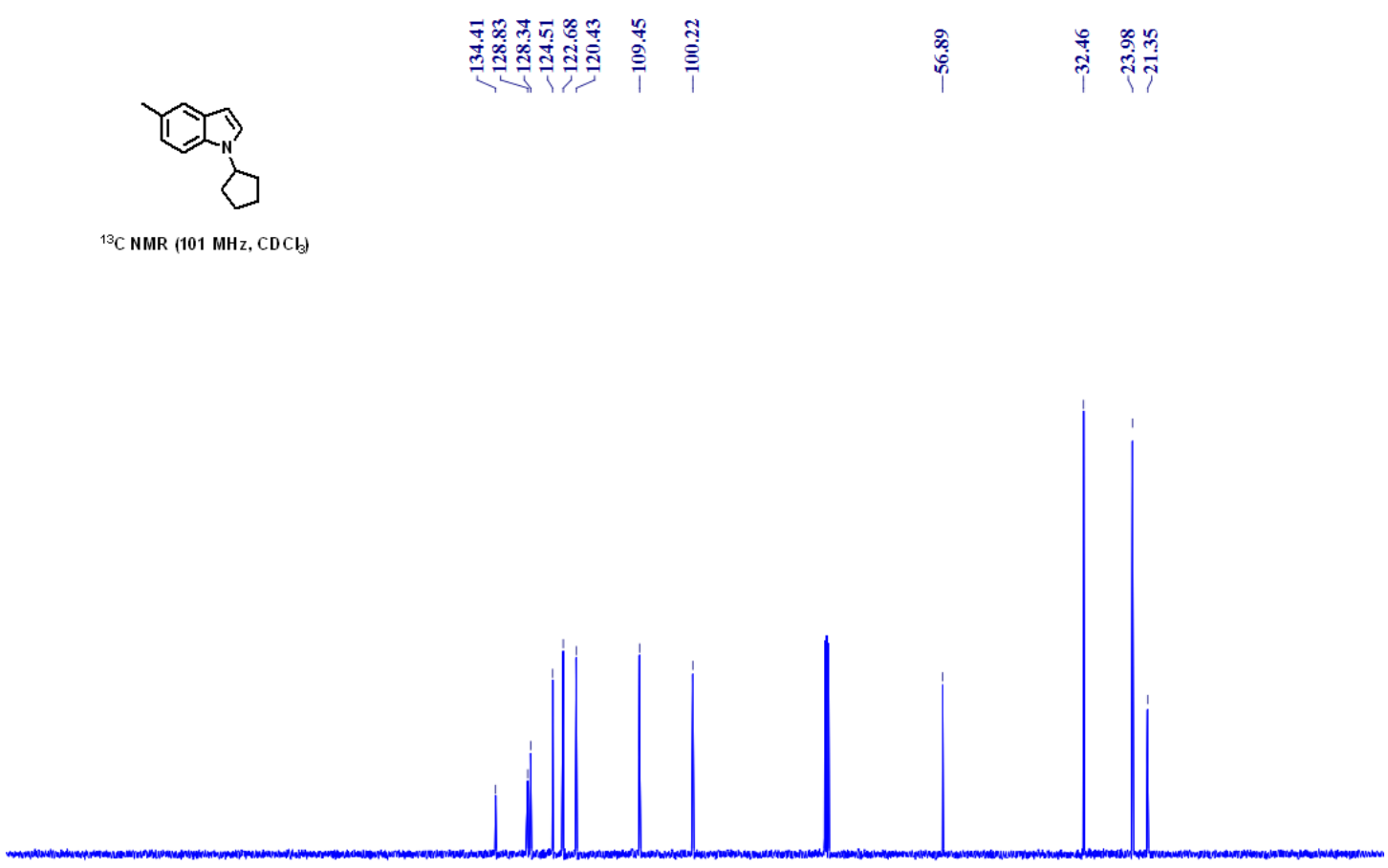

$\begin{array}{llllllllllllllllllllllllllll}1 & 1 & 200 & 190 & 180 & 170 & 160 & 150 & 140 & 130 & 120 & 110 & 100 & 90 & 80 & 70 & 60 & 50 & 10 & 10 & 20 & 10 & 0 & -10\end{array}$ 
${ }^{1} \mathrm{H}$ NMR spectrum of compound $\mathbf{3 f}\left(\mathrm{CDCl}_{3}, 400 \mathrm{MHz}\right)$

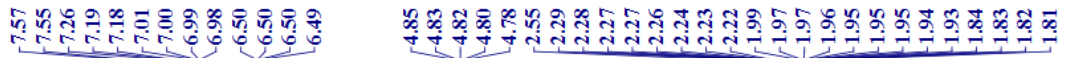

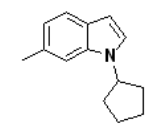

${ }^{1} \mathrm{H}$ NMR $\left(400 \mathrm{MHz}, \mathrm{CDCl}_{3}\right)$

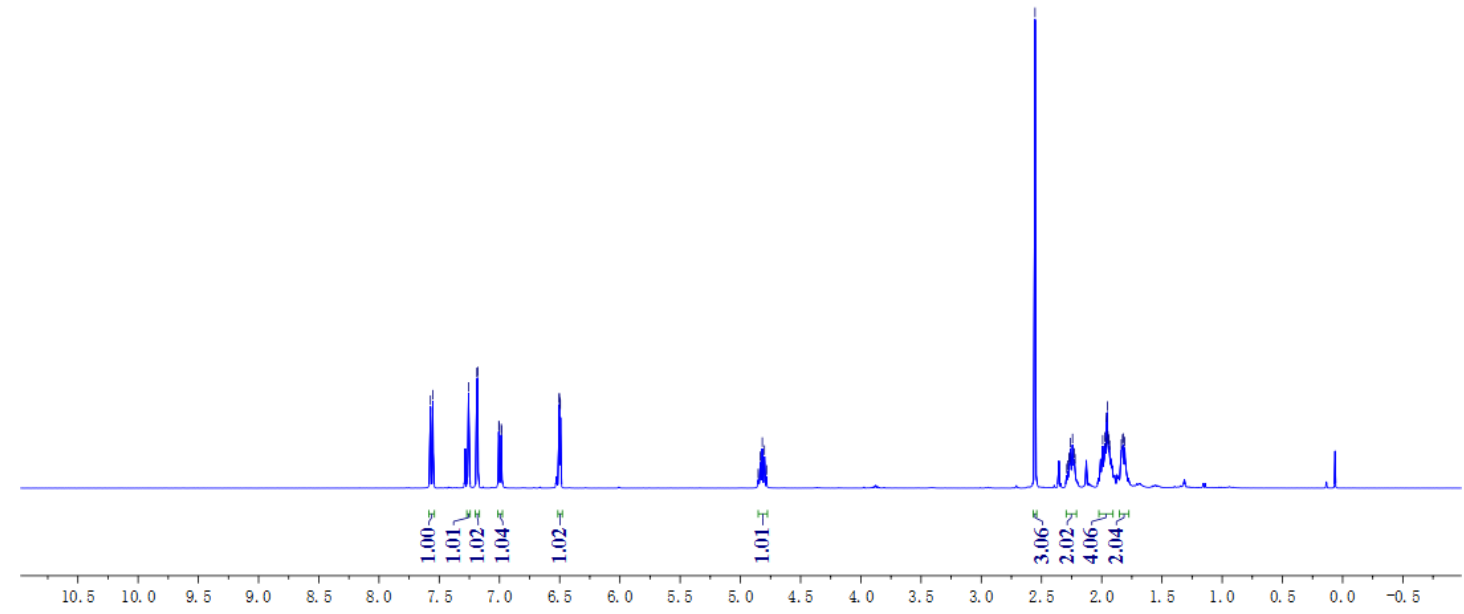

${ }^{13} \mathrm{C}$ NMR spectrum of compound $\mathbf{3 f}\left(\mathrm{CDCl}_{3}, 101 \mathrm{MHz}\right)$
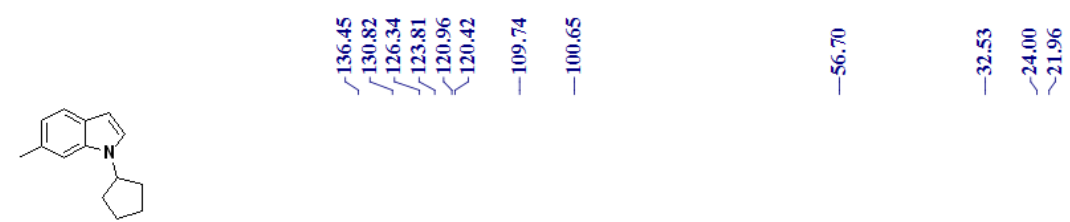

${ }^{13} \mathrm{C}$ NMR $(101 \mathrm{MHz}, \mathrm{CDCl})$

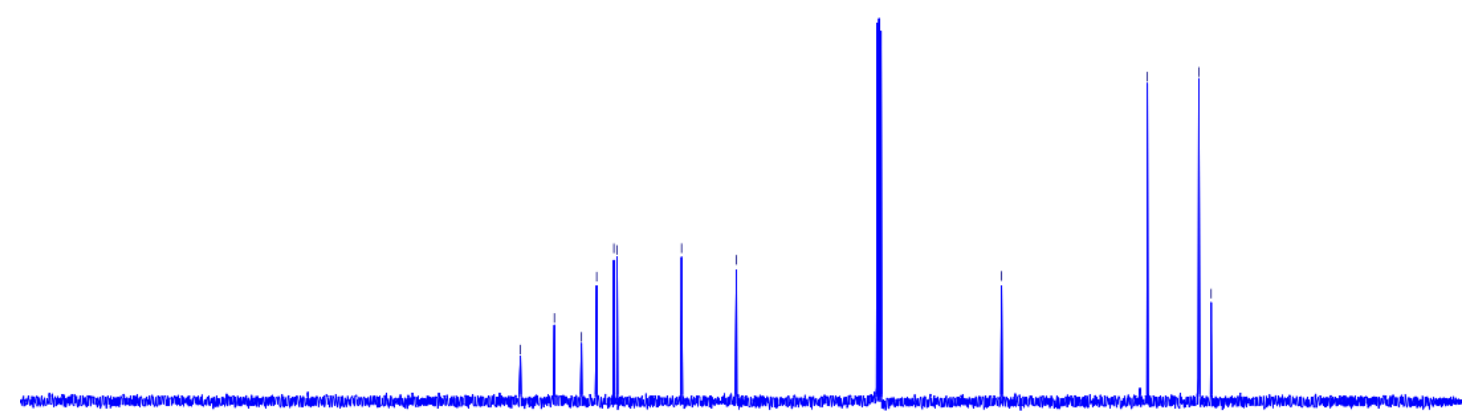

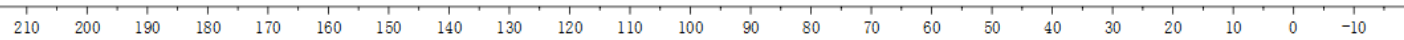


${ }^{1} \mathrm{H}$ NMR spectrum of compound $\mathbf{3 g}\left(\mathrm{CDCl}_{3}, 400 \mathrm{MHz}\right)$

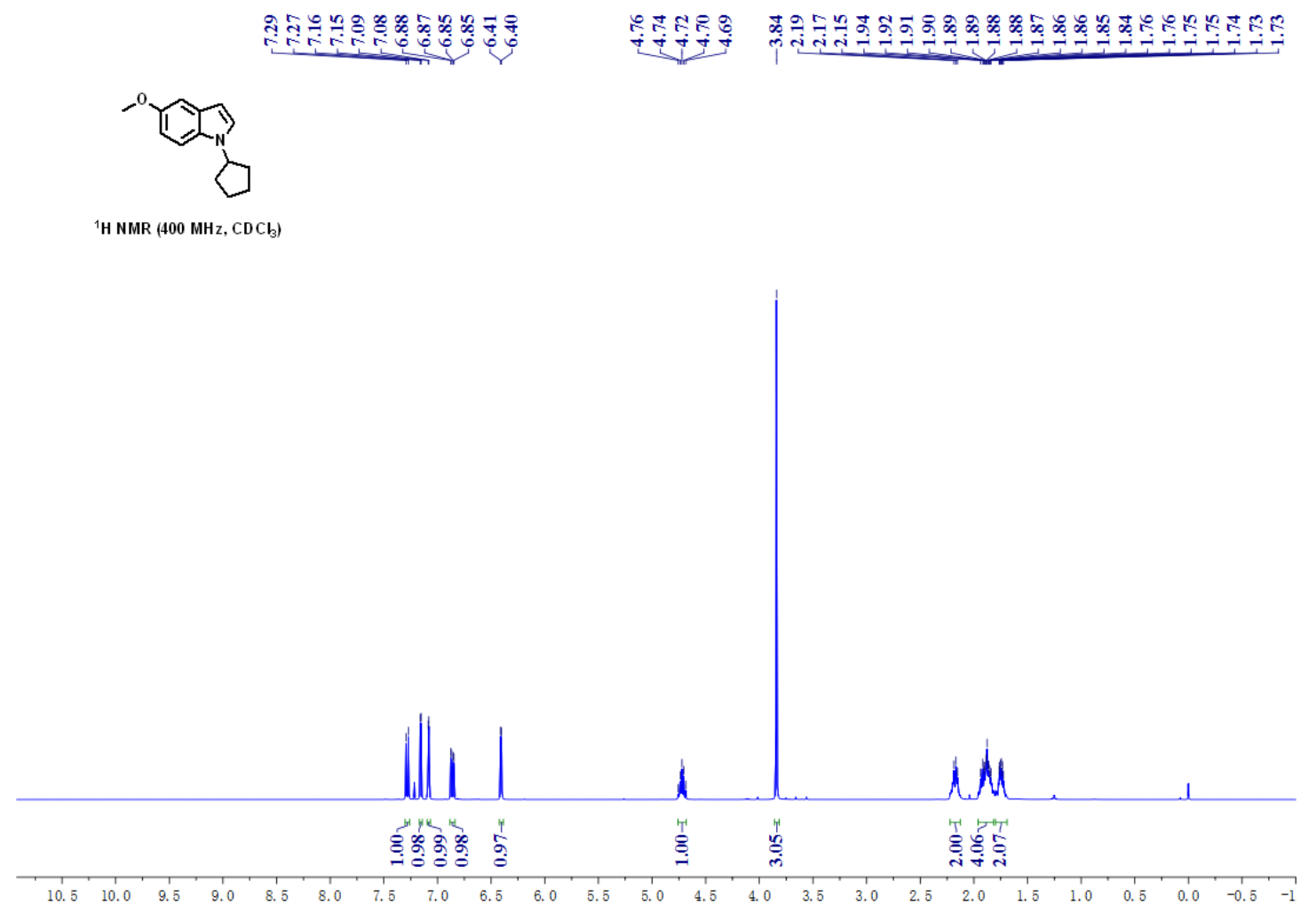

${ }^{13} \mathrm{C}$ NMR spectrum of compound $\mathbf{3 g}\left(\mathrm{CDCl}_{3}, 101 \mathrm{MHz}\right)$
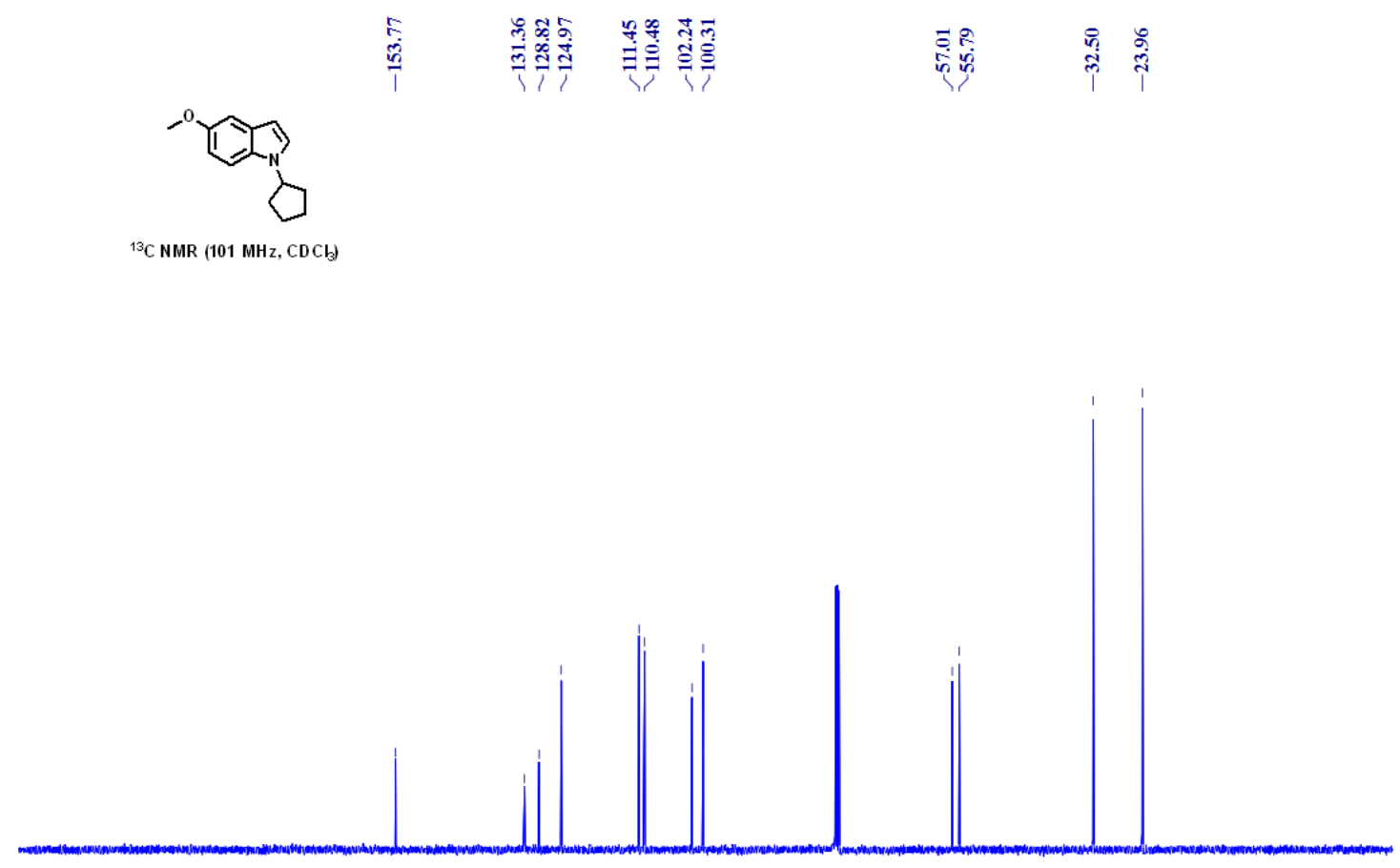

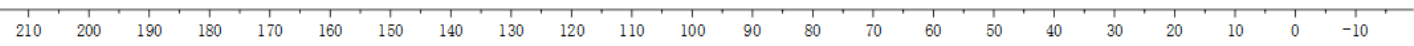


${ }^{1} \mathrm{H}$ NMR spectrum of compound $\mathbf{3 h}\left(\mathrm{CDCl}_{3}, 400 \mathrm{MHz}\right)$

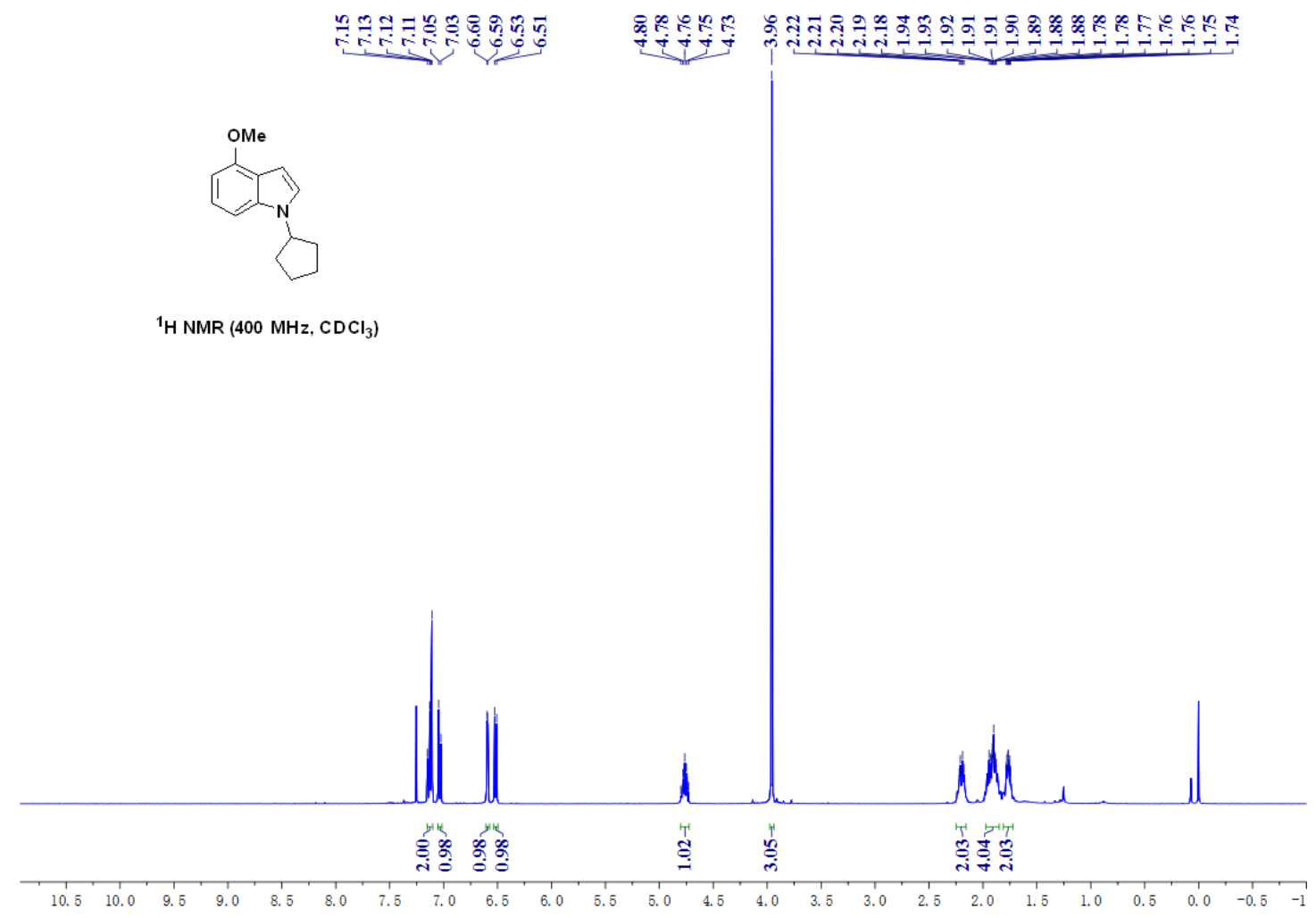

${ }^{13} \mathrm{C}$ NMR spectrum of compound $\mathbf{3 h}\left(\mathrm{CDCl}_{3}, 101 \mathrm{MHz}\right)$

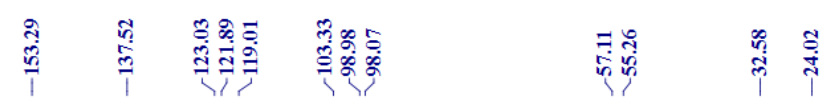

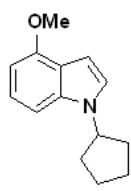

$\left.{ }^{13} \mathrm{C} \mathrm{NMR} \mathrm{(101} \mathrm{MHz,} \mathrm{CDCl}_{3}\right)$

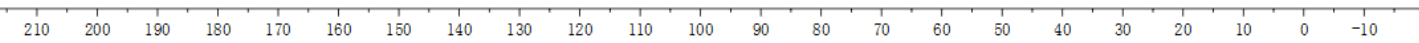


${ }^{1} \mathrm{H}$ NMR spectrum of compound $\mathbf{3 i}\left(\mathrm{CDCl}_{3}, 400 \mathrm{MHz}\right)$
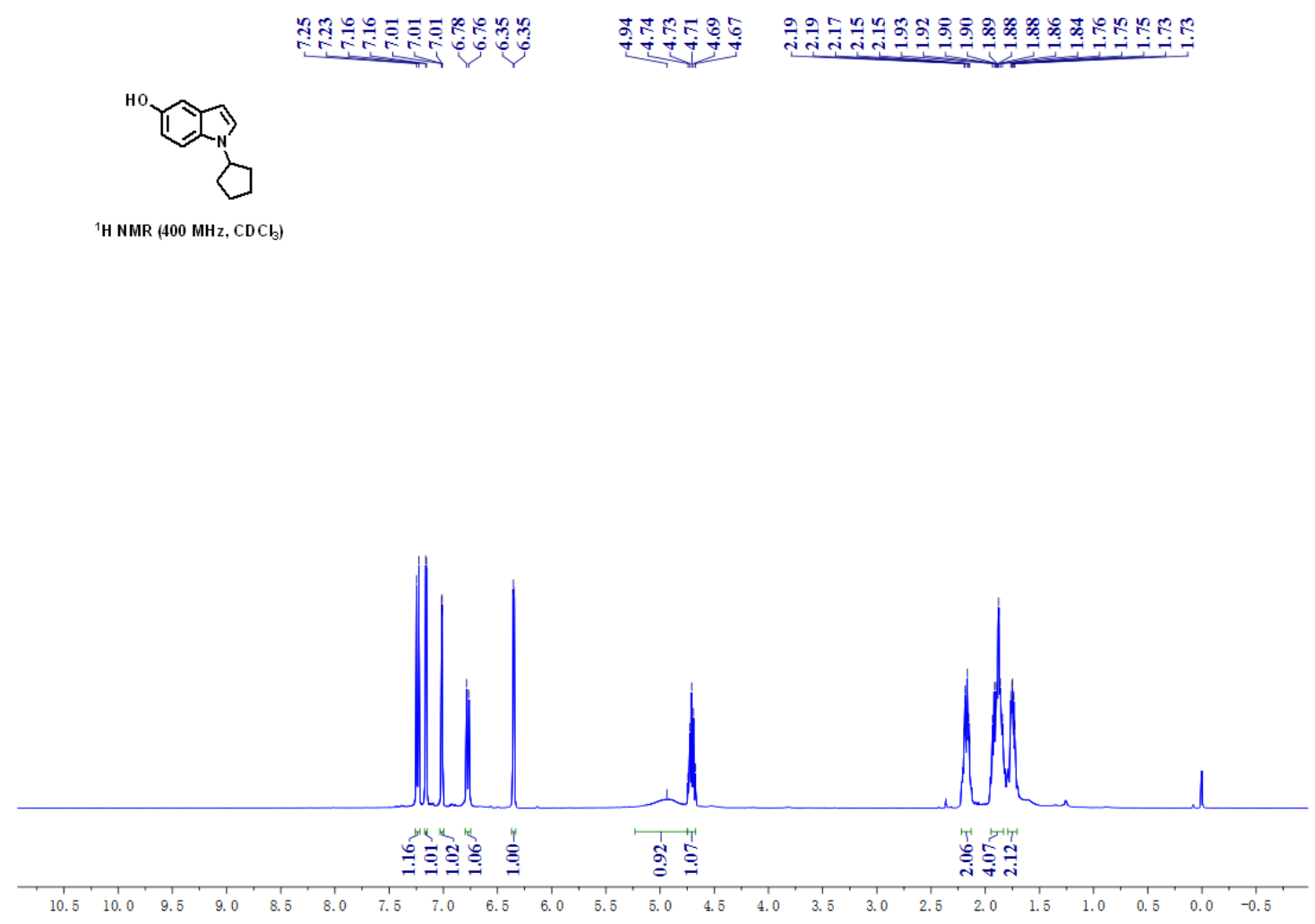

${ }^{13} \mathrm{C}$ NMR spectrum of compound $\mathbf{3 i}\left(\mathrm{CDCl}_{3}, 101 \mathrm{MHz}\right)$
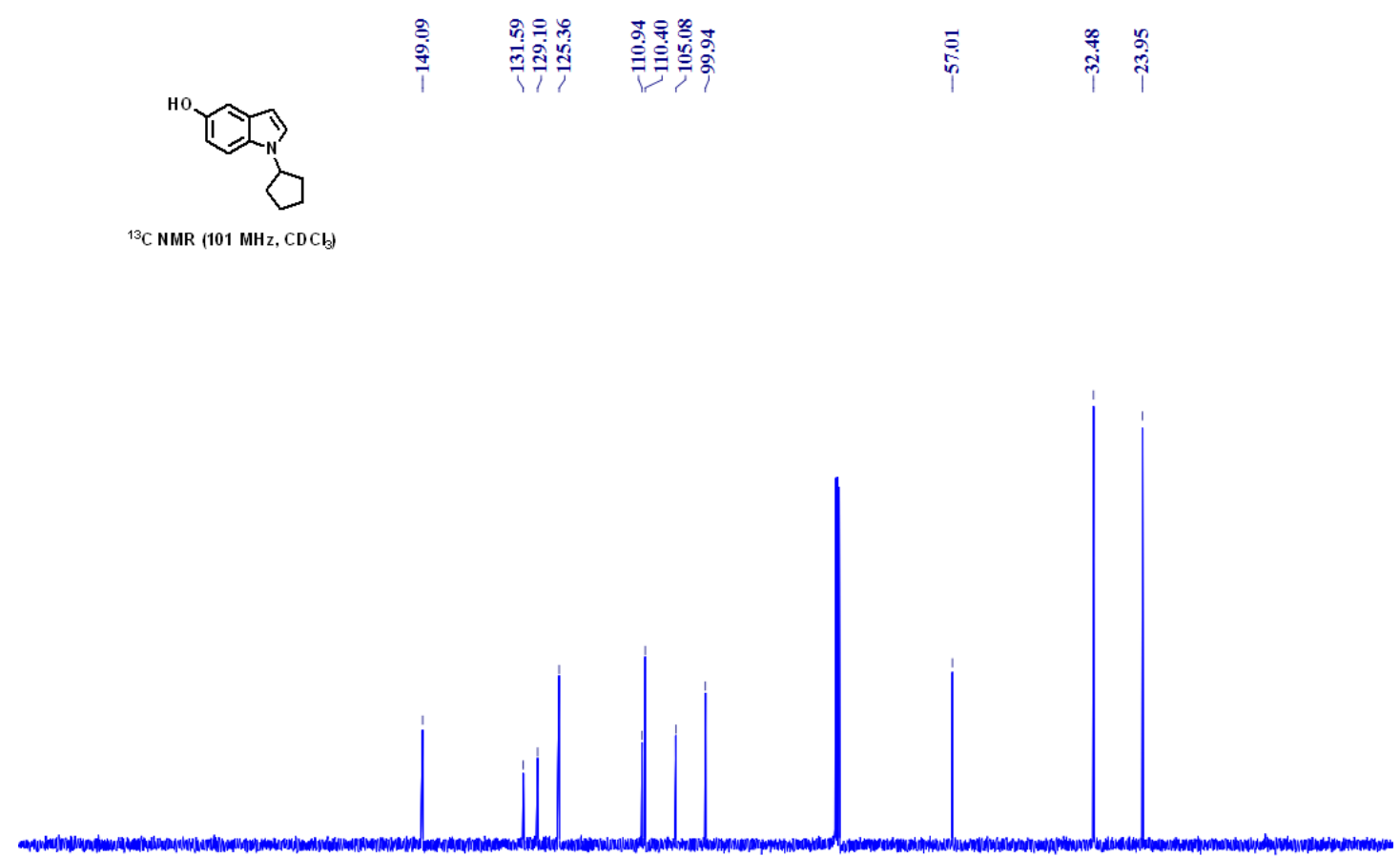

${ }_{210}^{1} \quad \begin{array}{lllllllllllllllllllllllllll}1 & 1 & 1 & 1 & 1 & 1 & 1 & 170 & 160 & 150 & 140 & 130 & 120 & 110 & 100 & 90 & 80 & 70 & 60 & 50 & 10 & 30 & 20 & 10 & 0 & -10\end{array}$ 
${ }^{1} \mathrm{H}$ NMR spectrum of compound $\mathbf{3 j}\left(\mathrm{CDCl}_{3}, 400 \mathrm{MHz}\right)$

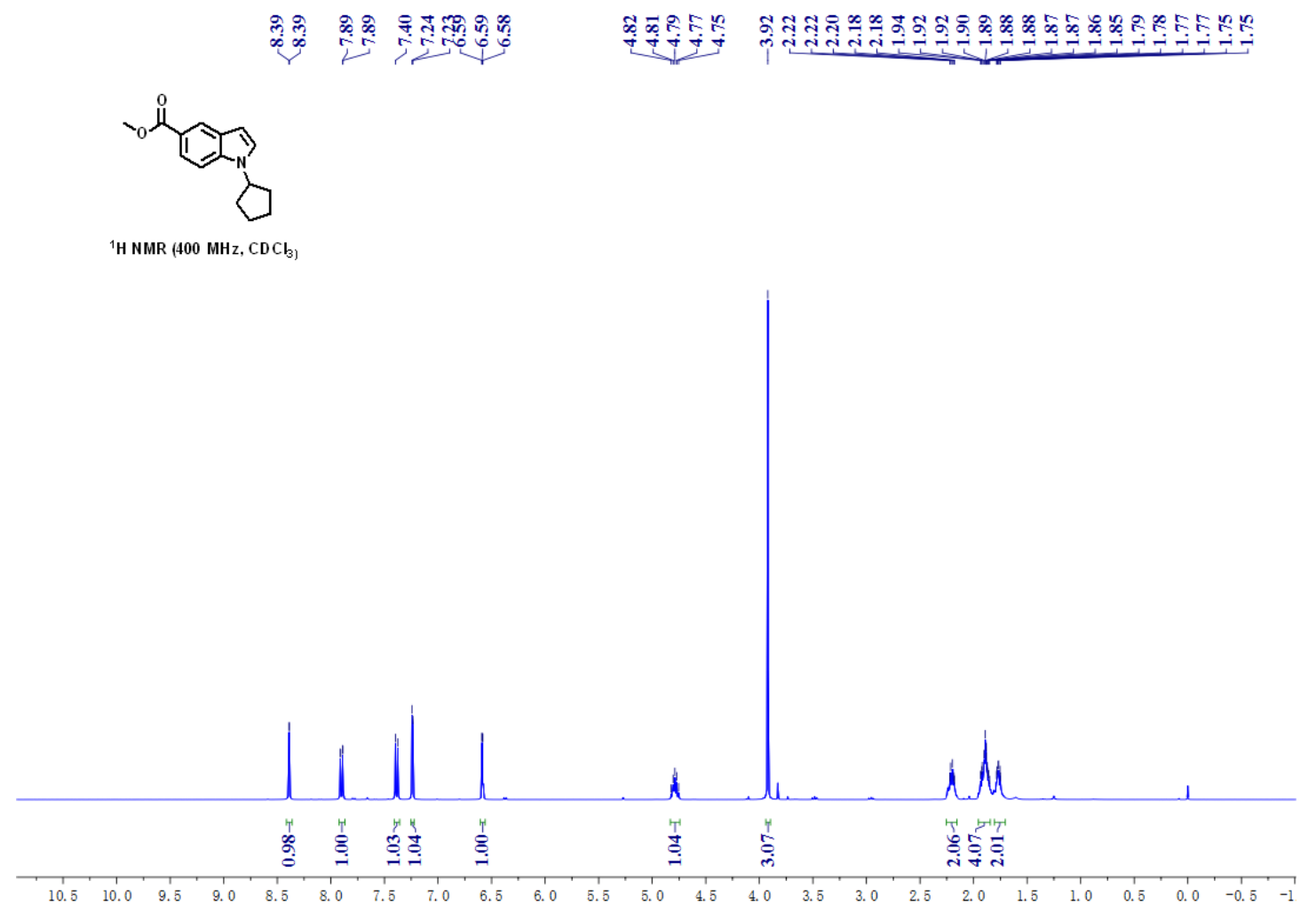

${ }^{13} \mathrm{C}$ NMR spectrum of compound $\mathbf{3 j}\left(\mathrm{CDCl}_{3}, 101 \mathrm{MHz}\right)$
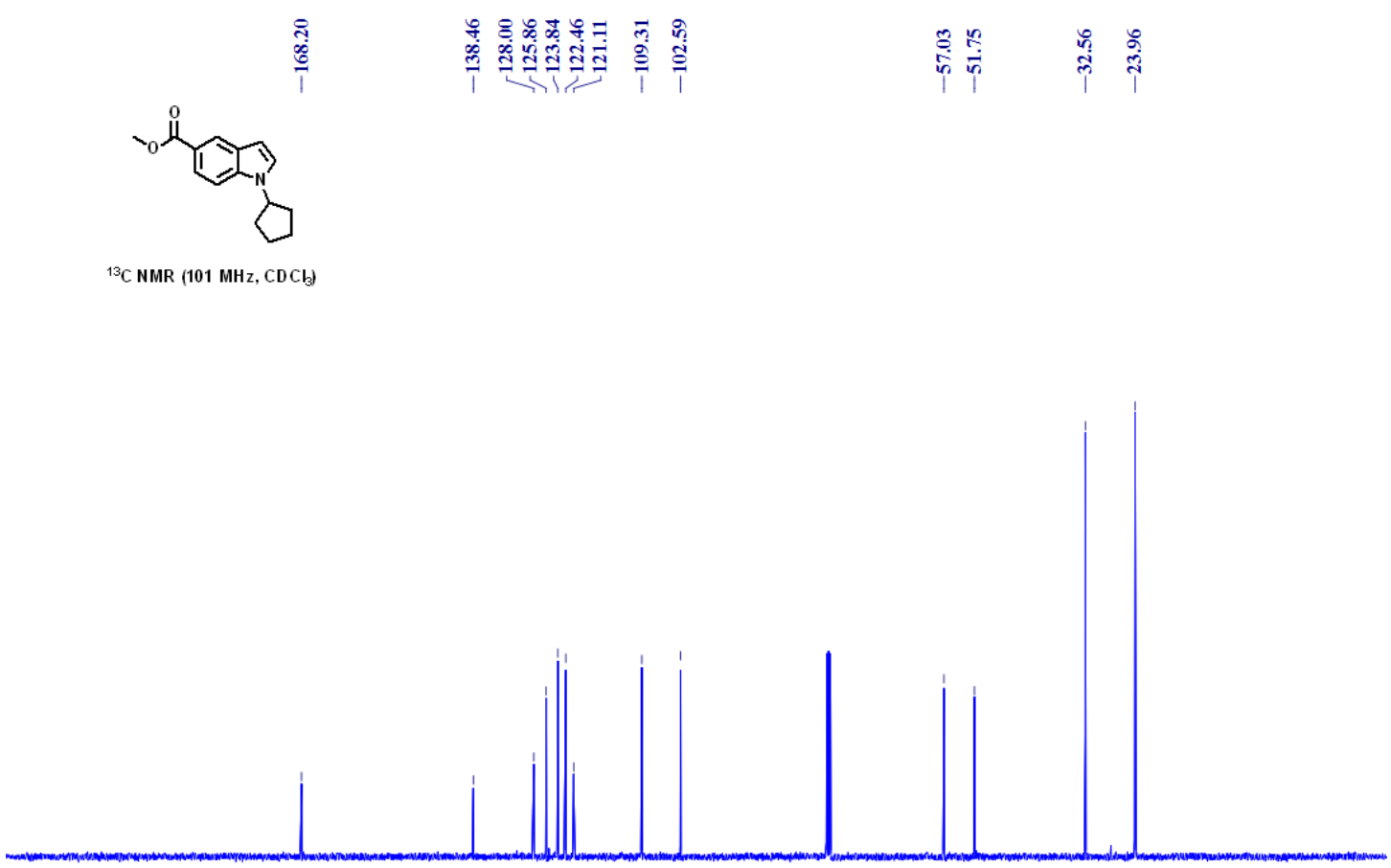

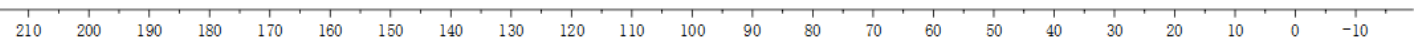


${ }^{1} \mathrm{H}$ NMR spectrum of compound $\mathbf{3 k}\left(\mathrm{CDCl}_{3}, 400 \mathrm{MHz}\right)$
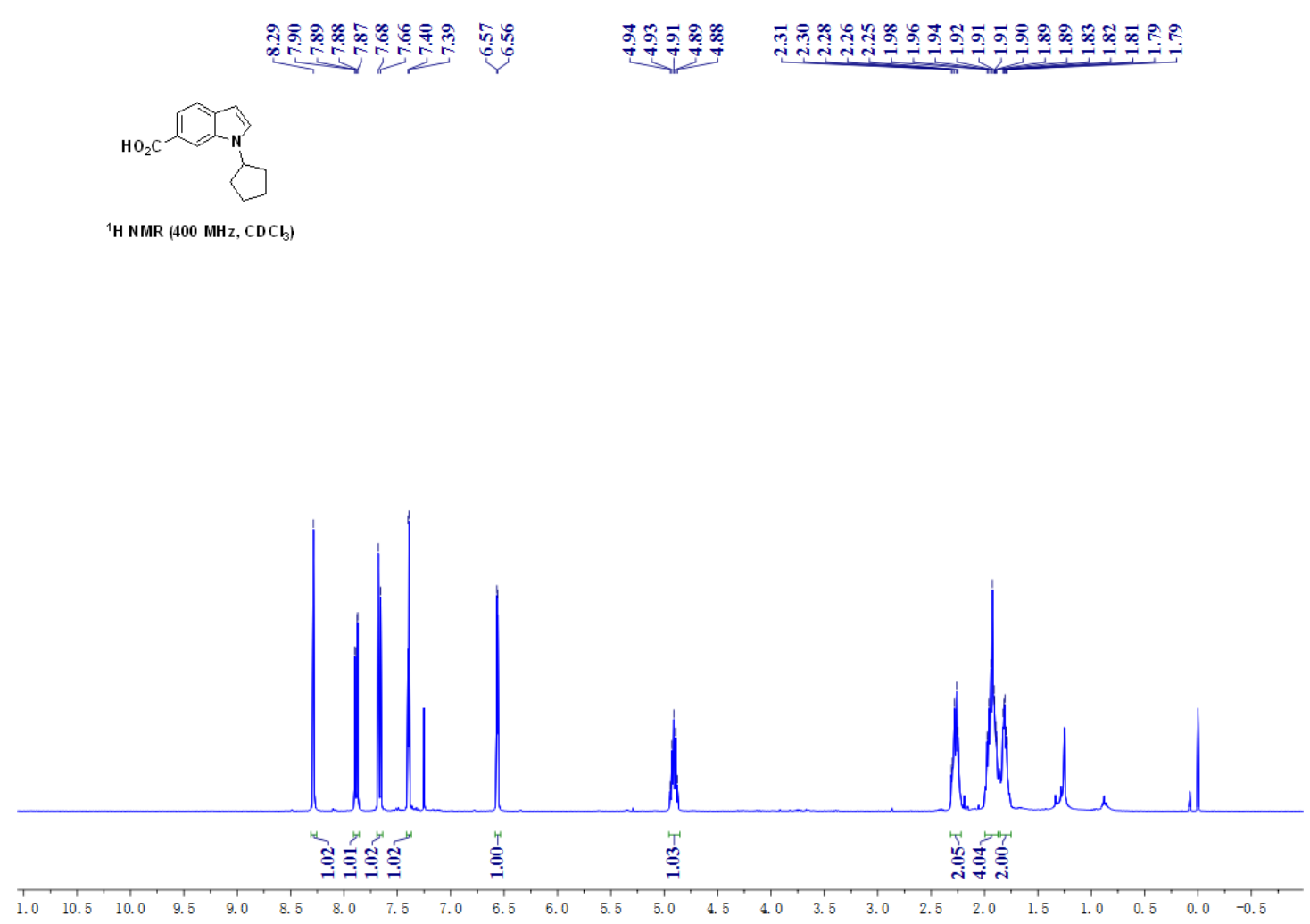

${ }^{13} \mathrm{C}$ NMR spectrum of compound $\mathbf{3 k}\left(\mathrm{CDCl}_{3}, 101 \mathrm{MHz}\right)$
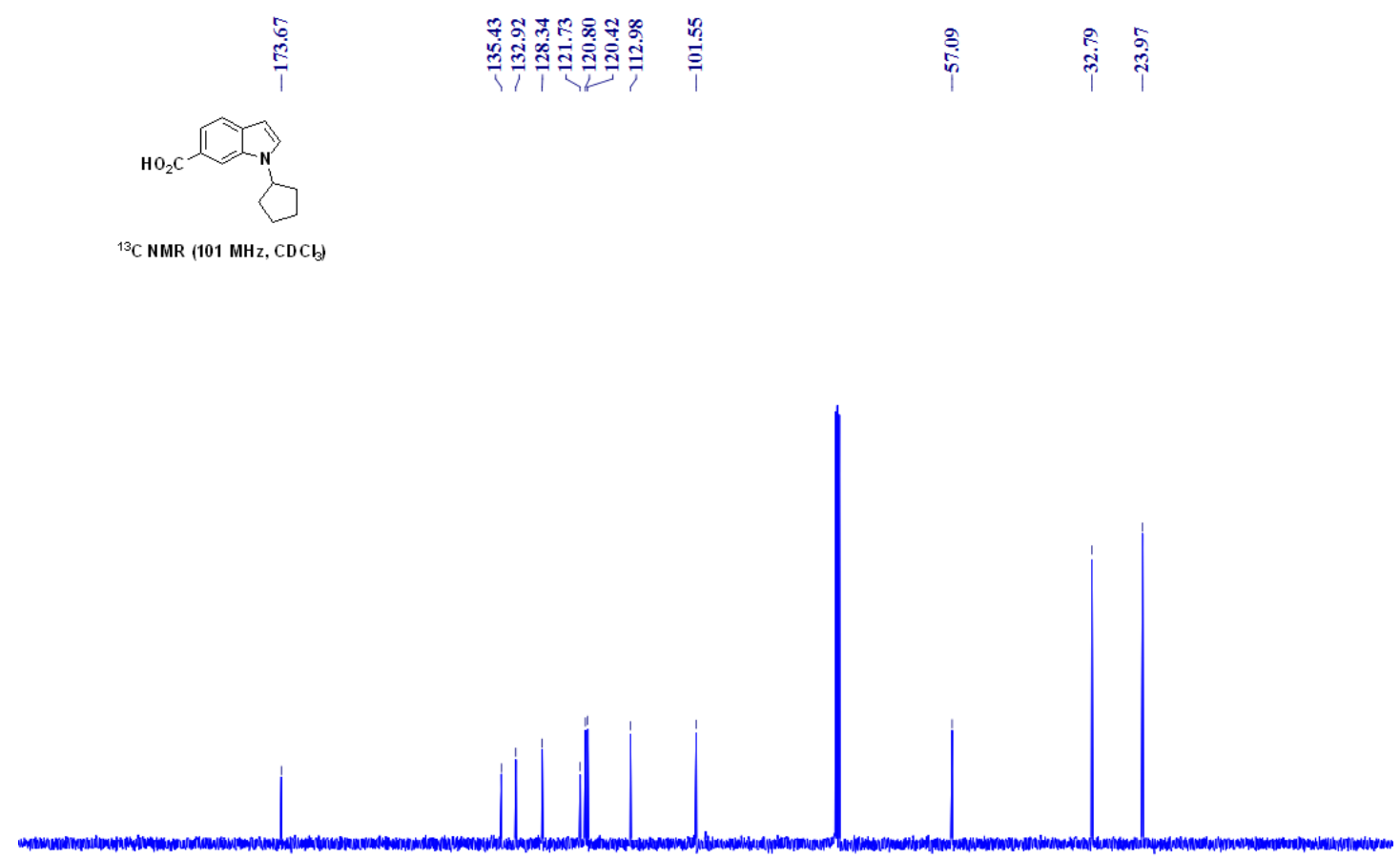

${ }_{210}^{1} \quad \begin{array}{llllllllllllllllllllllllllll}1 & 200 & 190 & 180 & 170 & 160 & 150 & 140 & 130 & 120 & 110 & 100 & 90 & 80 & 70 & 60 & 50 & 10 & 30 & 20 & 10 & 0 & -10\end{array}$ 
${ }^{1} \mathrm{H}$ NMR spectrum of compound $31\left(\mathrm{CDCl}_{3}, 600 \mathrm{MHz}\right)$
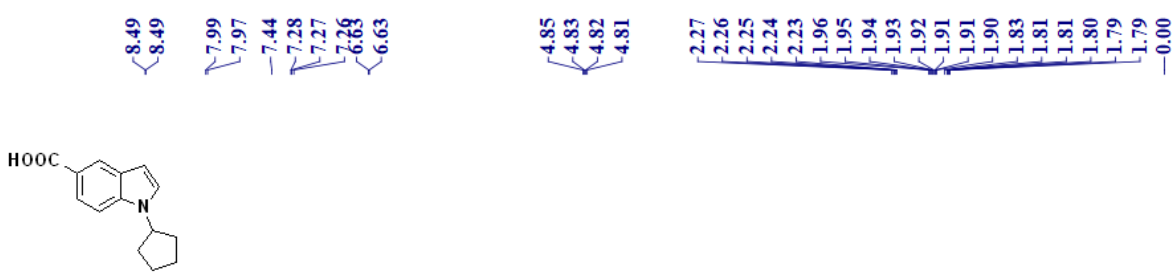

${ }^{1} \mathrm{H}$ NMR $(600 \mathrm{MHz}, \mathrm{CDCl})$

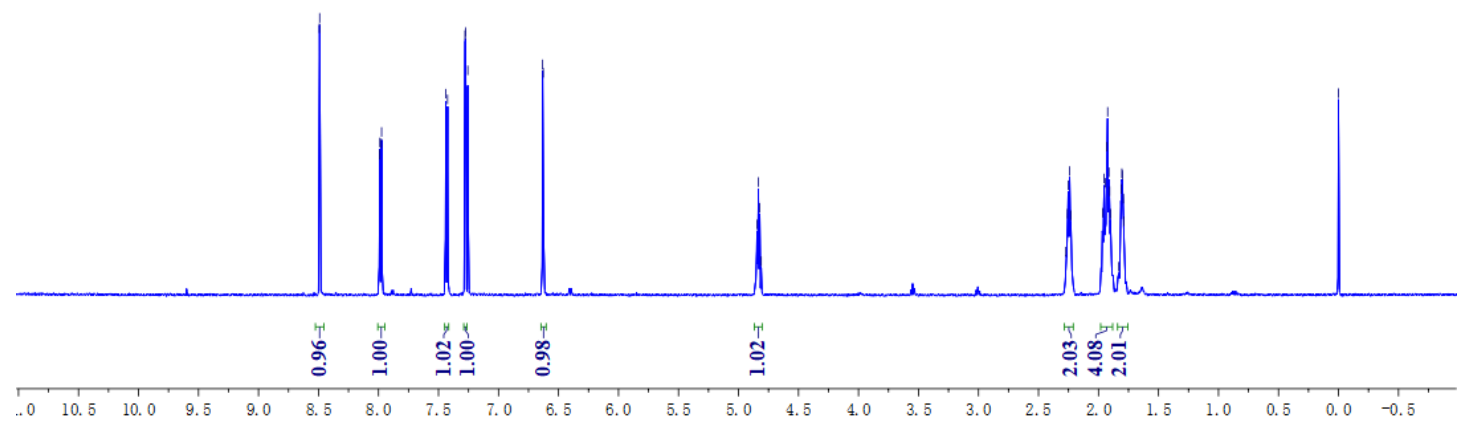

${ }^{13} \mathrm{C}$ NMR spectrum of compound $31\left(\mathrm{CDCl}_{3}, 151 \mathrm{MHz}\right)$
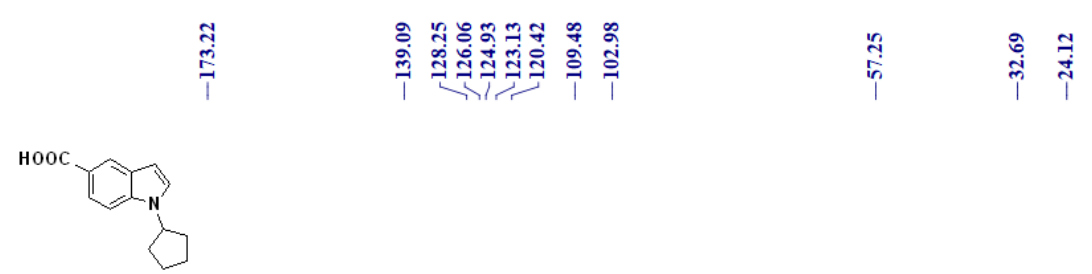

${ }^{13} \mathrm{C}$ NMR $(151 \mathrm{MHz}, \mathrm{CDCl})$

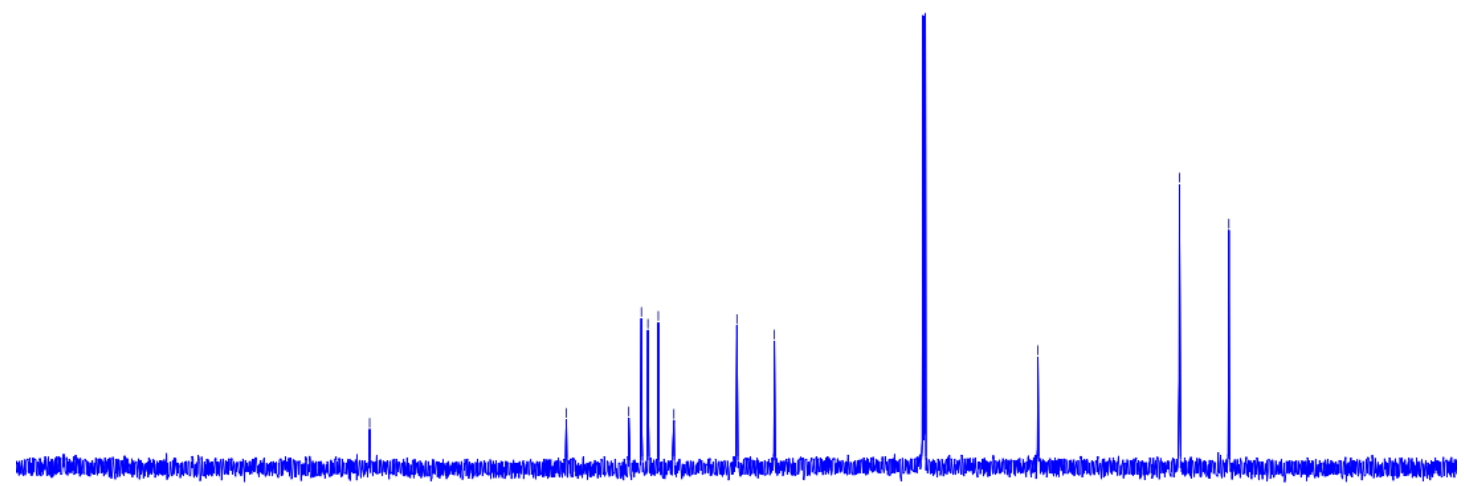

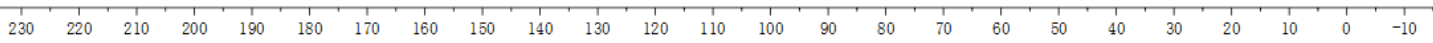


${ }^{1} \mathrm{H}$ NMR spectrum of compound $\mathbf{3} \mathbf{m}\left(\mathrm{CDCl}_{3}, 400 \mathrm{MHz}\right)$

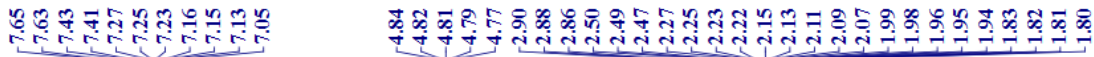

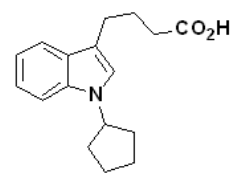

${ }^{1} \mathrm{H}$ NMR $\left(400 \mathrm{MHz}, \mathrm{CDCl}_{3}\right)$

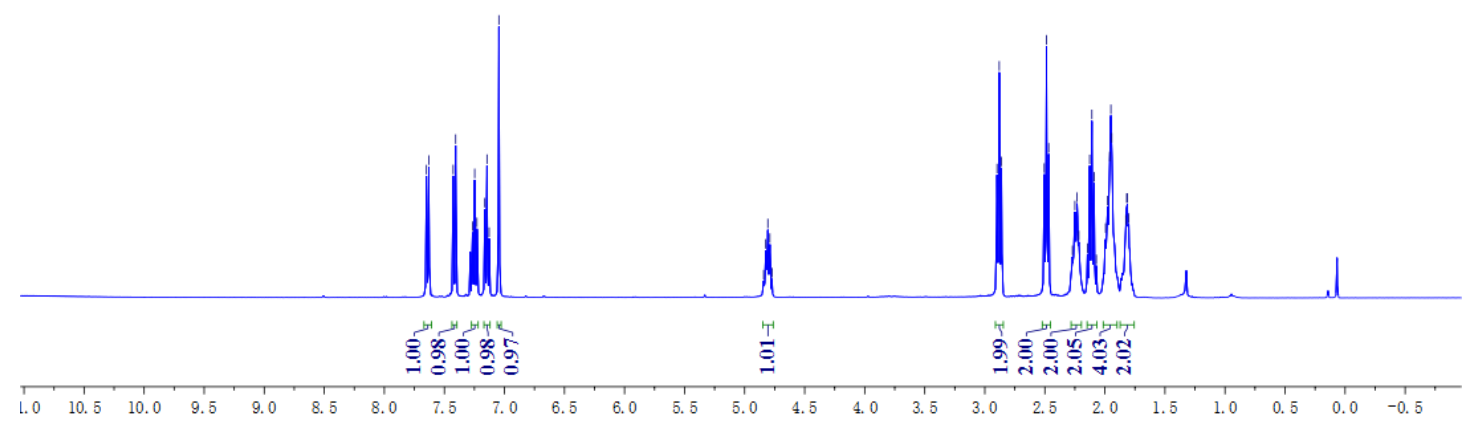

${ }^{13} \mathrm{C}$ NMR spectrum of compound $\mathbf{3 m}\left(\mathrm{CDCl}_{3}, 101 \mathrm{MHz}\right)$
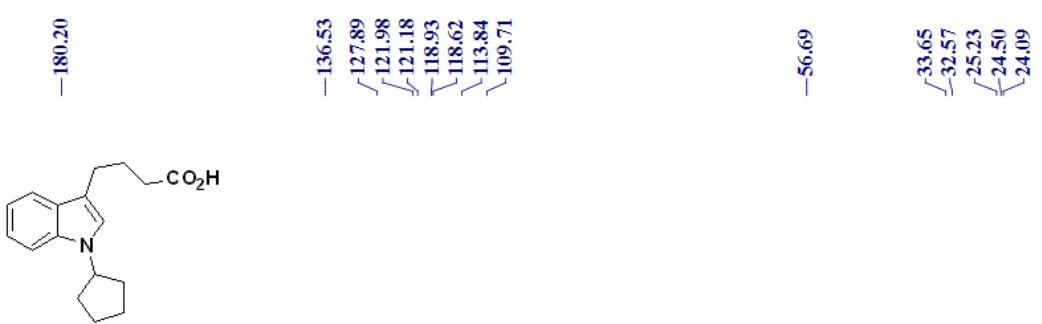

${ }^{13} \mathrm{C}$ NMR $\left(101 \mathrm{MHz}, \mathrm{CDCl}_{3}\right)$

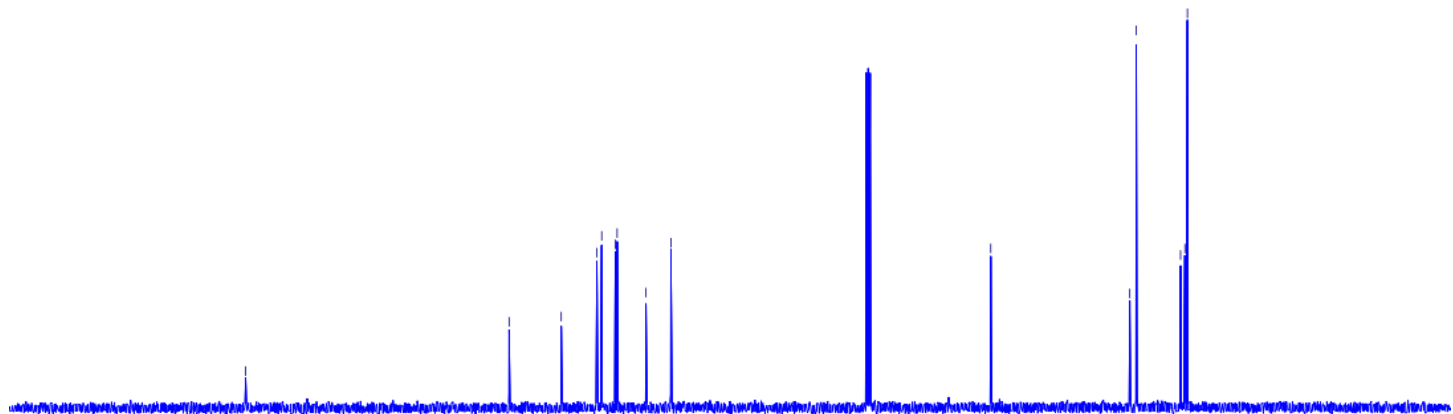

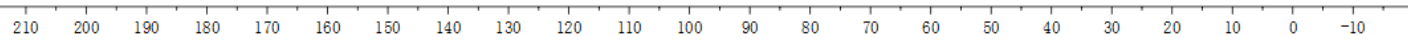


${ }^{1} \mathrm{H}$ NMR spectrum of compound $\mathbf{3 n}\left(\mathrm{CDCl}_{3}, 600 \mathrm{MHz}\right)$
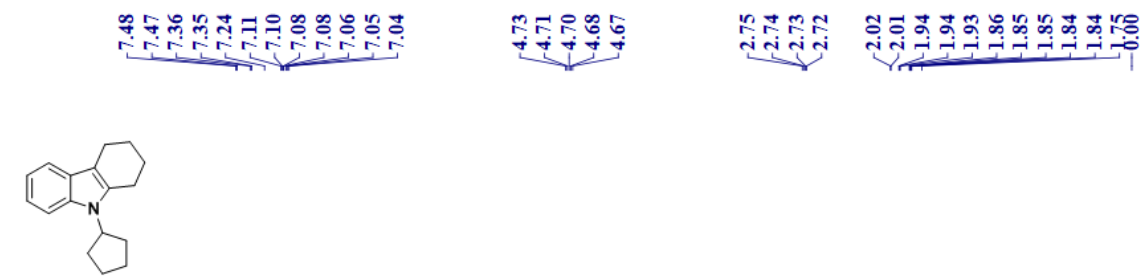

${ }^{1} \mathrm{H} \mathrm{NMR}\left(600 \mathrm{MHz}, \mathrm{CDCl}_{3}\right)$

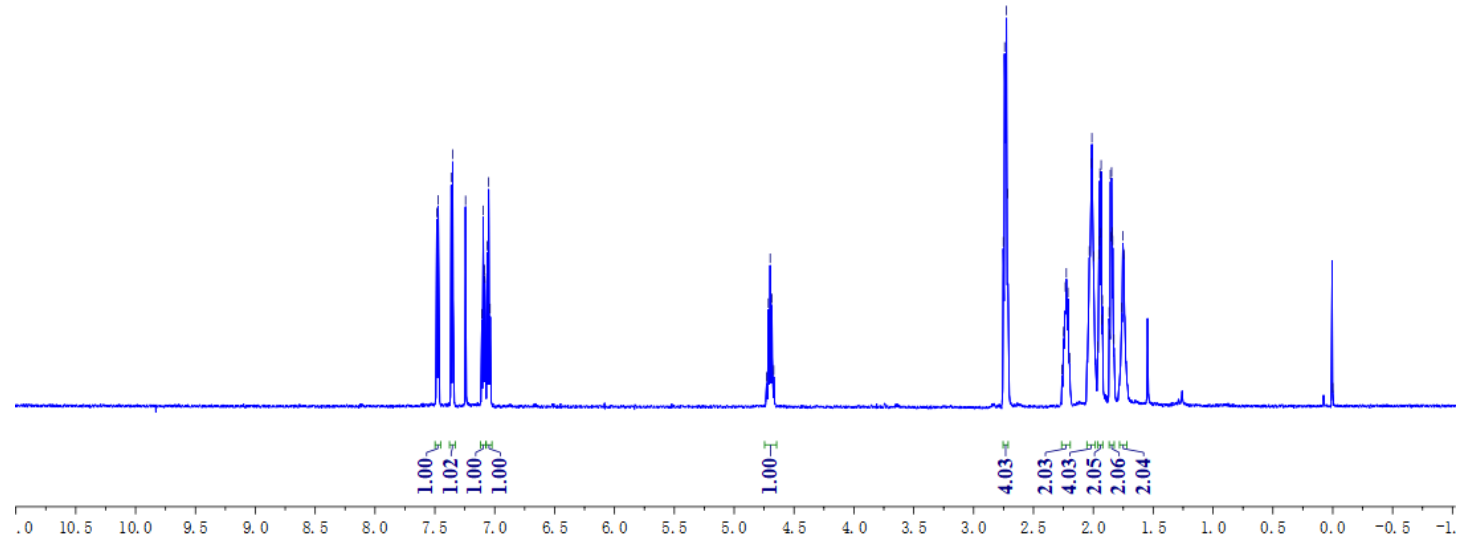

${ }^{13} \mathrm{C} \mathrm{NMR}$ spectrum of compound $3 \mathbf{n}\left(\mathrm{CDCl}_{3}, 151 \mathrm{MHz}\right)$
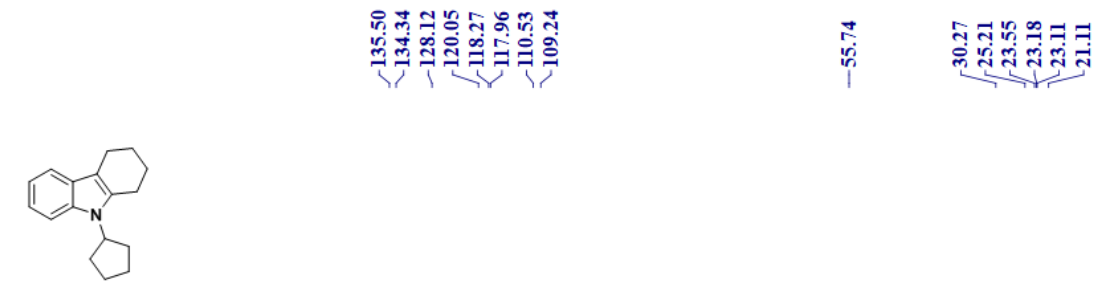

${ }^{13} \mathrm{C}$ NMR (151 MHz, $\left.\mathrm{CDCl}_{3}\right)$

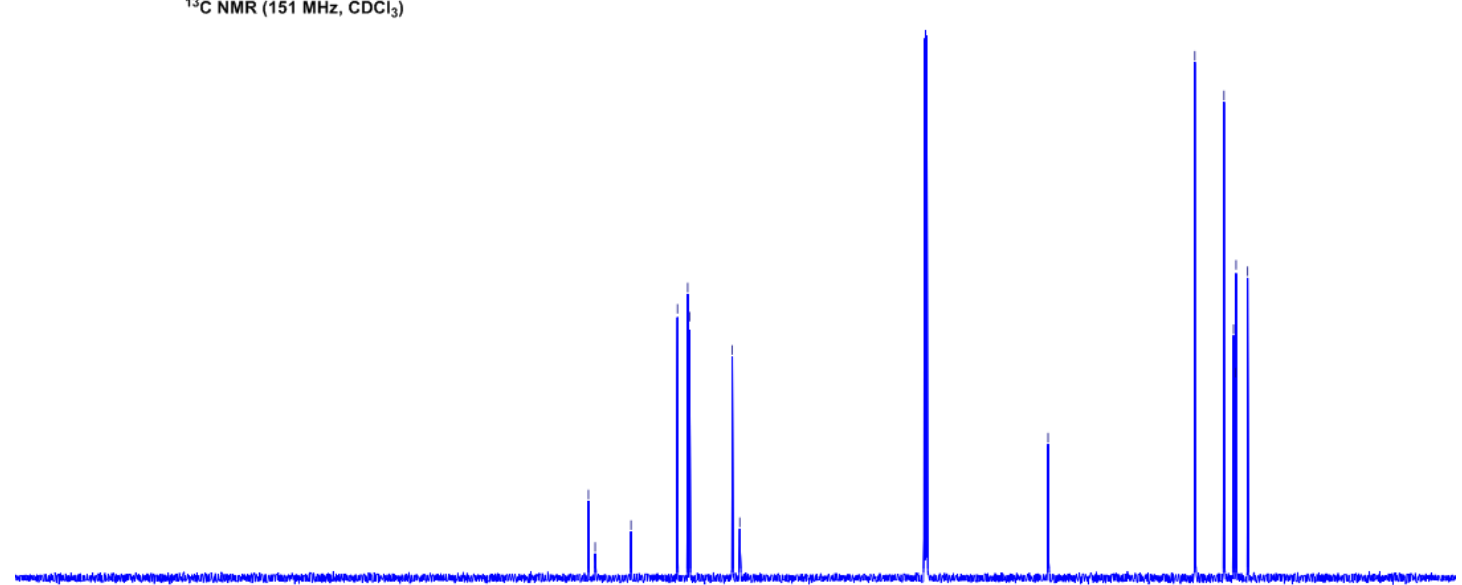

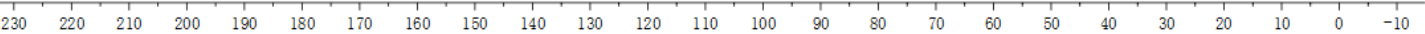


${ }^{1} \mathrm{H}$ NMR spectrum of compound $30\left(\mathrm{CDCl}_{3}, 400 \mathrm{MHz}\right)$

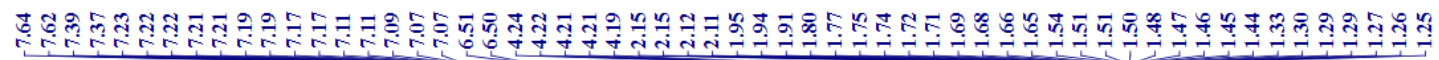

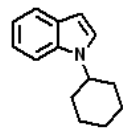

${ }^{1} \mathrm{H}$ NMR $(400 \mathrm{MHz}, \mathrm{CDCl})$

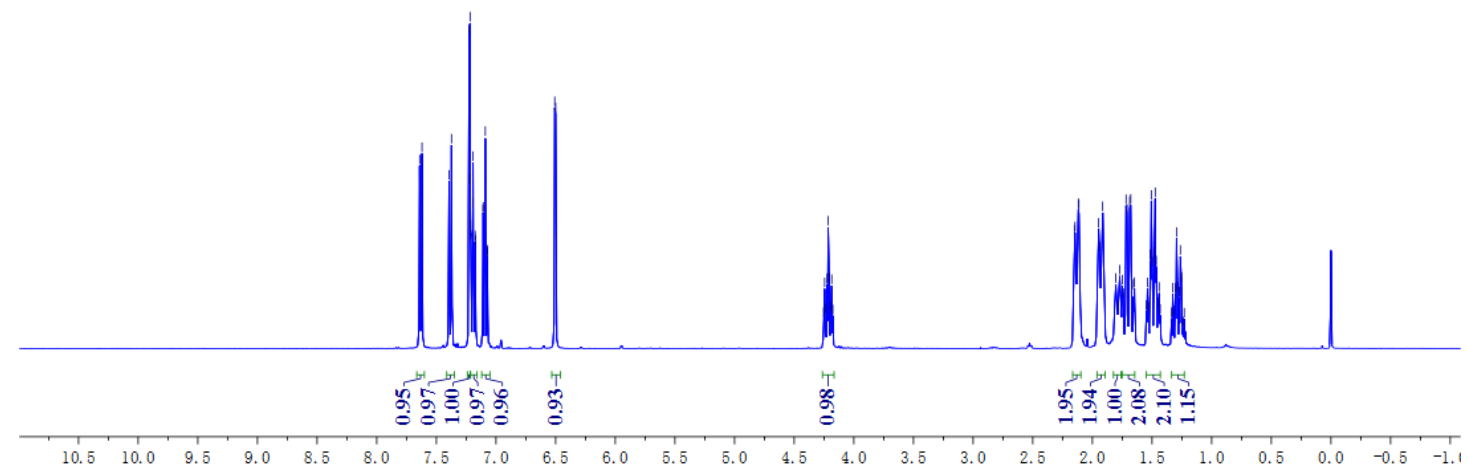

${ }^{13} \mathrm{C}$ NMR spectrum of compound $30\left(\mathrm{CDCl}_{3}, 101 \mathrm{MHz}\right)$
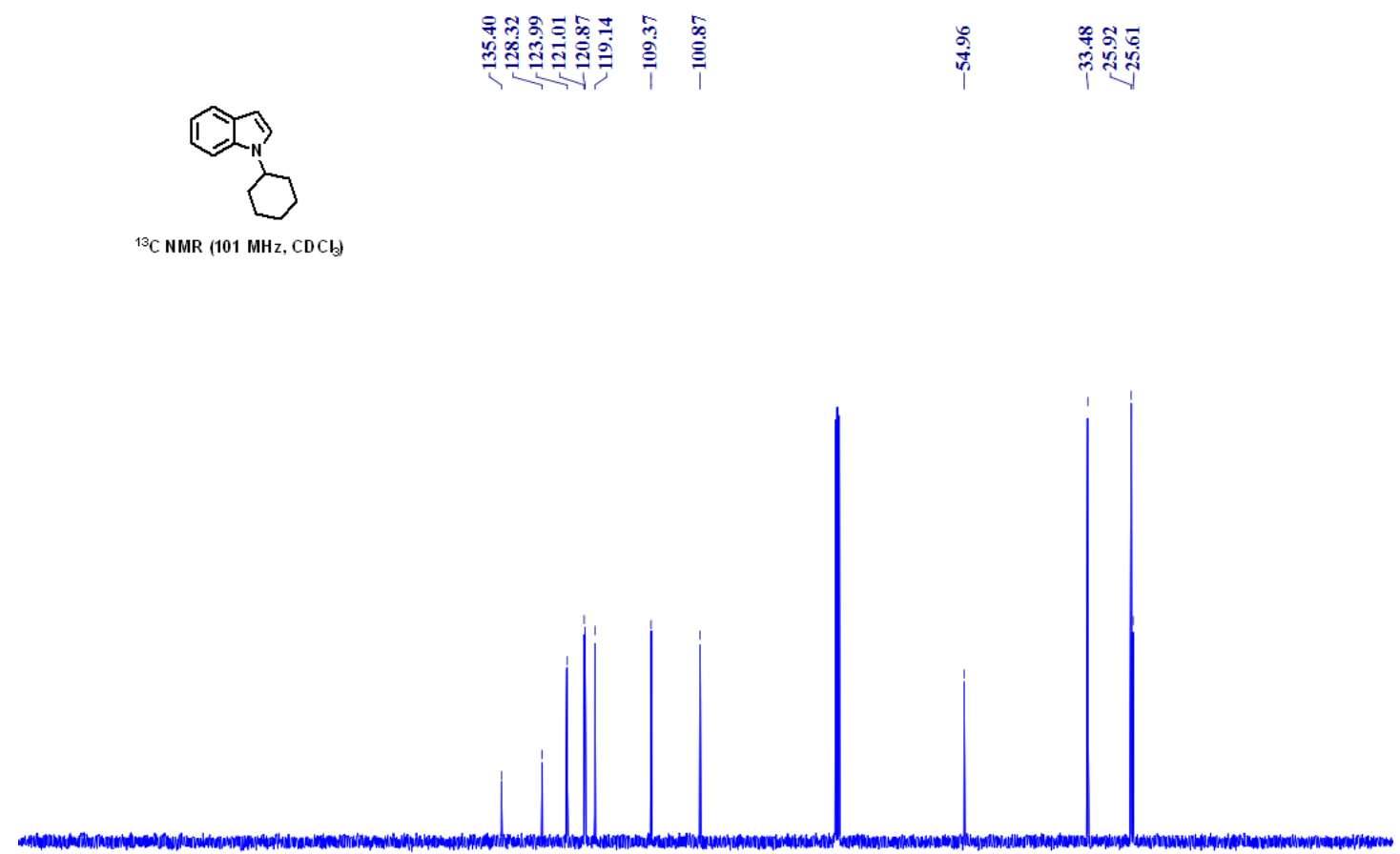

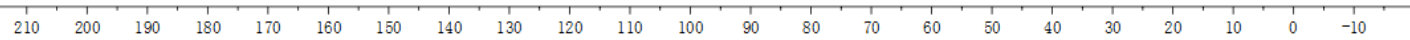


${ }^{1} \mathrm{H}$ NMR spectrum of compound $\mathbf{3 p}\left(\mathrm{CDCl}_{3}, 400 \mathrm{MHz}\right)$

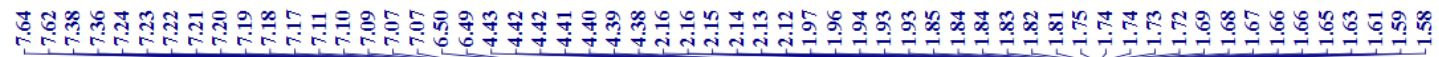

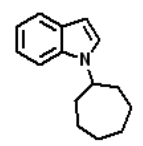

${ }^{1} \mathrm{H}$ NMR $\left(400 \mathrm{MHz}, \mathrm{CDCl}_{3}\right)$

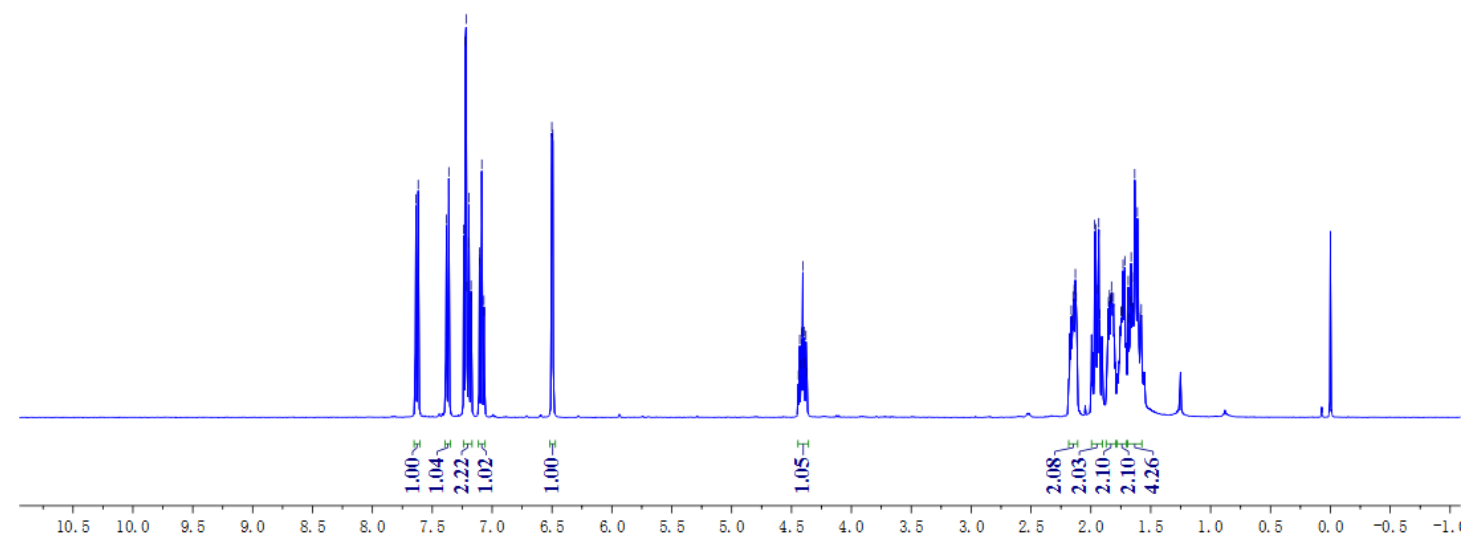

${ }^{13} \mathrm{C}$ NMR spectrum of compound $\mathbf{3 p}\left(\mathrm{CDCl}_{3}, 101 \mathrm{MHz}\right)$
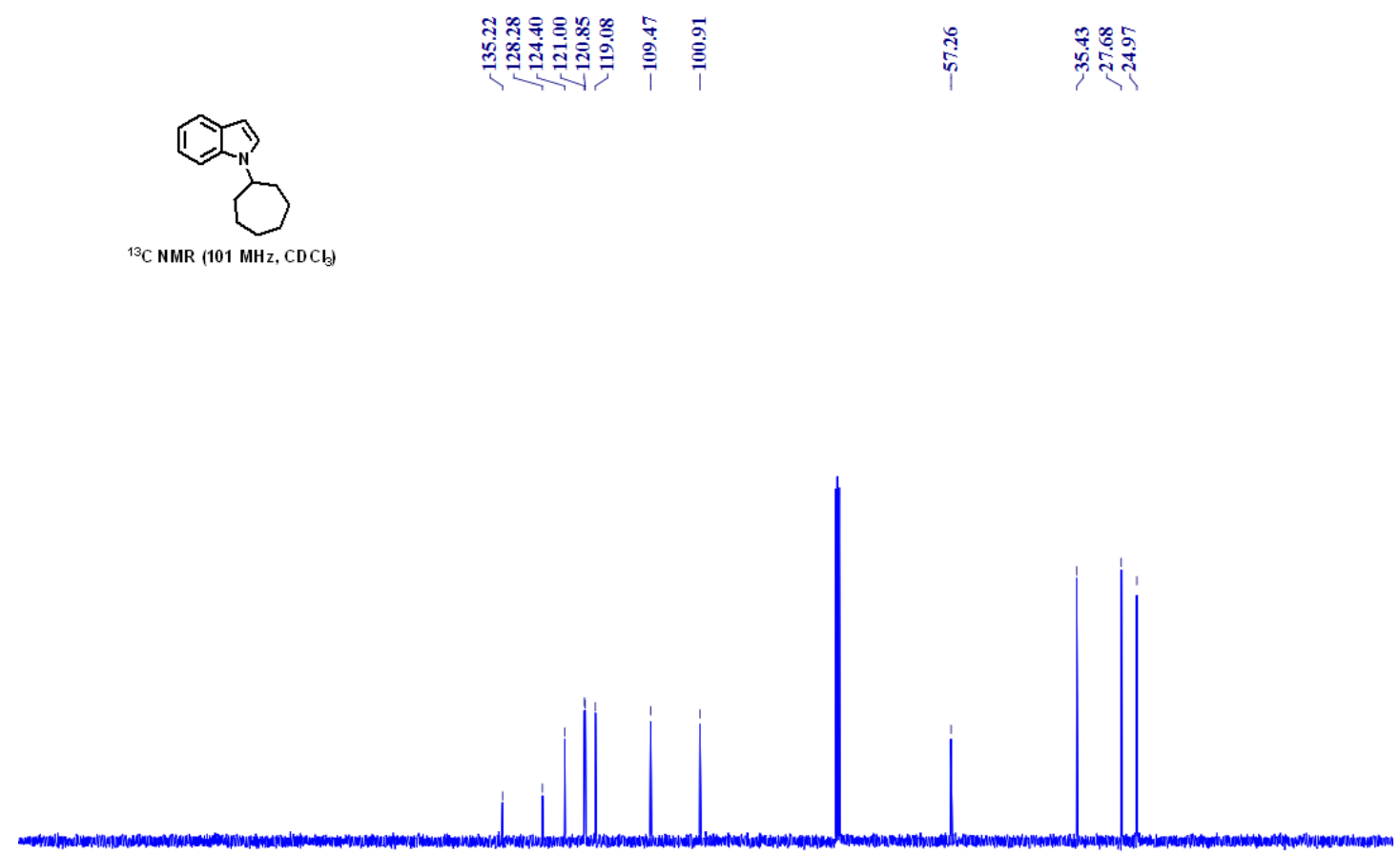

$\begin{array}{lllllllllllllllllllllll}1 & 1 \\ 210 & 200 & 190 & 180 & 170 & 160 & 150 & 140 & 130 & 120 & 110 & 100 & 90 & 80 & 70 & 60 & 50 & 40 & 30 & 20 & 10 & 0 & -10\end{array}$ 
${ }^{1} \mathrm{H}$ NMR spectrum of compound $\mathbf{3 q}$ (major product) $\left(\mathrm{CDCl}_{3}, 400 \mathrm{MHz}\right)$

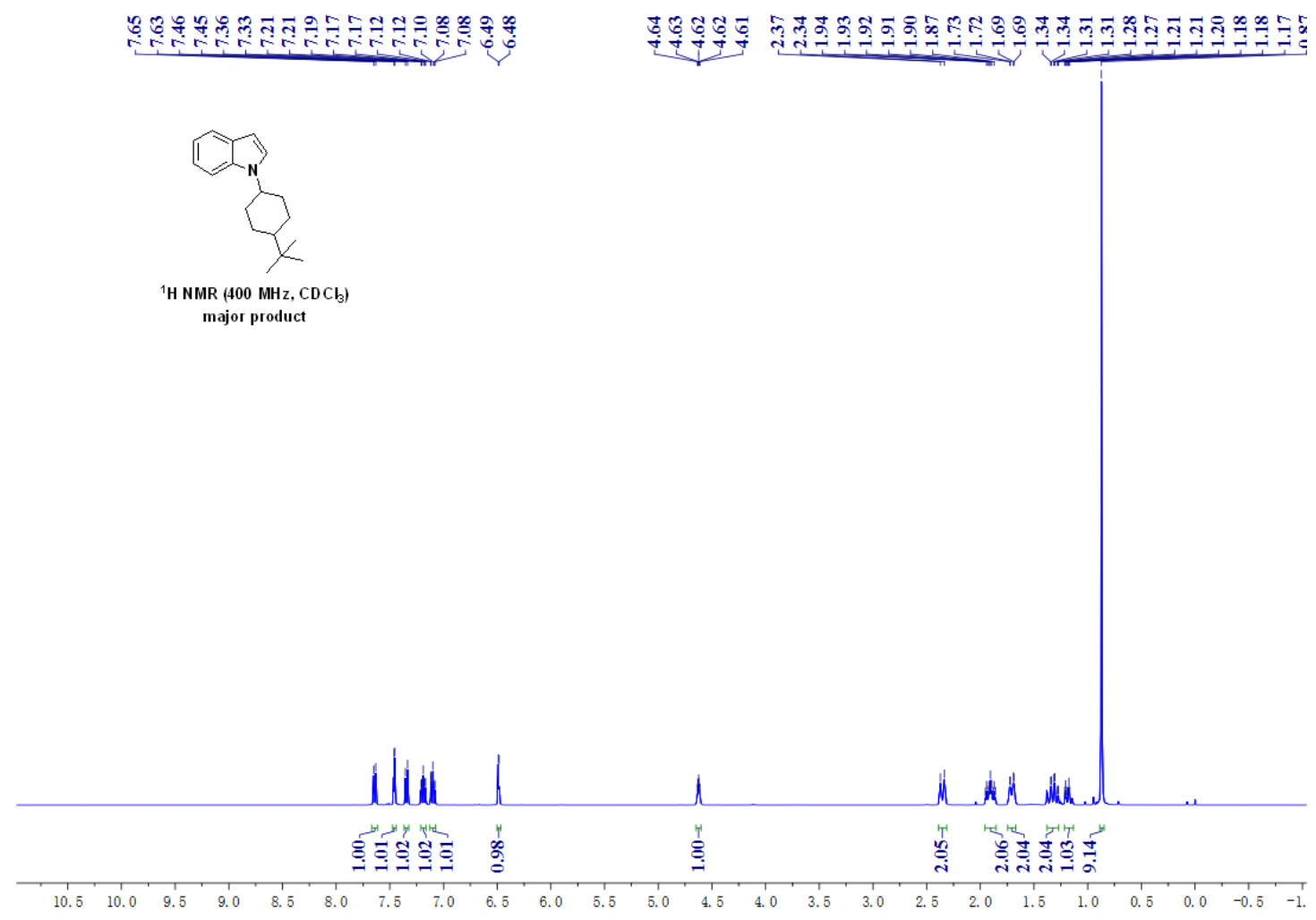

${ }^{13} \mathrm{C}$ NMR spectrum of compound $\mathbf{3 q}$ (major product) $\left(\mathrm{CDCl}_{3}, 101 \mathrm{MHz}\right)$
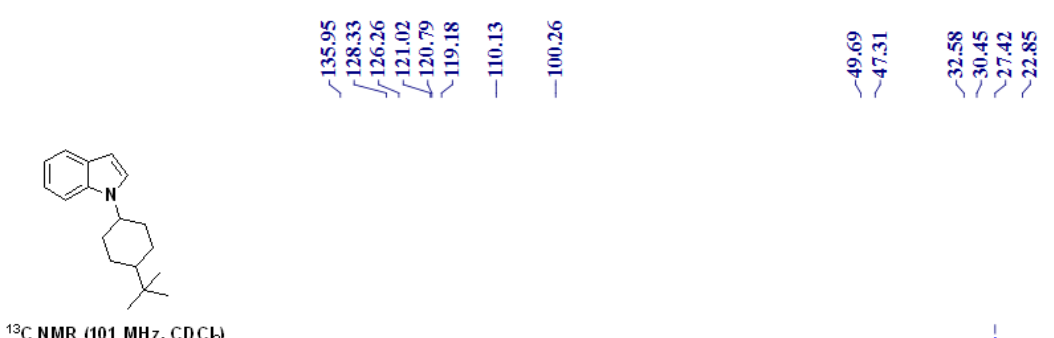

NMR (101 MHz, CDC

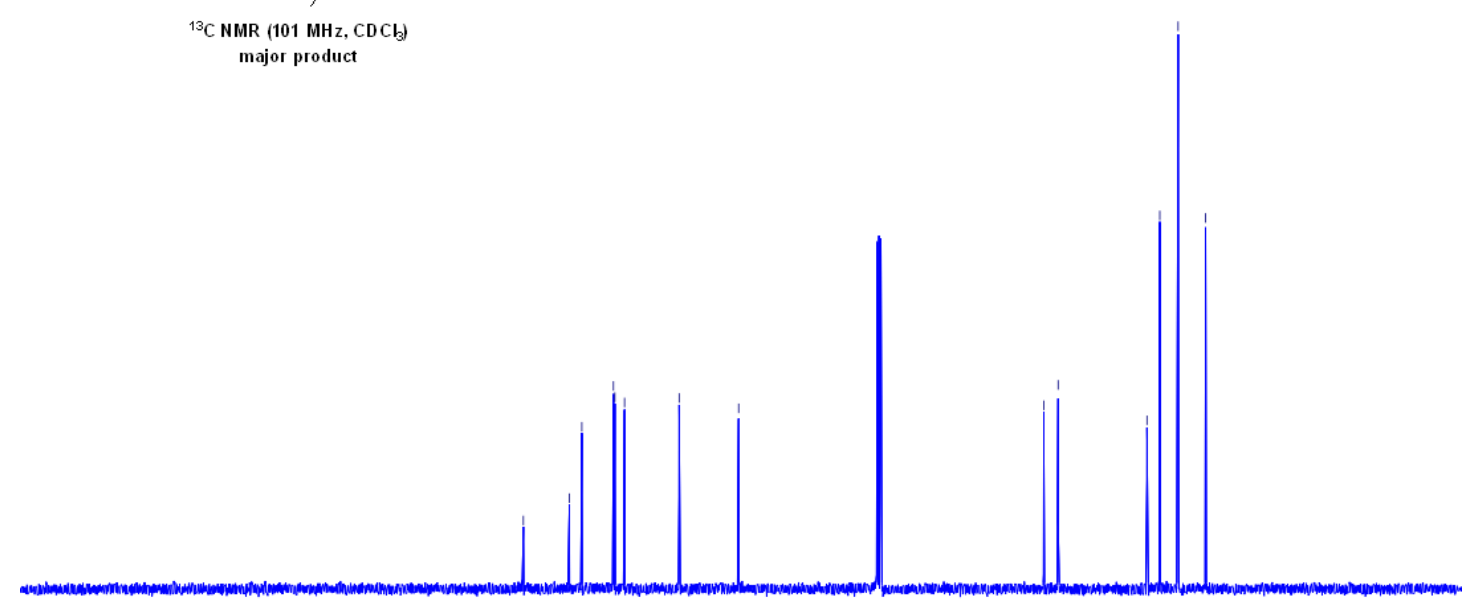

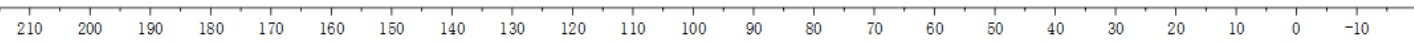


${ }^{1} \mathrm{H}$ NMR spectrum of compound $\mathbf{3 q}$ (minor product) $\left(\mathrm{CDCl}_{3}, 400 \mathrm{MHz}\right)$

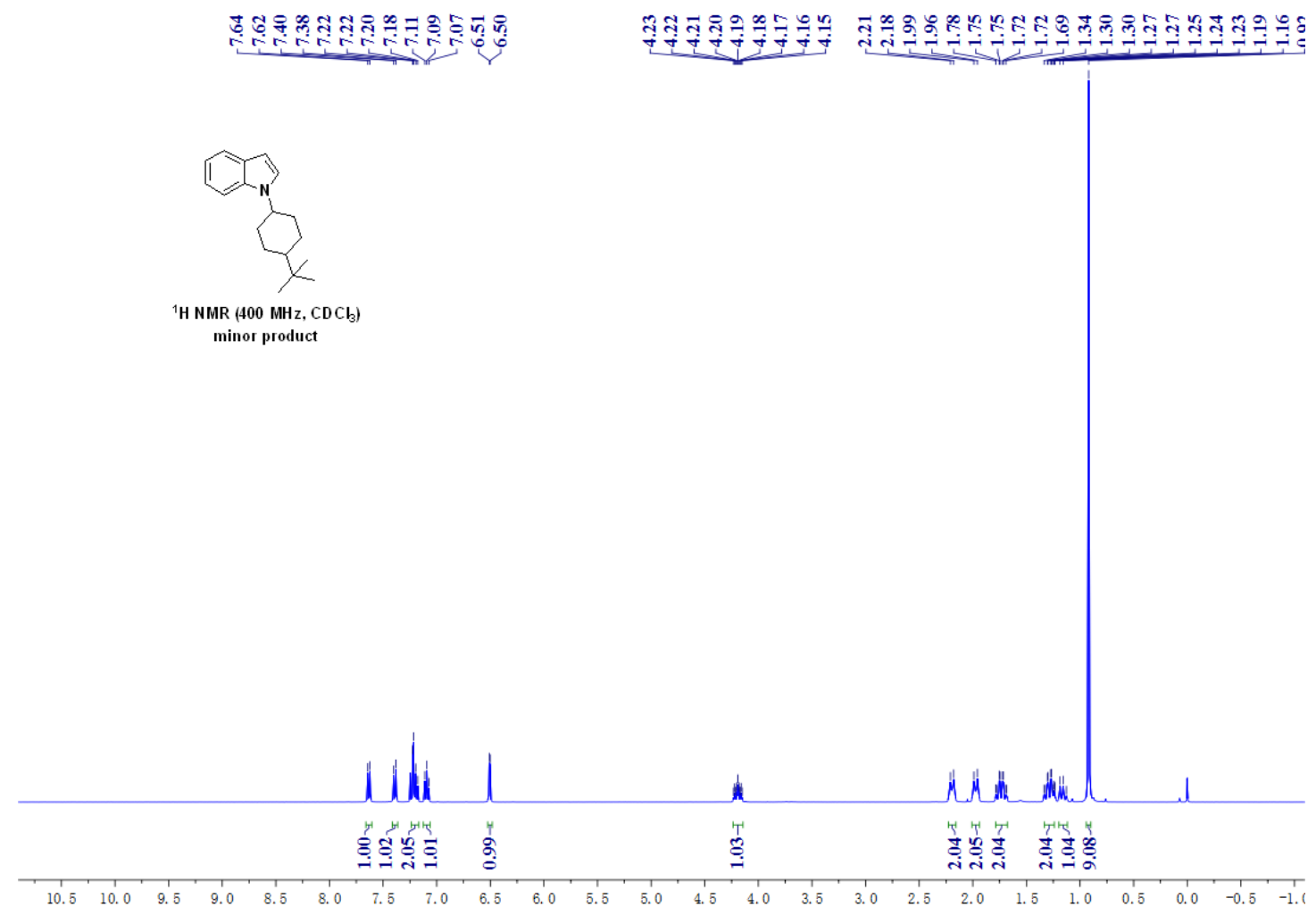

${ }^{13} \mathrm{C}$ NMR spectrum of compound $\mathbf{3 q}$ (minor product) $\left(\mathrm{CDCl}_{3}, 101 \mathrm{MHz}\right)$
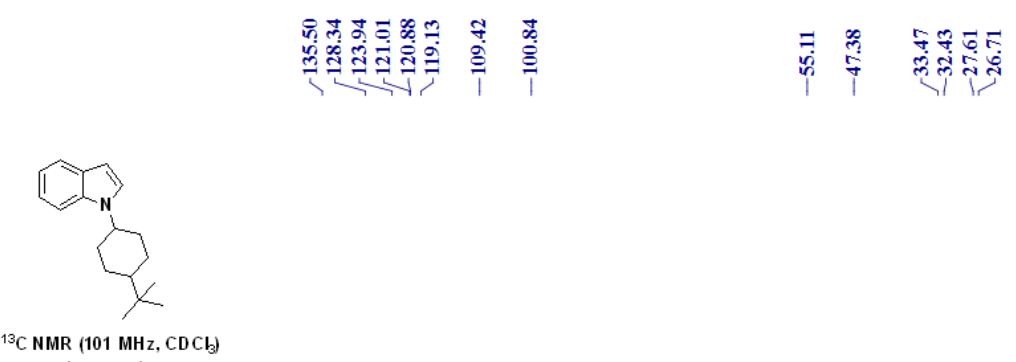

minor product

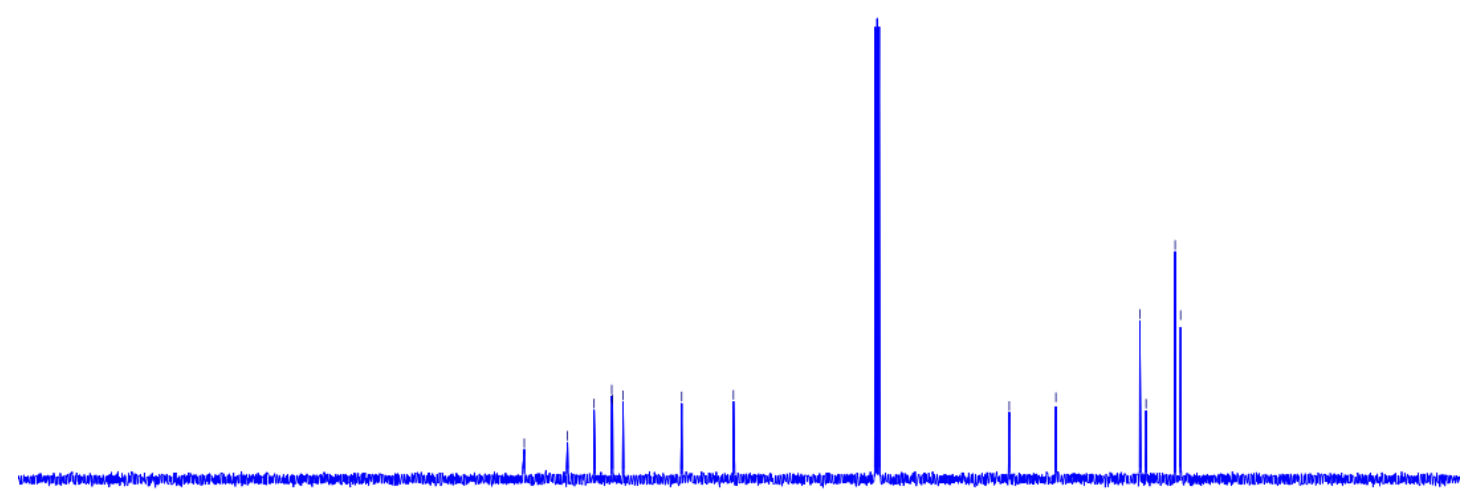

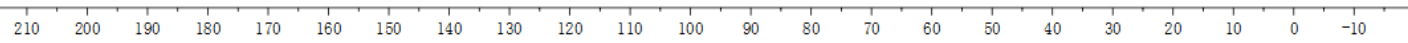


${ }^{1} \mathrm{H}$ NMR spectrum of compound $\mathbf{3 r}\left(\mathrm{CDCl}_{3}, 400 \mathrm{MHz}\right)$

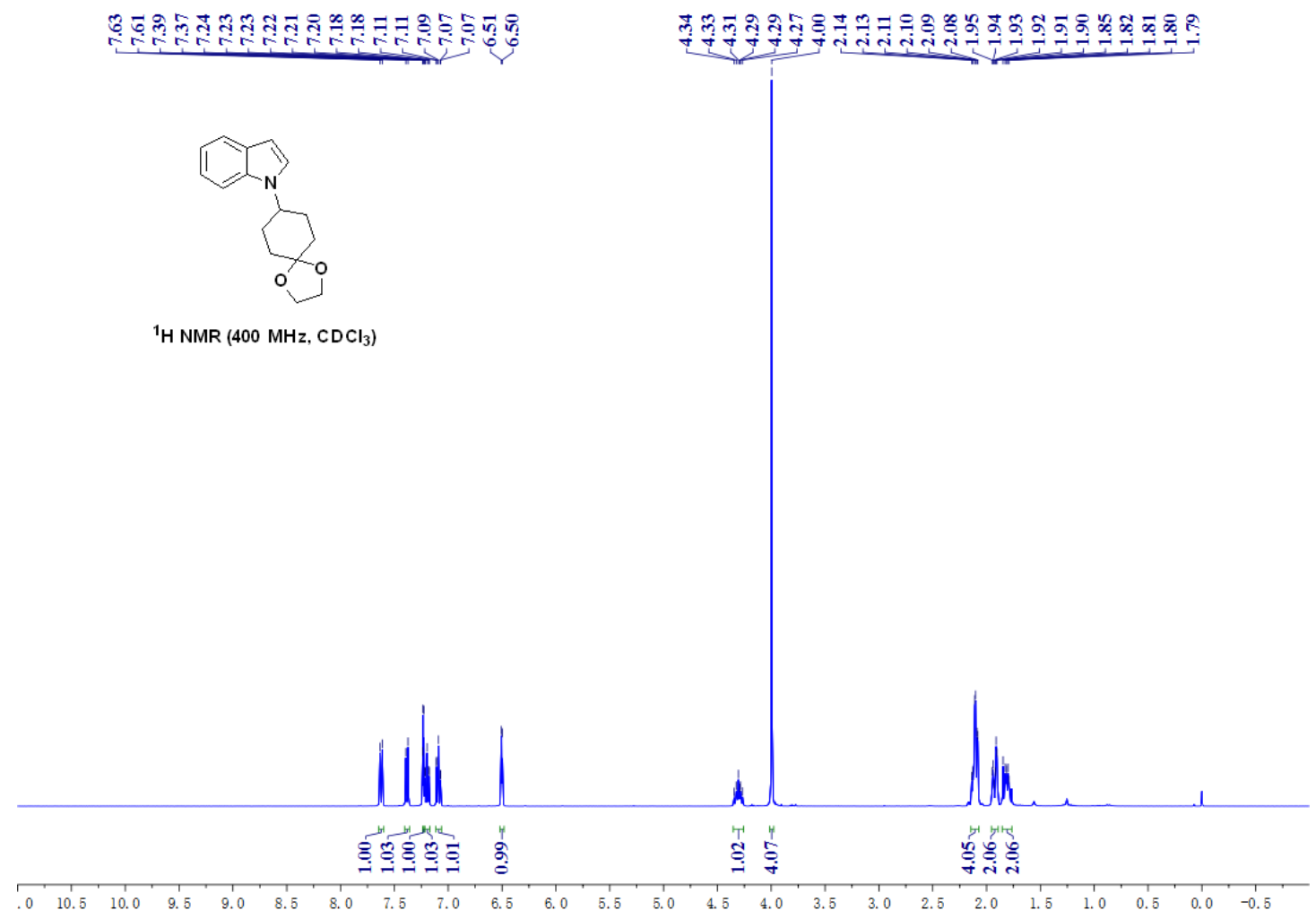

${ }^{13} \mathrm{C}$ NMR spectrum of compound $3 \mathbf{r}\left(\mathrm{CDCl}_{3}, 101 \mathrm{MHz}\right)$
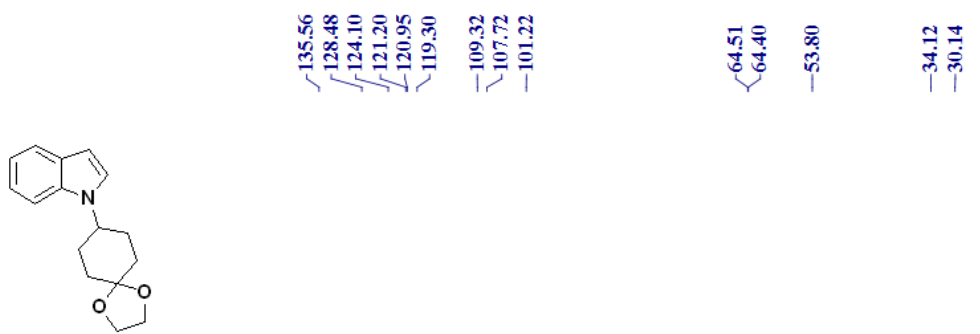

${ }^{13} \mathrm{C}$ NMR (101 MHz, $\left.\mathrm{CDCl}_{3}\right)$

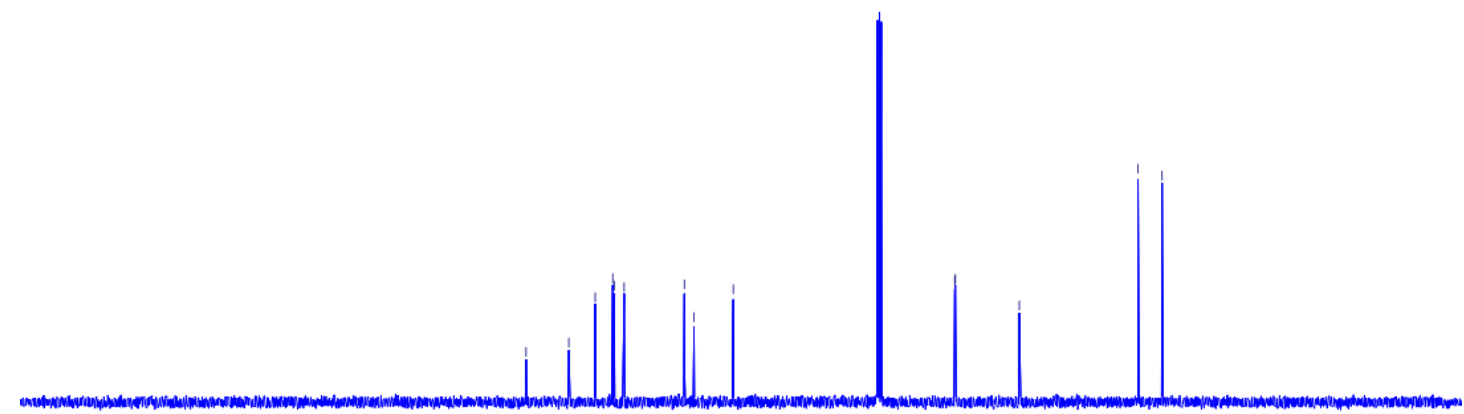

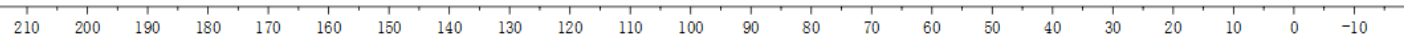


${ }^{1} \mathrm{H}$ NMR spectrum of compound $\mathbf{3 s}\left(\mathrm{CDCl}_{3}, 400 \mathrm{MHz}\right)$
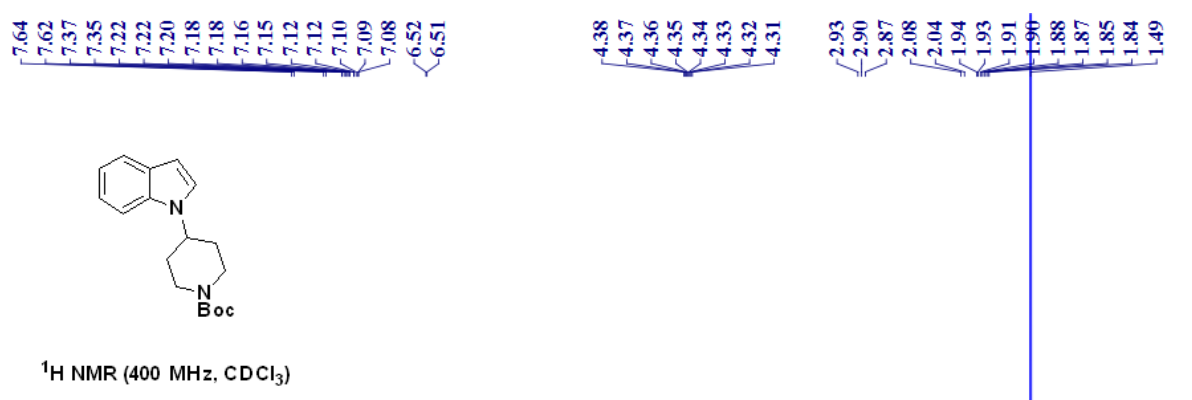

${ }^{1} \mathrm{H}$ NMR $\left(400 \mathrm{MHz}, \mathrm{CDCl}_{3}\right)$

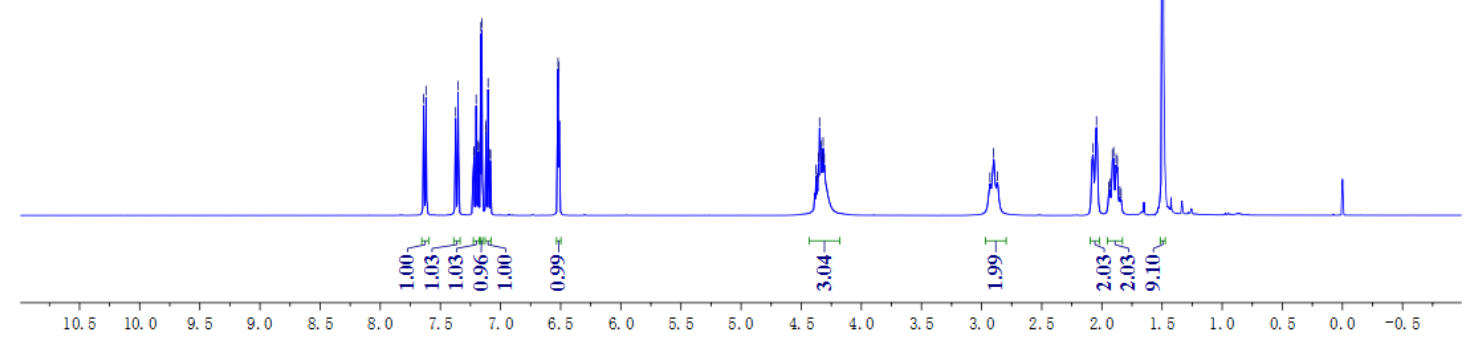

${ }^{13} \mathrm{C}$ NMR spectrum of compound 3 s $\left(\mathrm{CDCl}_{3}, 101 \mathrm{MHz}\right)$
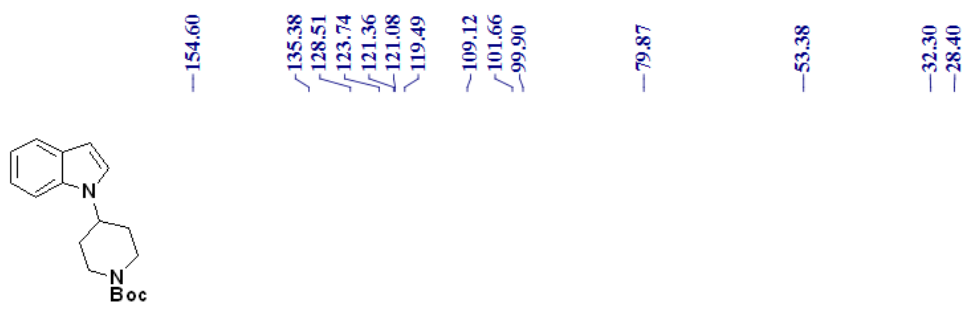

${ }^{13} \mathrm{C}$ NMR (101 MHz, $\mathrm{CDCl}_{3}$ )

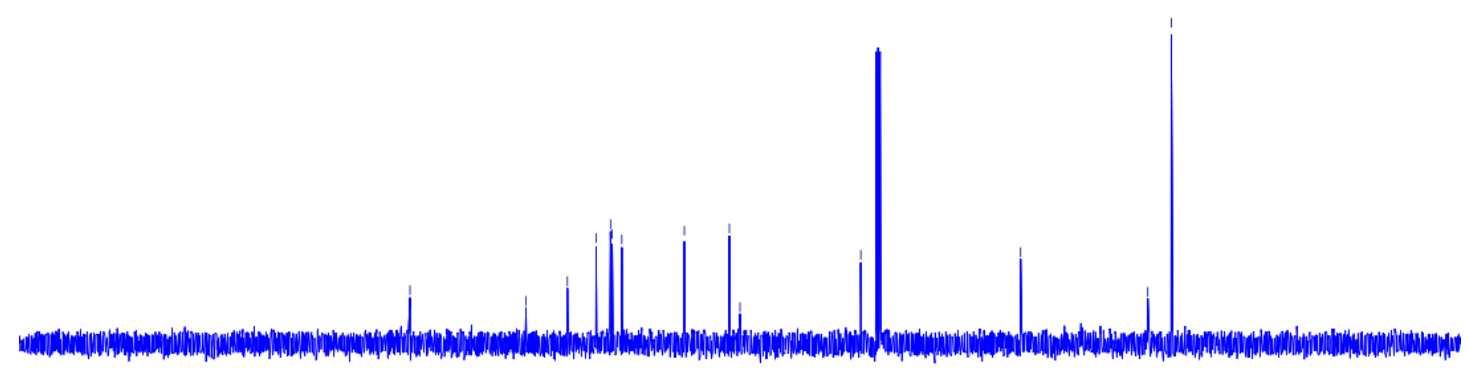

$\begin{array}{llllllll}210 & 200 & 190 & 180 & 170 & 160 & 150\end{array}$

$\begin{array}{lll}130 & 130 & 120\end{array}$

$\begin{array}{lll}110 & 100 & 90\end{array}$ 
${ }^{1} \mathrm{H}$ NMR spectrum of compound $\mathbf{3 t}\left(\mathrm{CDCl}_{3}, 400 \mathrm{MHz}\right)$

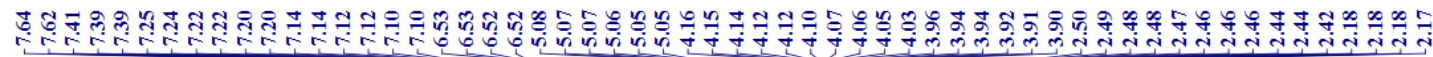

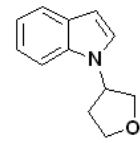

${ }^{1} \mathrm{H}$ NMR $\left(400 \mathrm{MHz}, \mathrm{CDCl}_{3}\right)$

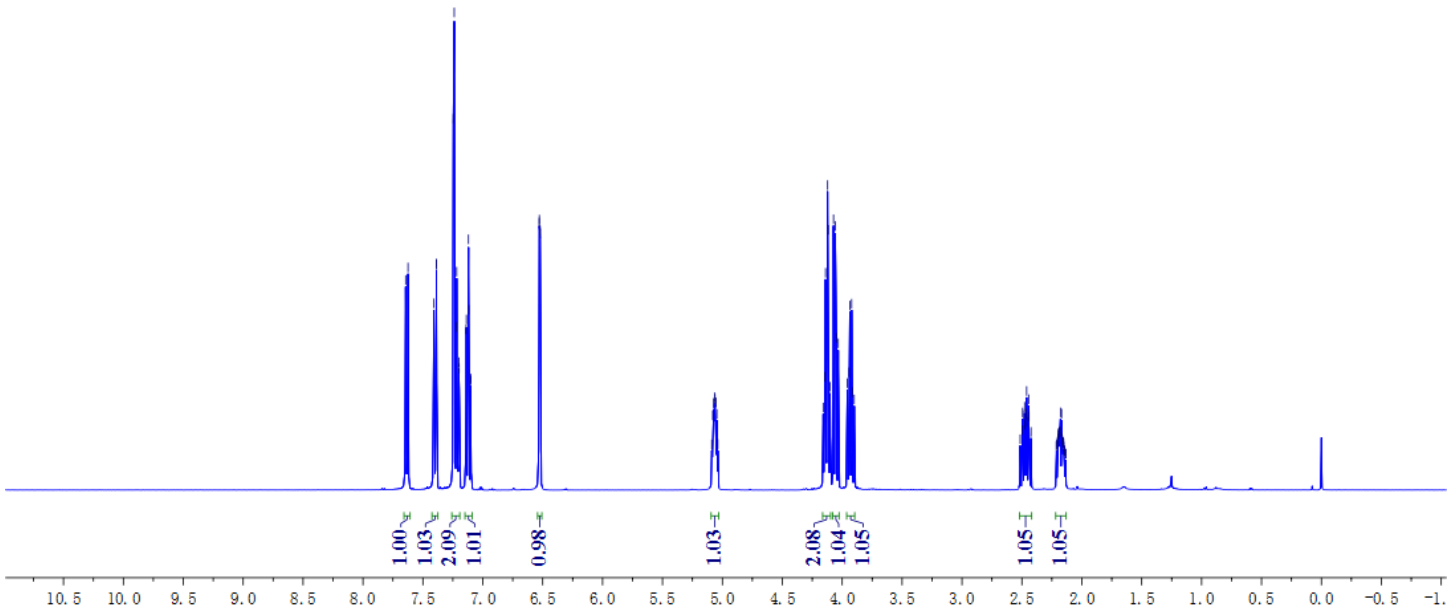

${ }^{13} \mathrm{C}$ NMR spectrum of compound $3 \mathrm{t}\left(\mathrm{CDCl}_{3}, 101 \mathrm{MHz}\right)$

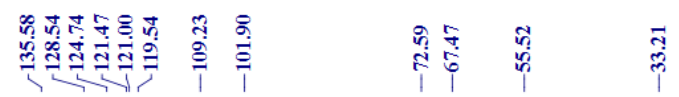

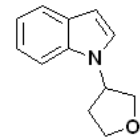

${ }^{13} \mathrm{C}$ NMR $\left(101 \mathrm{MHz}, \mathrm{CDCl}_{3}\right)$

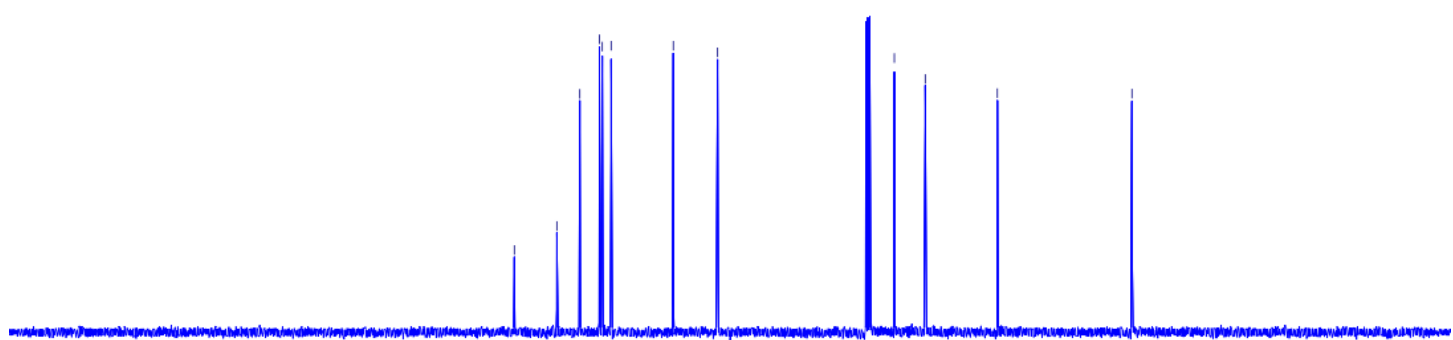

$\begin{array}{lllllllllllllllllllllll}1 & 1 \\ 210 & 200 & 190 & 180 & 170 & 160 & 150 & 140 & 130 & 120 & 110 & 100 & 90 & 80 & 70 & 60 & 50 & 40 & 30 & 20 & 10 & 0 & -10\end{array}$ 
${ }^{1} \mathrm{H}$ NMR spectrum of compound $\mathbf{3} \mathbf{u}\left(\mathrm{CDCl}_{3}, 400 \mathrm{MHz}\right)$

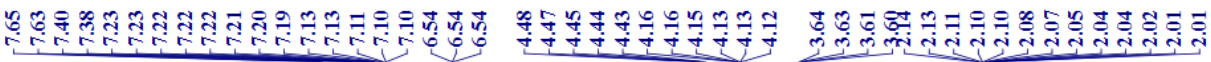

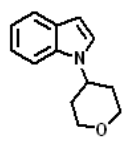

${ }^{1} \mathrm{H}$ NMR $\left(400 \mathrm{MHz}, \mathrm{CDCl}_{3}\right)$

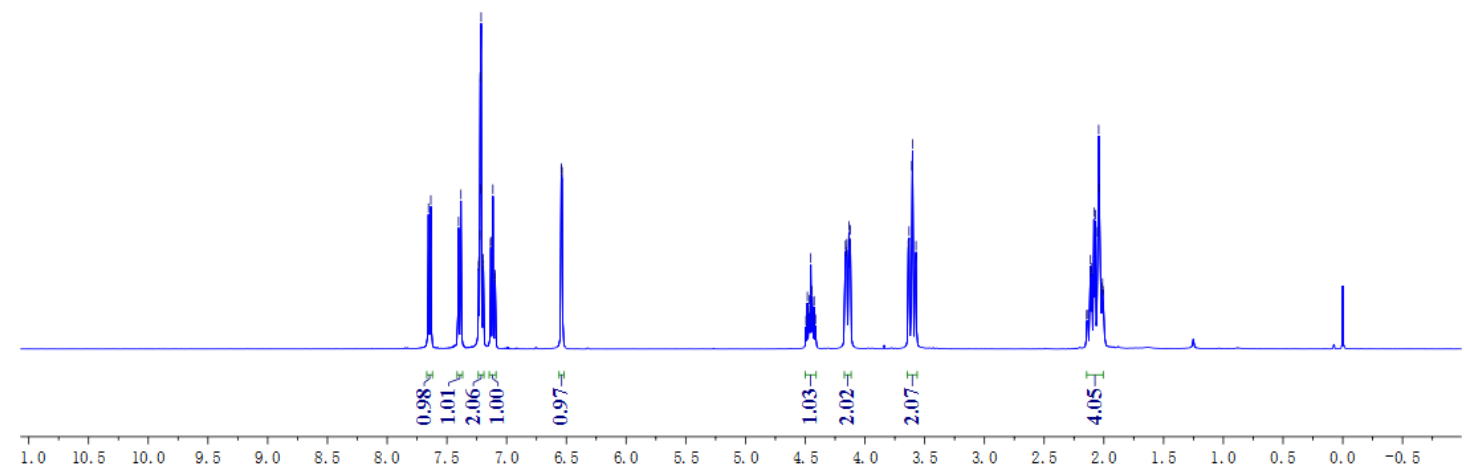

${ }^{13} \mathrm{C}$ NMR spectrum of compound $\mathbf{3 u}\left(\mathrm{CDCl}_{3}, 101 \mathrm{MHz}\right)$
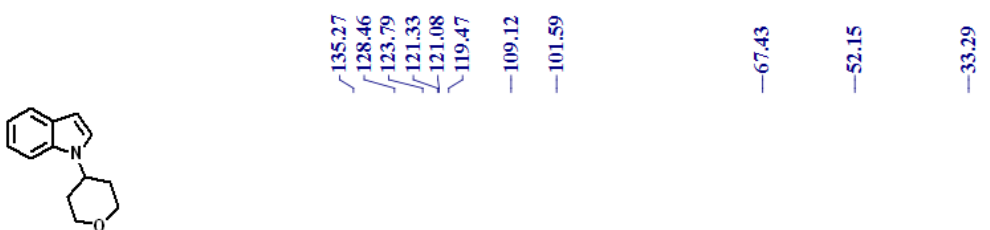

${ }^{13} \mathrm{C}$ NMR (101 MHz, $\mathrm{CDCl}$ )

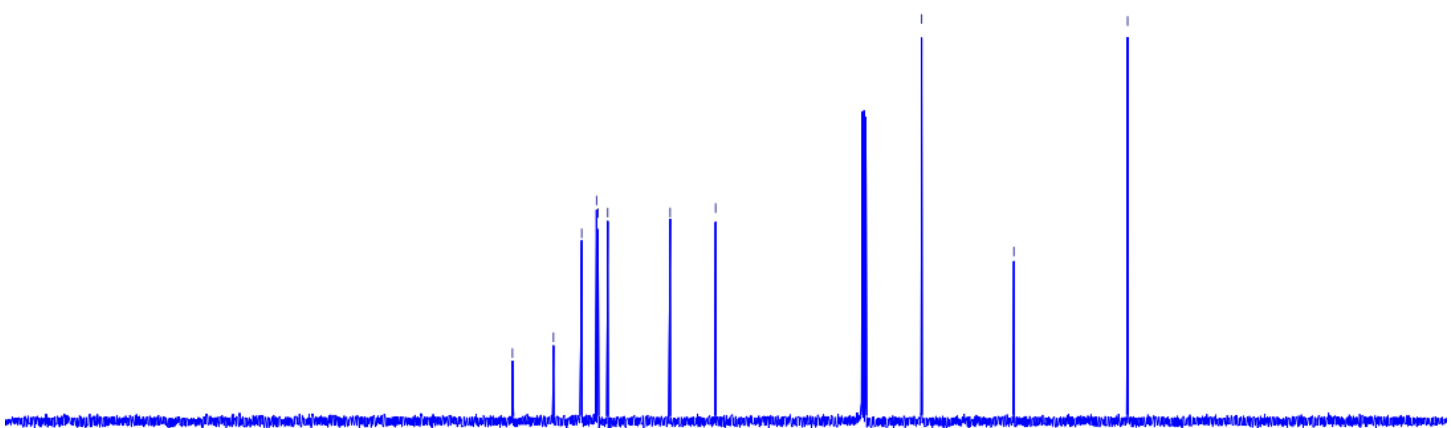

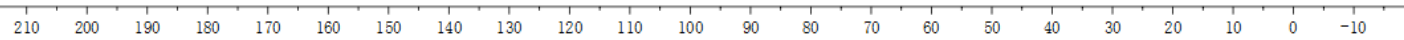


${ }^{1} \mathrm{H}$ NMR spectrum of compound $\mathbf{3 v}\left(\mathrm{CDCl}_{3}, 600 \mathrm{MHz}\right)$
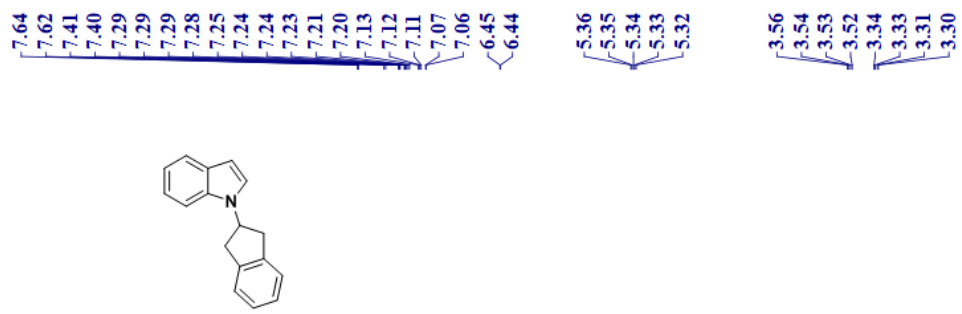

${ }^{1} \mathrm{H} \mathrm{NMR}\left(600 \mathrm{MHz}, \mathrm{CDCl}_{3}\right)$

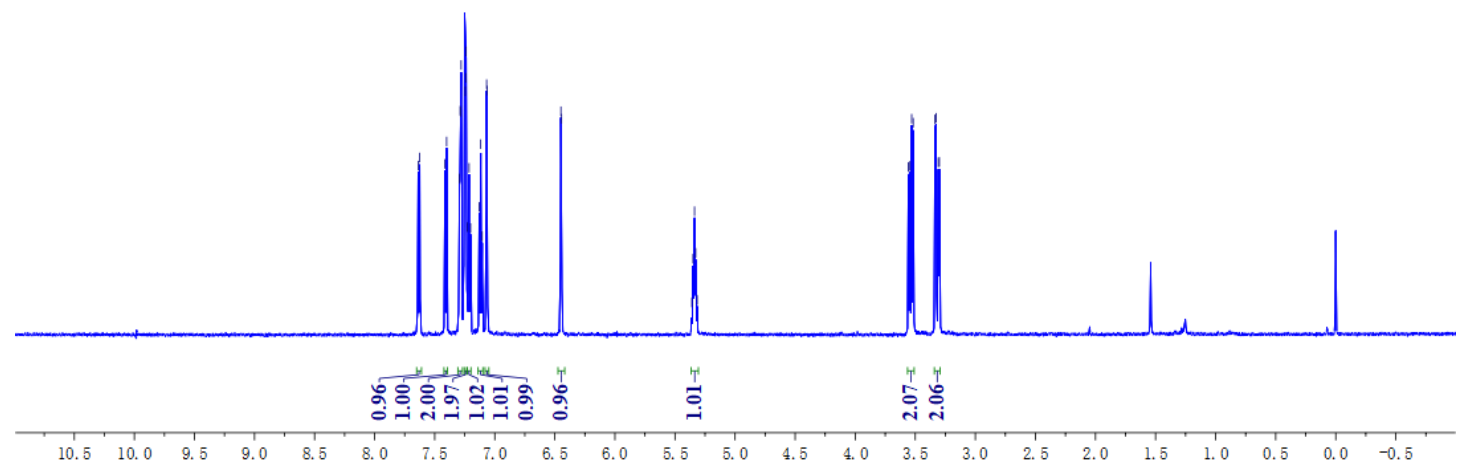

${ }^{13} \mathrm{C}$ NMR spectrum of compound $\mathbf{3 v}\left(\mathrm{CDCl}_{3}, 151 \mathrm{MHz}\right)$
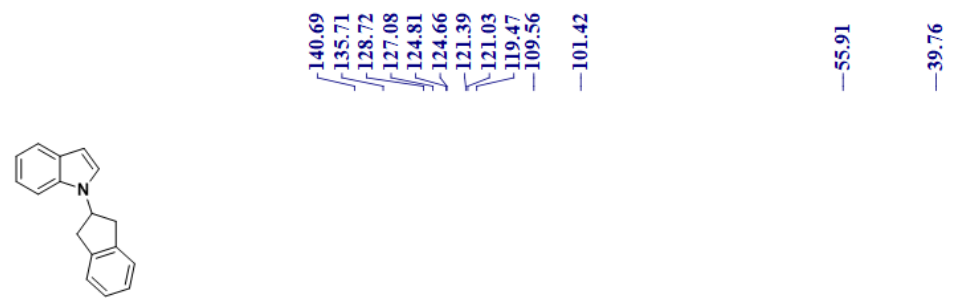

${ }^{13} \mathrm{C}$ NMR (151 MHz, $\left.\mathrm{CDCl}_{3}\right)$

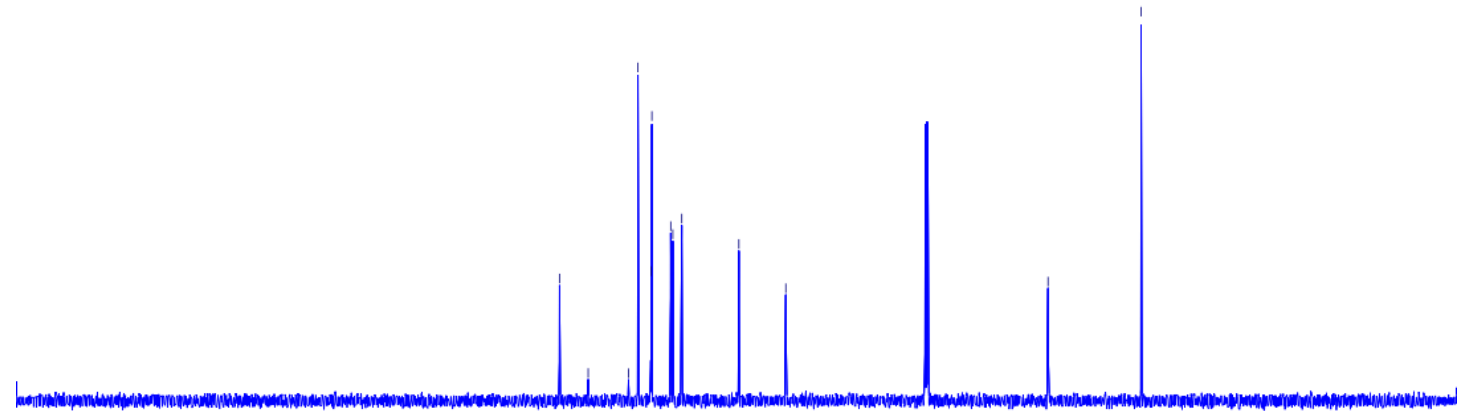

${ }_{230}^{1} \quad \begin{array}{lllllllllllllllllllllllll}1 & 220 & 210 & 200 & 190 & 180 & 170 & 160 & 150 & 140 & 130 & 120 & 110 & 100 & 90 & 80 & 70 & 60 & 50 & 40 & 30 & 20 & 10 & 0 & -10\end{array}$ 
${ }^{1} \mathrm{H}$ NMR spectrum of compound $\mathbf{3 w}\left(\mathrm{CDCl}_{3}, 400 \mathrm{MHz}\right)$

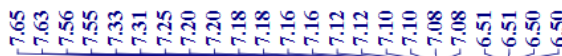

$\stackrel{+\vec{t}}{+}$

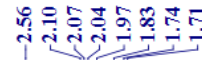

$\mathbb{N}_{\mathrm{N}}$

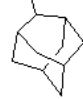

${ }^{1} \mathrm{H}$ NMR $\left(400 \mathrm{MHz}^{-\mathrm{CDCl}_{3}}\right)$

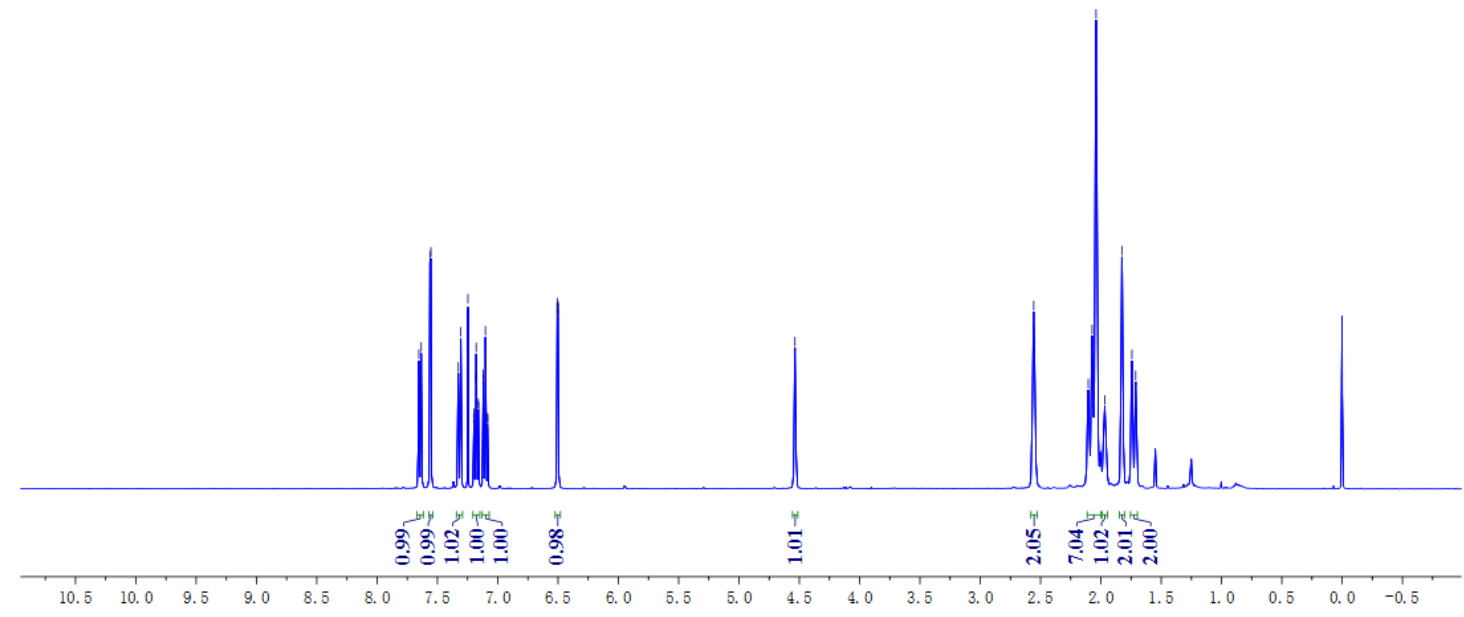

${ }^{13} \mathrm{C}$ NMR spectrum of compound $\mathbf{3 w}\left(\mathrm{CDCl}_{3}, 101 \mathrm{MHz}\right)$
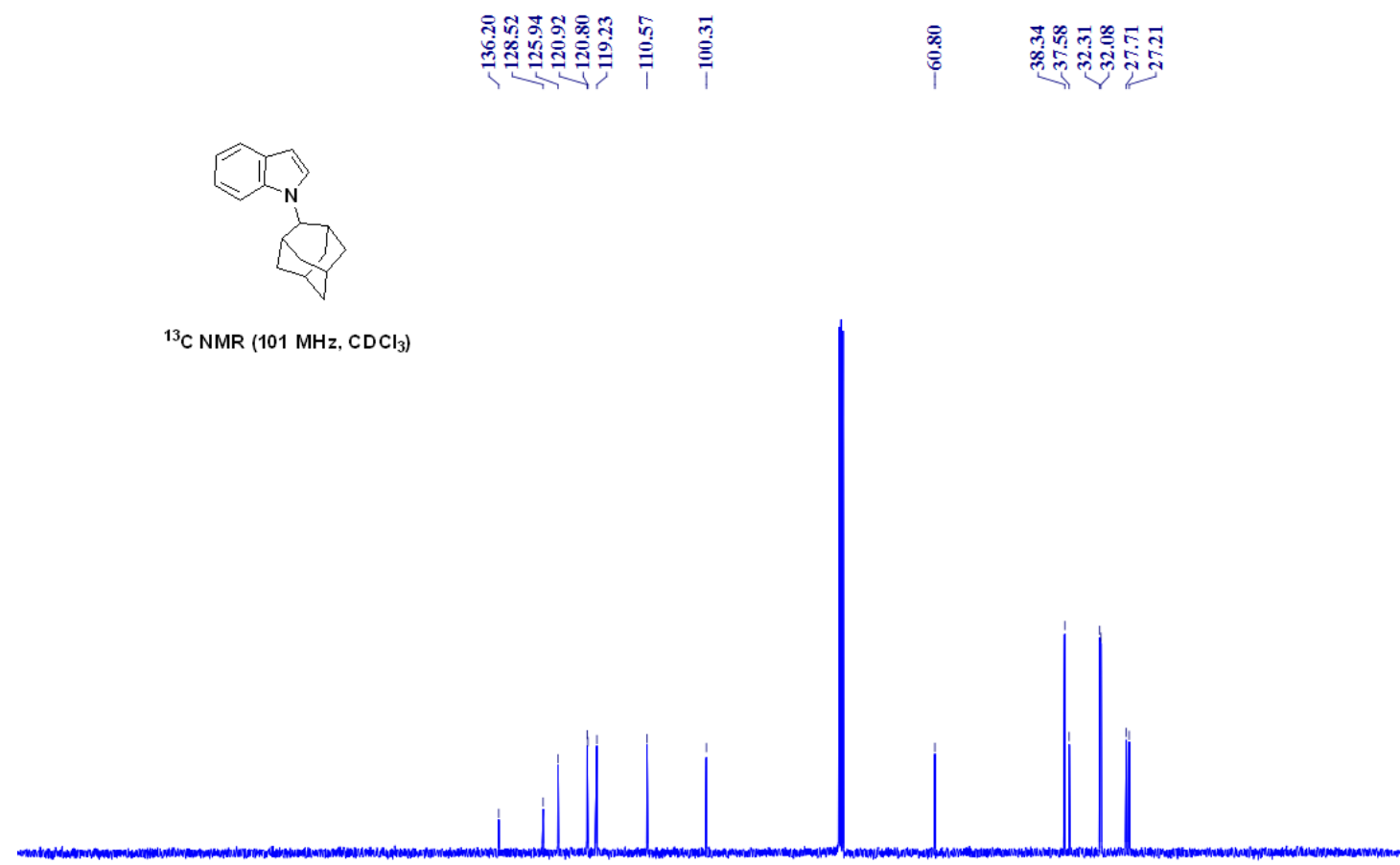

${ }^{13} \mathrm{C}$ NMR $\left(101 \mathrm{MHz}, \mathrm{CDCl}_{3}\right)$

$\begin{array}{lllllll}210 & 200 & 190 & 180 & 170 & 160 & 150\end{array}$
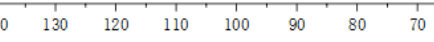
${ }^{1} \mathrm{H}$ NMR spectrum of compound $\mathbf{3 x}\left(\mathrm{CDCl}_{3}, 600 \mathrm{MHz}\right)$
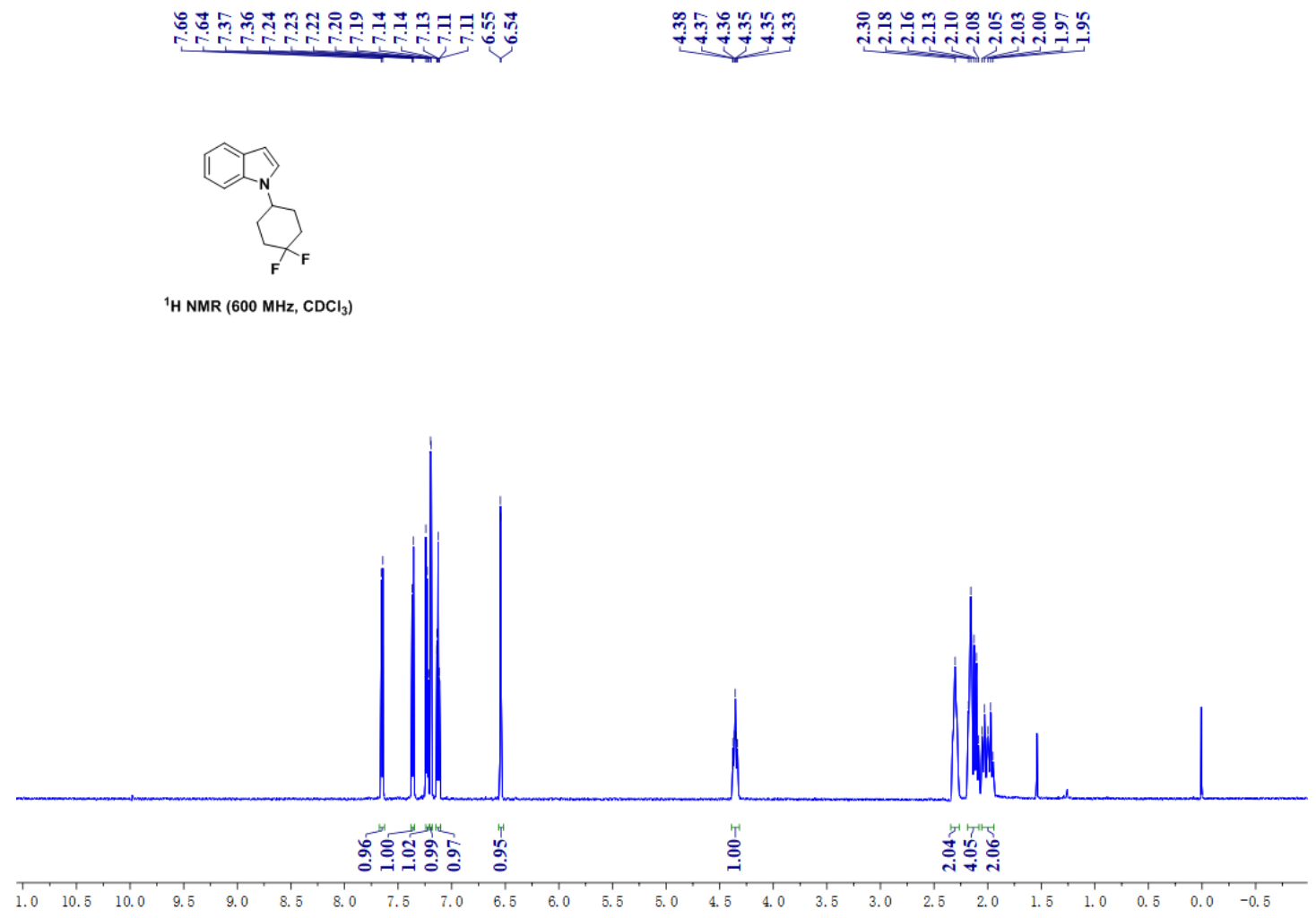

${ }^{13} \mathrm{C} \mathrm{NMR}$ spectrum of compound $\mathbf{3} \mathbf{x}\left(\mathrm{CDCl}_{3}, 151 \mathrm{MHz}\right)$
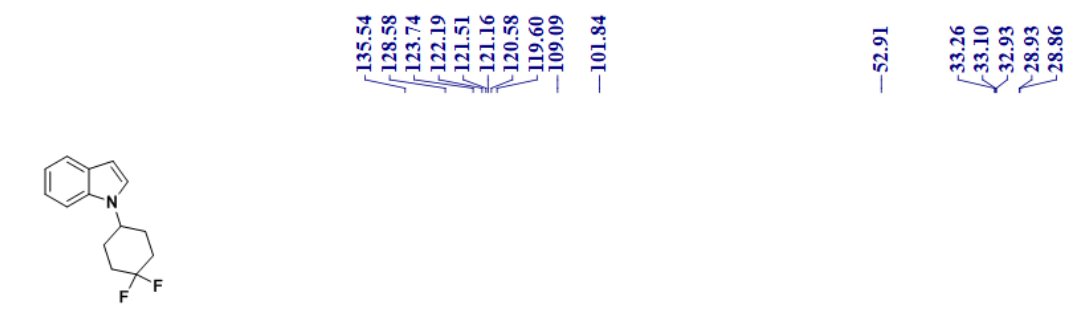

${ }^{13} \mathrm{C} \mathrm{NMR}\left(151 \mathrm{MHz}, \mathrm{CDCl}_{3}\right)$

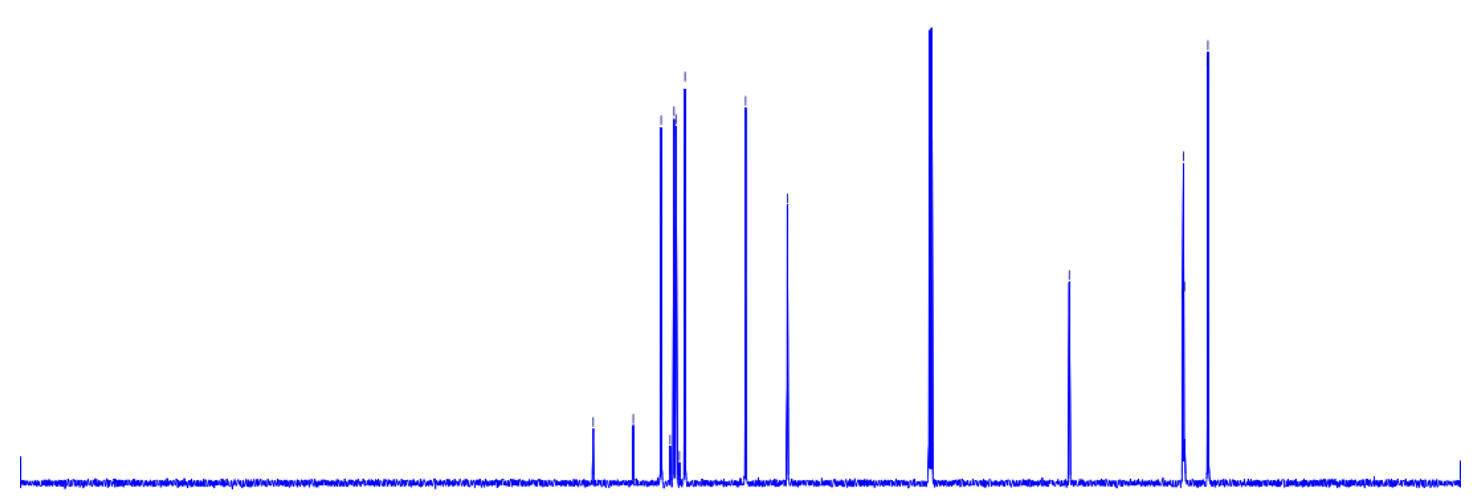

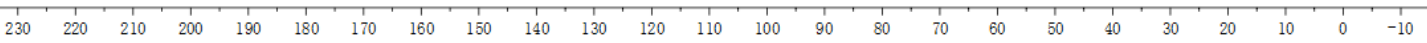


${ }^{19} \mathrm{~F}$ NMR spectrum of compound $\mathbf{3 x}\left(\mathrm{CDCl}_{3}, 376 \mathrm{MHz}\right)$

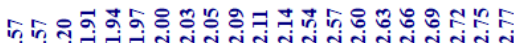

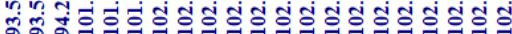

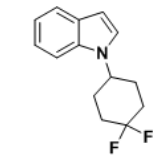

${ }^{19} \mathrm{~F} \mathrm{NMR}\left(376 \mathrm{MHz}, \mathrm{CDCl}_{3}\right)$

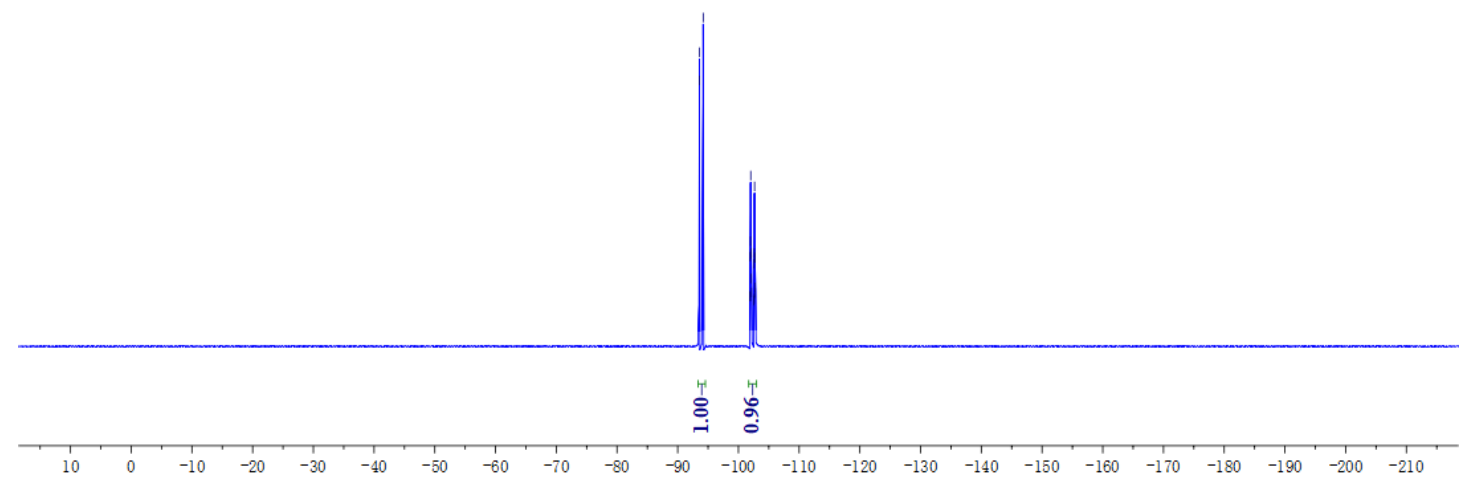

${ }^{1} \mathrm{H}$ NMR spectrum of compound $\mathbf{3 y}\left(\mathrm{CDCl}_{3}, 400 \mathrm{MHz}\right)$

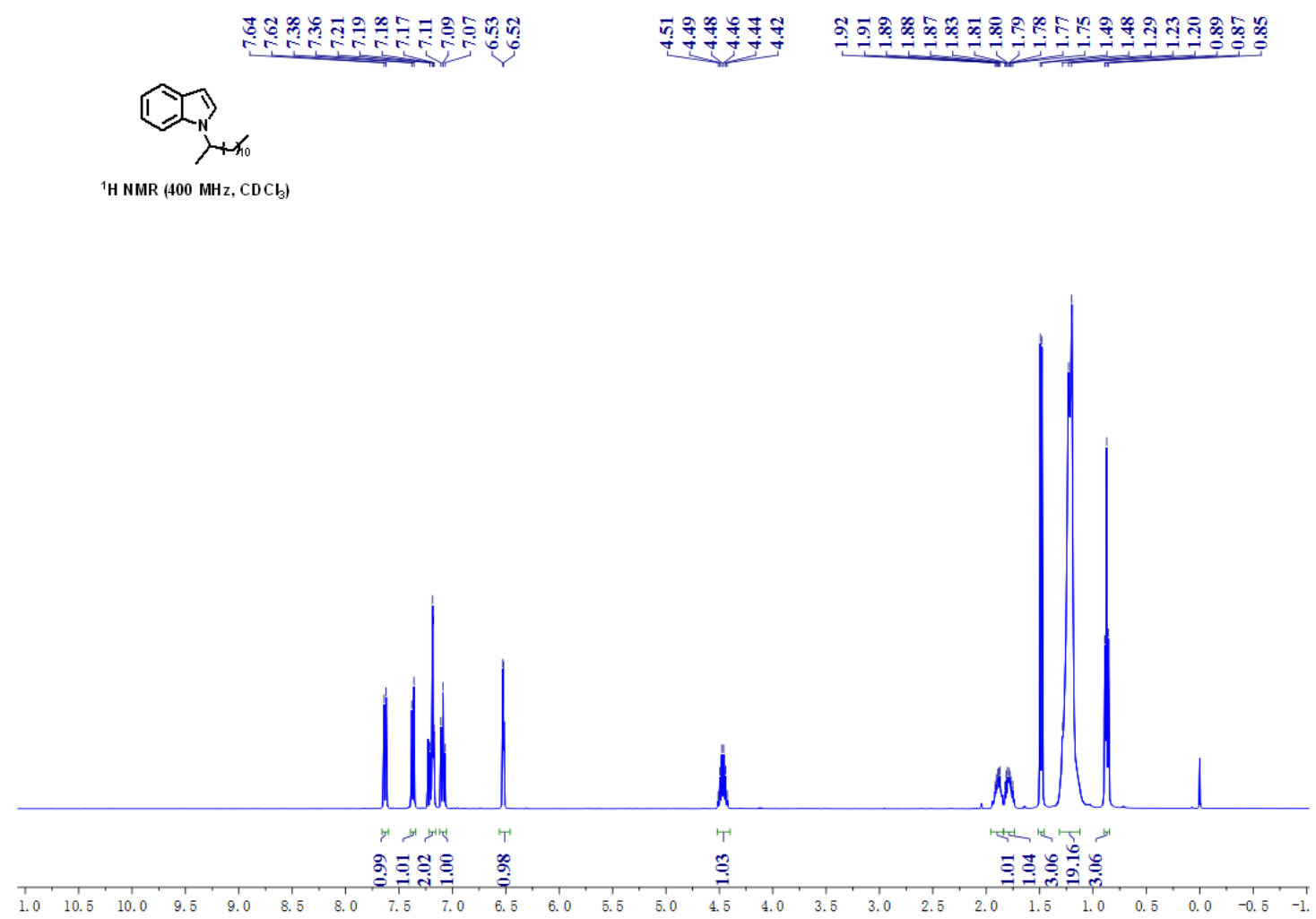


${ }^{13} \mathrm{C} \mathrm{NMR}$ spectrum of compound $\mathbf{3 y}\left(\mathrm{CDCl}_{3}, 101 \mathrm{MHz}\right)$
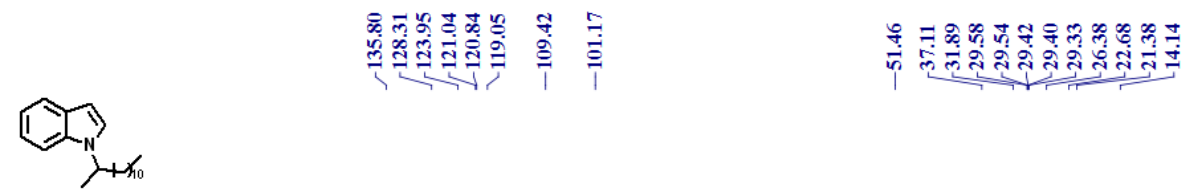

${ }^{13} \mathrm{C}$ NMR $(101 \mathrm{MHz}, \mathrm{CDCl})$

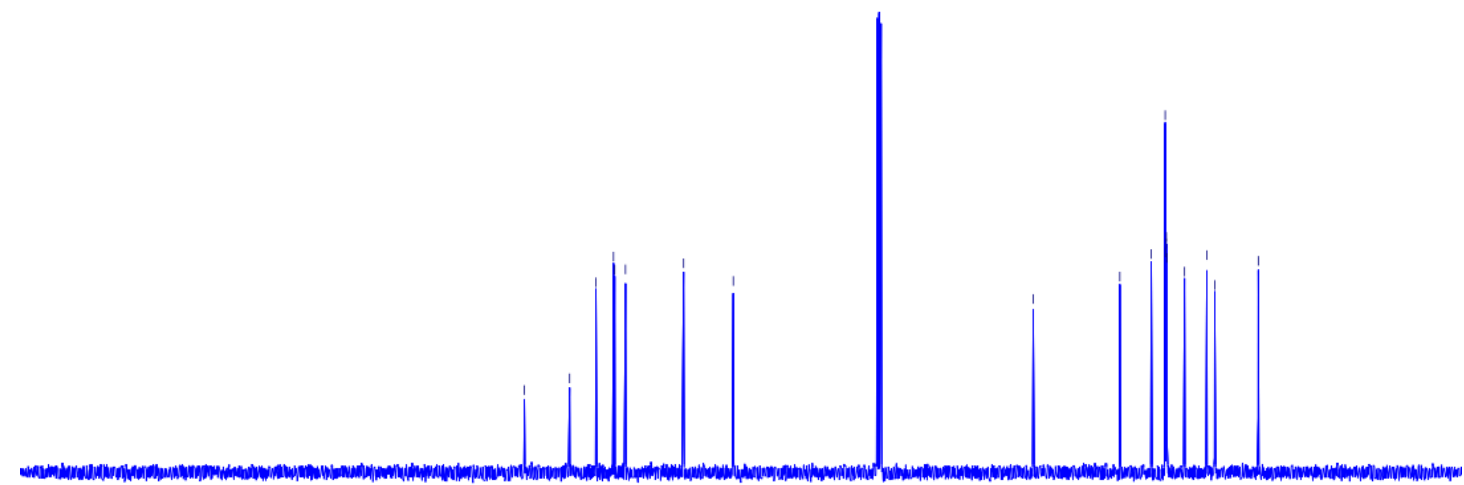

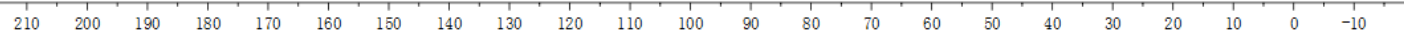

${ }^{1} \mathrm{H}$ NMR spectrum of compound $\mathbf{3 z}\left(\mathrm{CDCl}_{3}, 400 \mathrm{MHz}\right)$

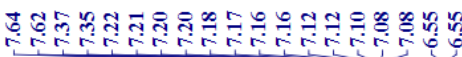

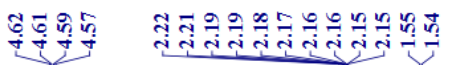

10

$\sim \mathrm{COOH}$

${ }^{1} \mathrm{H}$ NMR $\left(400 \mathrm{MHz}, \mathrm{CDCl}_{3}\right)$

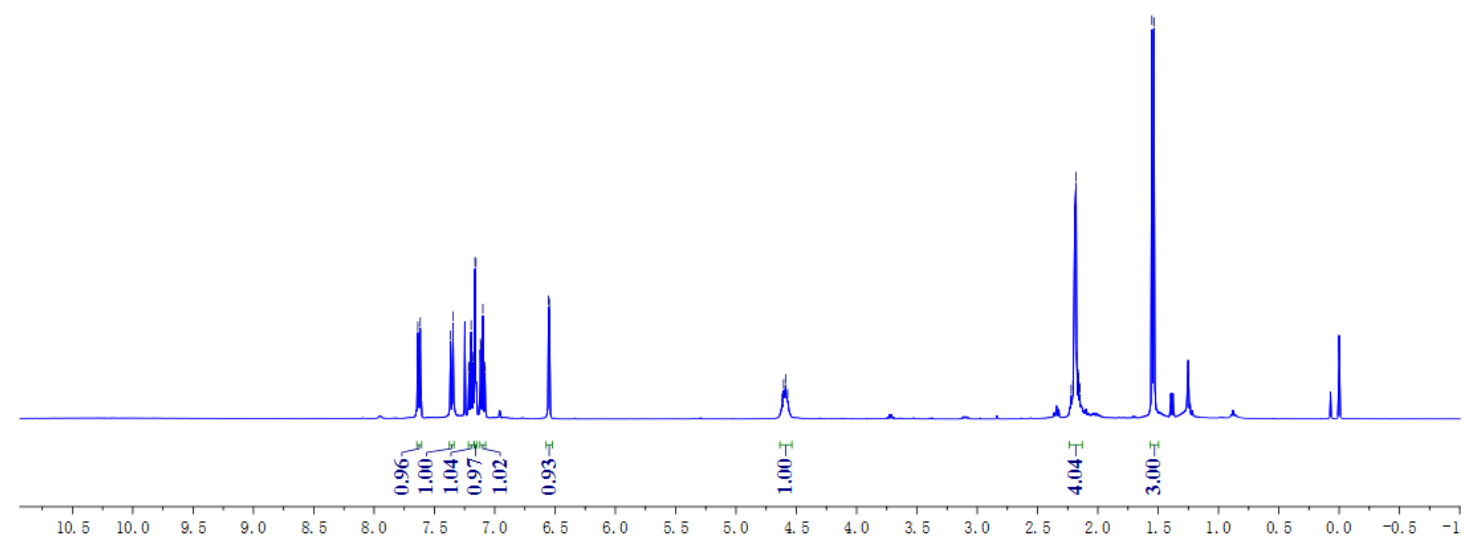


${ }^{13} \mathrm{C}$ NMR spectrum of compound $\mathbf{3 z}\left(\mathrm{CDCl}_{3}, 101 \mathrm{MHz}\right)$

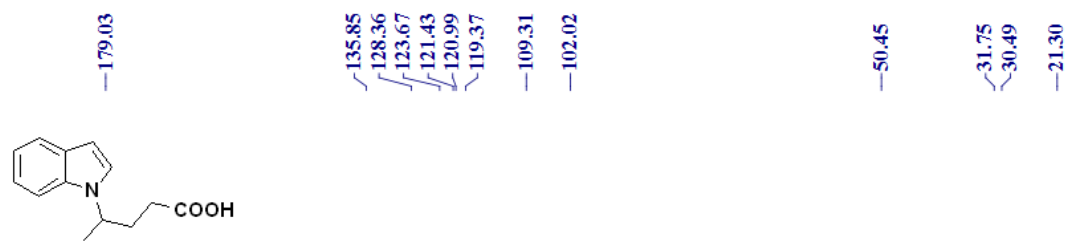

${ }^{13} \mathrm{C}$ NMR $\left(101 \mathrm{MHz}, \mathrm{CDCl}_{3}\right)$
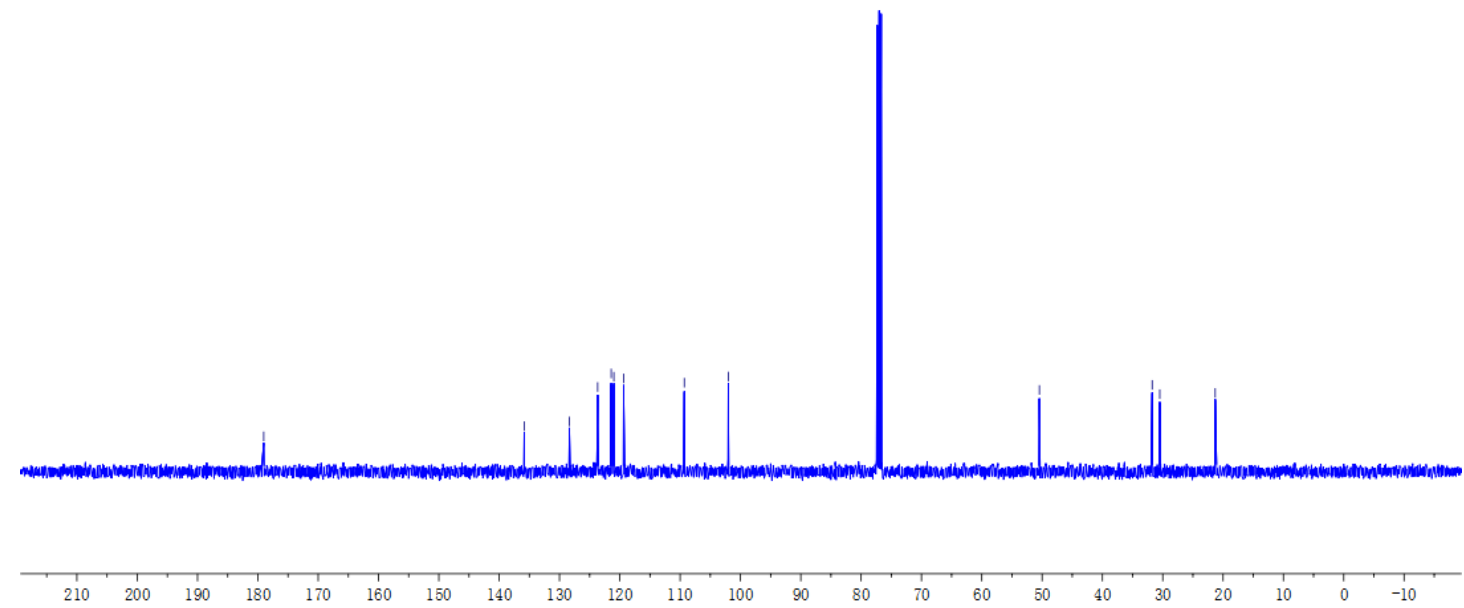

${ }^{1} \mathrm{H}$ NMR spectrum of compound $\mathbf{3 a a}\left(\mathrm{CDCl}_{3}, 400 \mathrm{MHz}\right)$

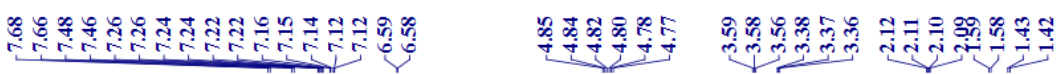<smiles>CC(CCO)n1ccc2ccccc21</smiles>

${ }^{1} \mathrm{H} \mathrm{NMR}\left(400 \mathrm{MHz}, \mathrm{CDCl}_{3}\right)$

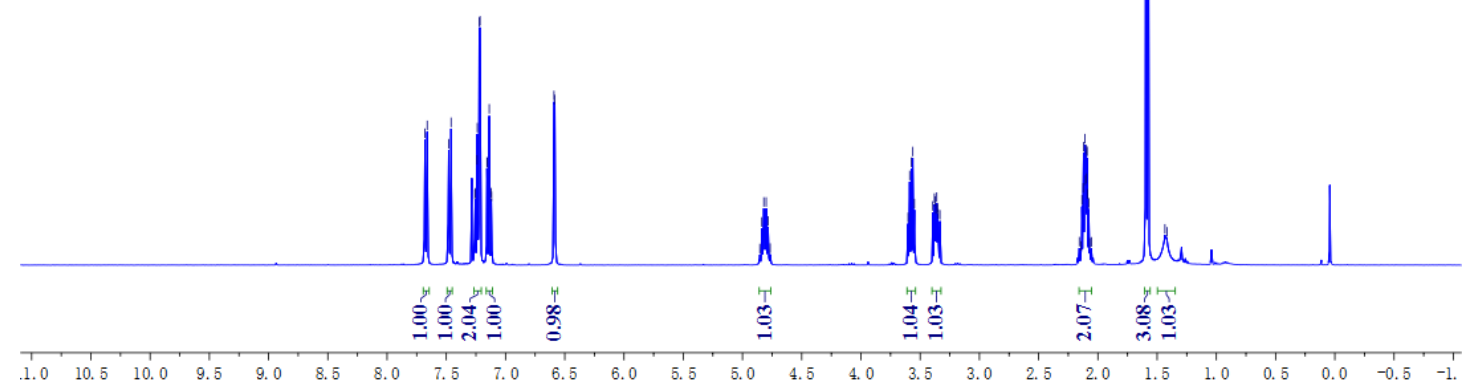


${ }^{13} \mathrm{C}$ NMR spectrum of compound $\mathbf{3 a a}\left(\mathrm{CDCl}_{3}, 101 \mathrm{MHz}\right)$

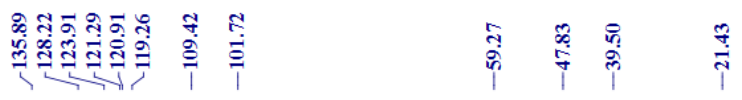

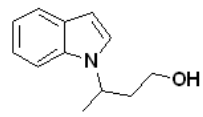

$\left.{ }^{13} \mathrm{C} \mathrm{NMR} \mathrm{(101} \mathrm{MHz}, \mathrm{CDCl}_{3}\right)$

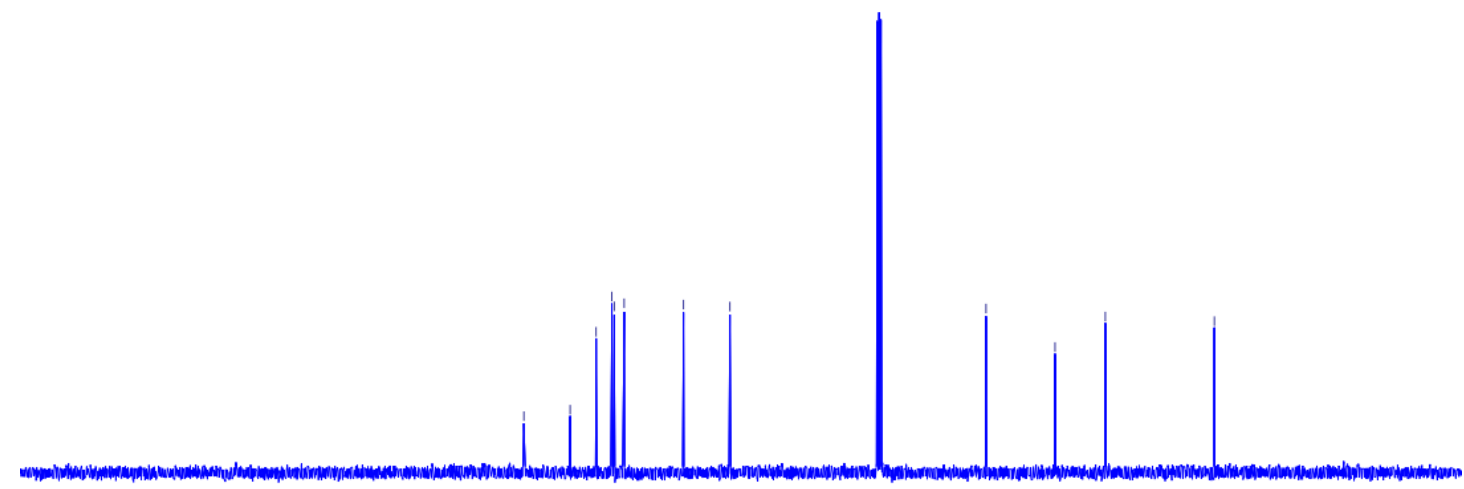

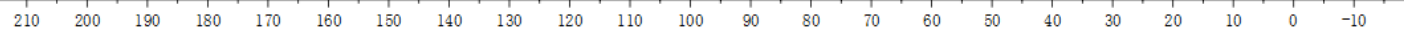

${ }^{1} \mathrm{H}$ NMR spectrum of compound $\mathbf{3 a b}\left(\mathrm{CDCl}_{3}, 400 \mathrm{MHz}\right)$

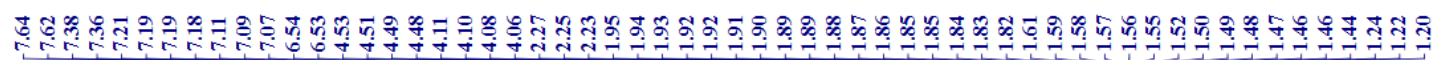

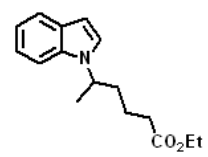

H NMR $400 \mathrm{MHz}, \mathrm{CDCl}_{3}$ )

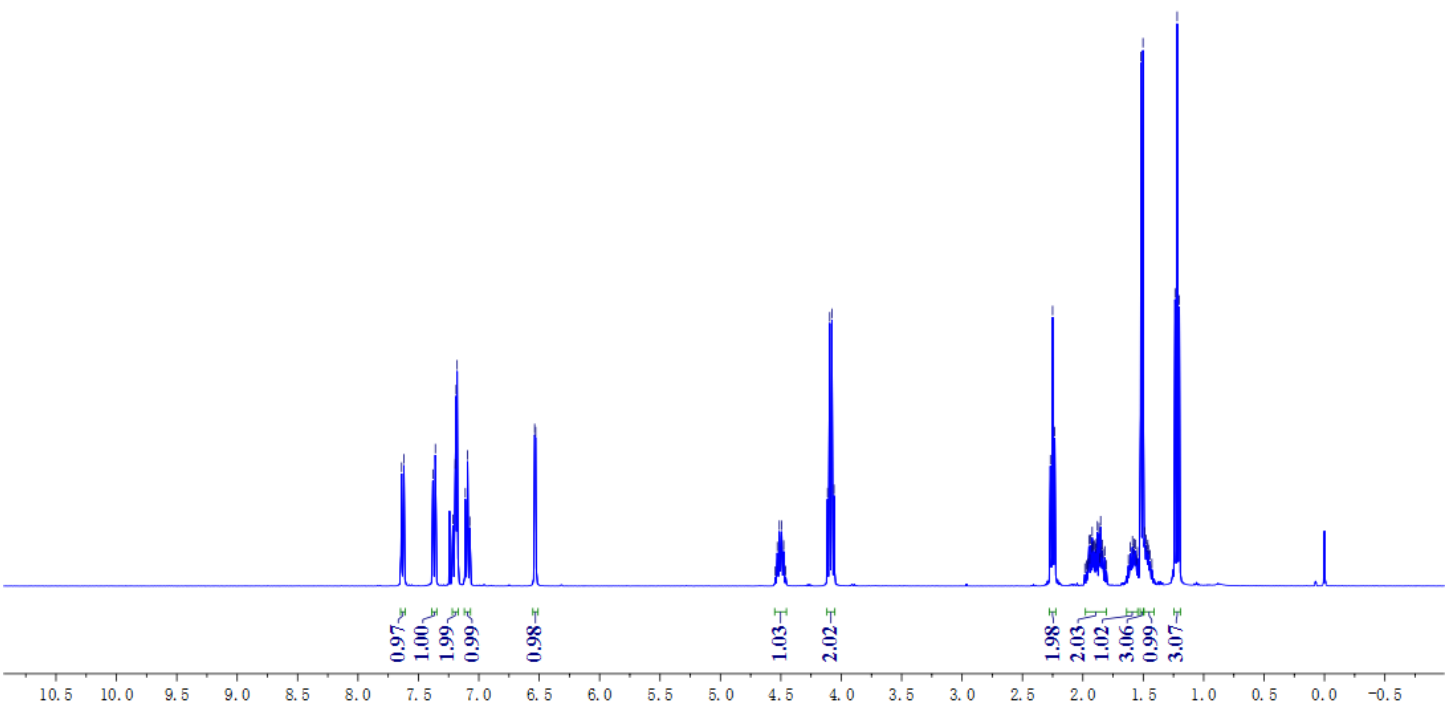


${ }^{13} \mathrm{C}$ NMR spectrum of compound $\mathbf{3 a b}\left(\mathrm{CDCl}_{3}, 101 \mathrm{MHz}\right)$
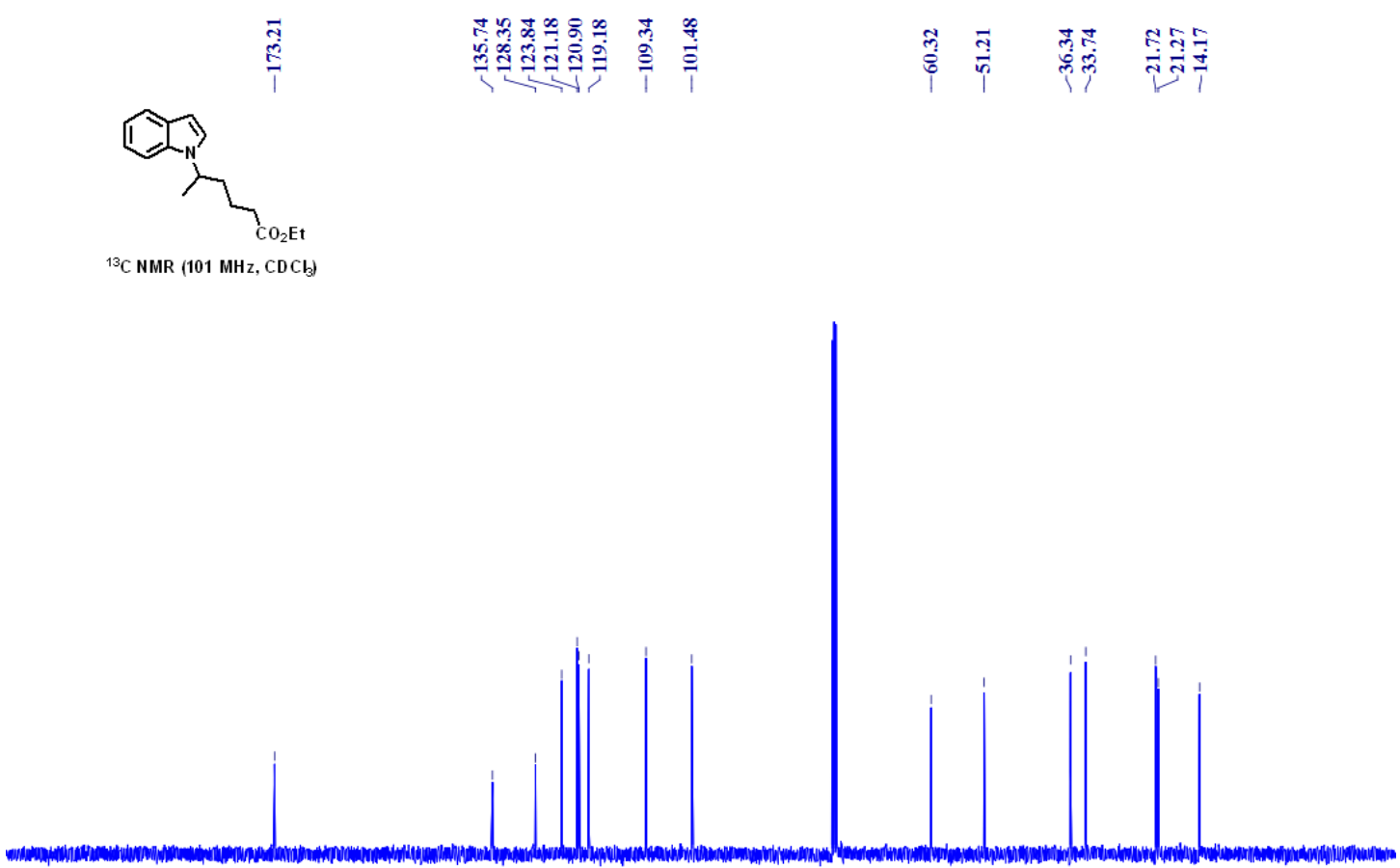

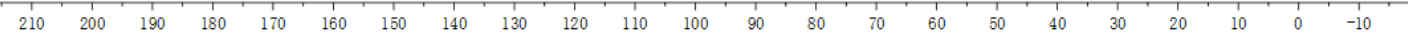

${ }^{1} \mathrm{H}$ NMR spectrum of compound $\mathbf{3 a c}\left(\mathrm{CDCl}_{3}, 400 \mathrm{MHz}\right)$

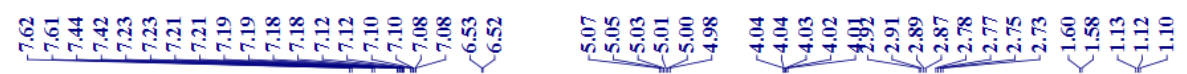

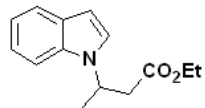

${ }^{1} \mathrm{H}$ NMR $\left(400 \mathrm{MHz}, \mathrm{CDCl}_{3}\right)$

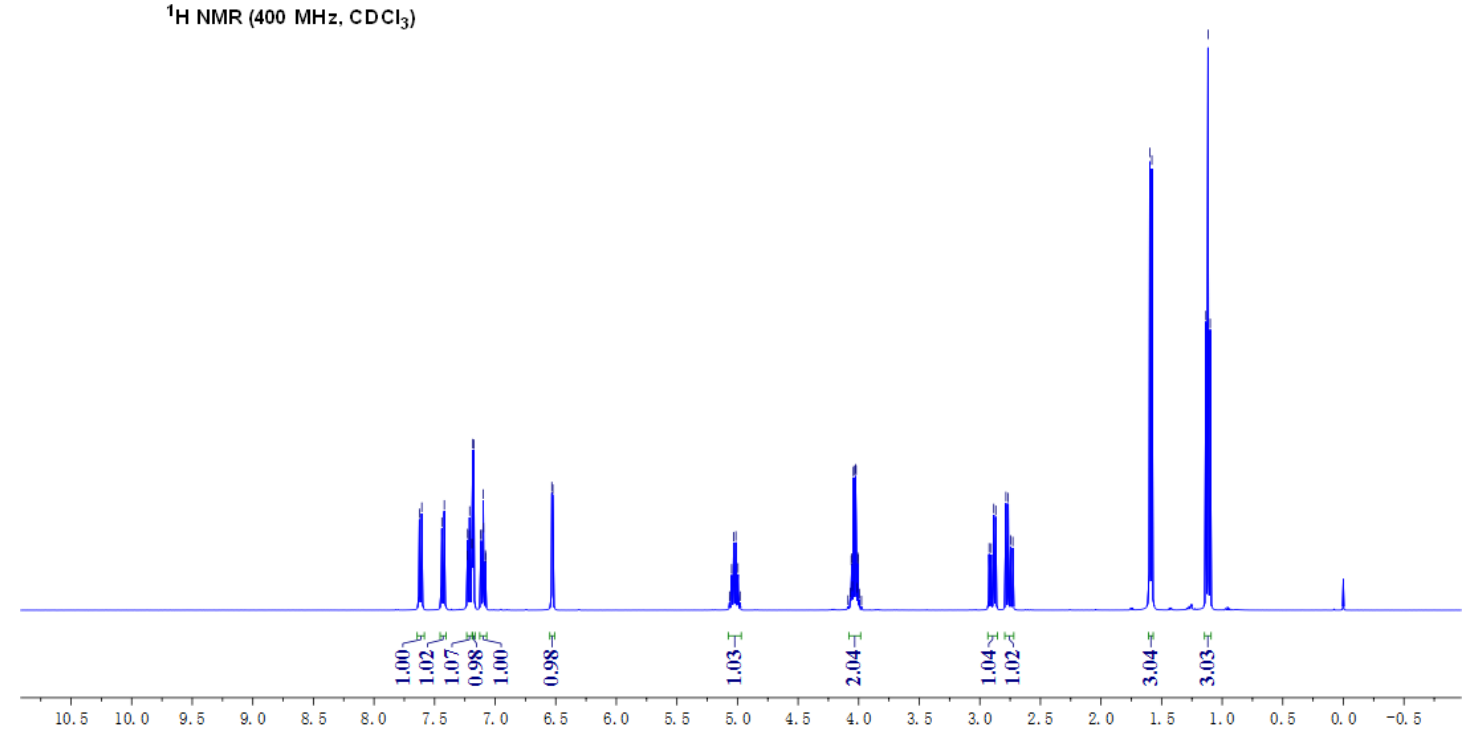


${ }^{13} \mathrm{C}$ NMR spectrum of compound $\mathbf{3 a c}\left(\mathrm{CDCl}_{3}, 101 \mathrm{MHz}\right)$

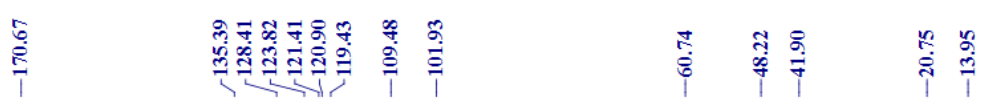

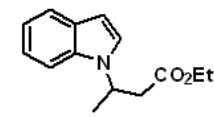

${ }^{13} \mathrm{C} \mathrm{NMR}\left(101 \mathrm{MHz}, \mathrm{CDCl}_{3}\right)$

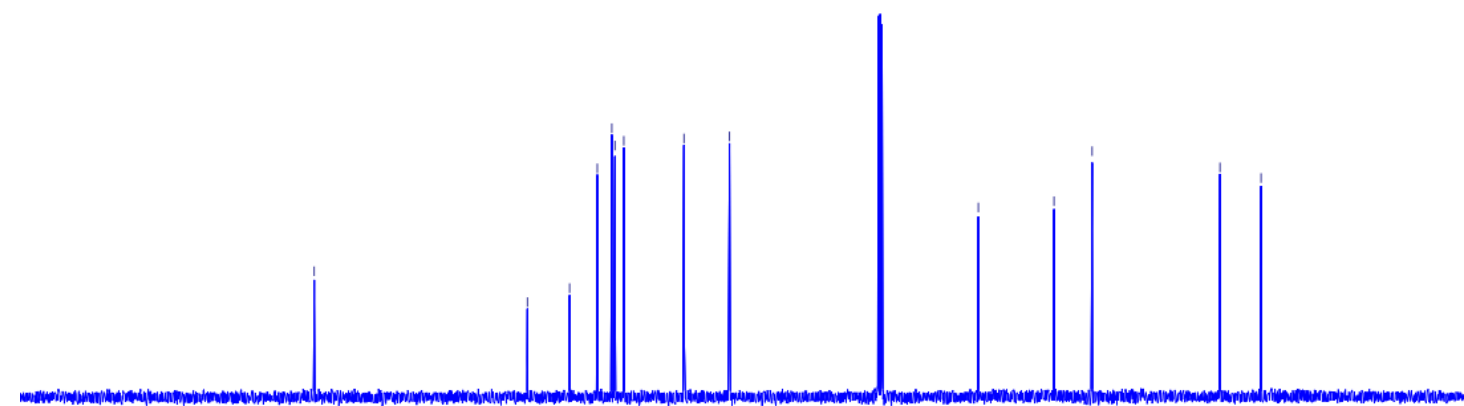

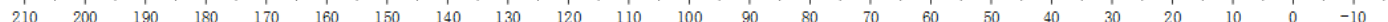

${ }^{1} \mathrm{H}$ NMR spectrum of compound $\mathbf{3 a d}\left(\mathrm{CDCl}_{3}, 400 \mathrm{MHz}\right)$

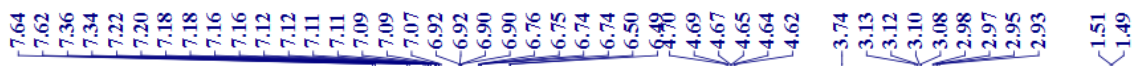

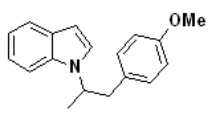

$1 \mathrm{HMR}(400 \mathrm{MHz}, \mathrm{CDCl}$

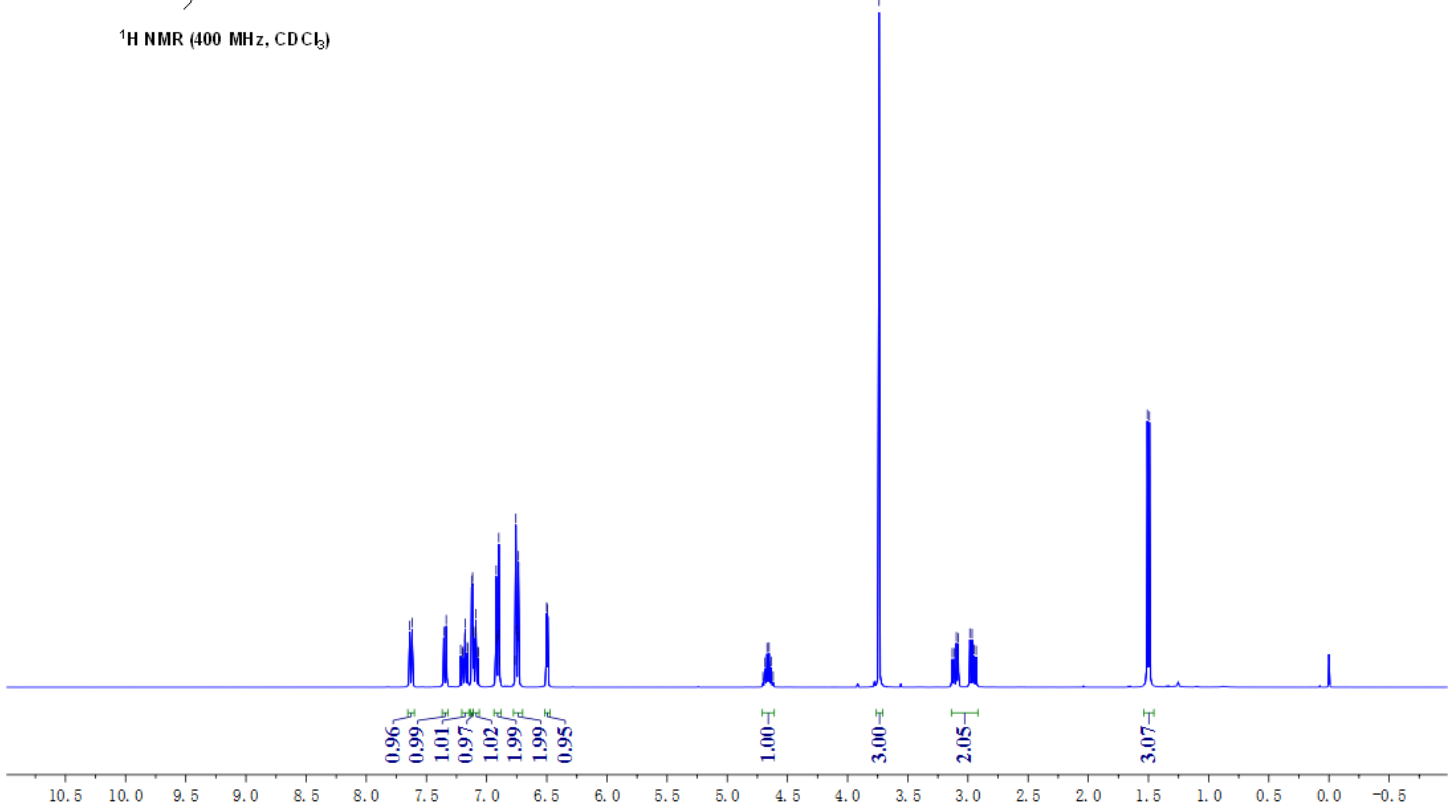


${ }^{13} \mathrm{C}$ NMR spectrum of compound $\mathbf{3 a d}\left(\mathrm{CDCl}_{3}, 101 \mathrm{MHz}\right)$
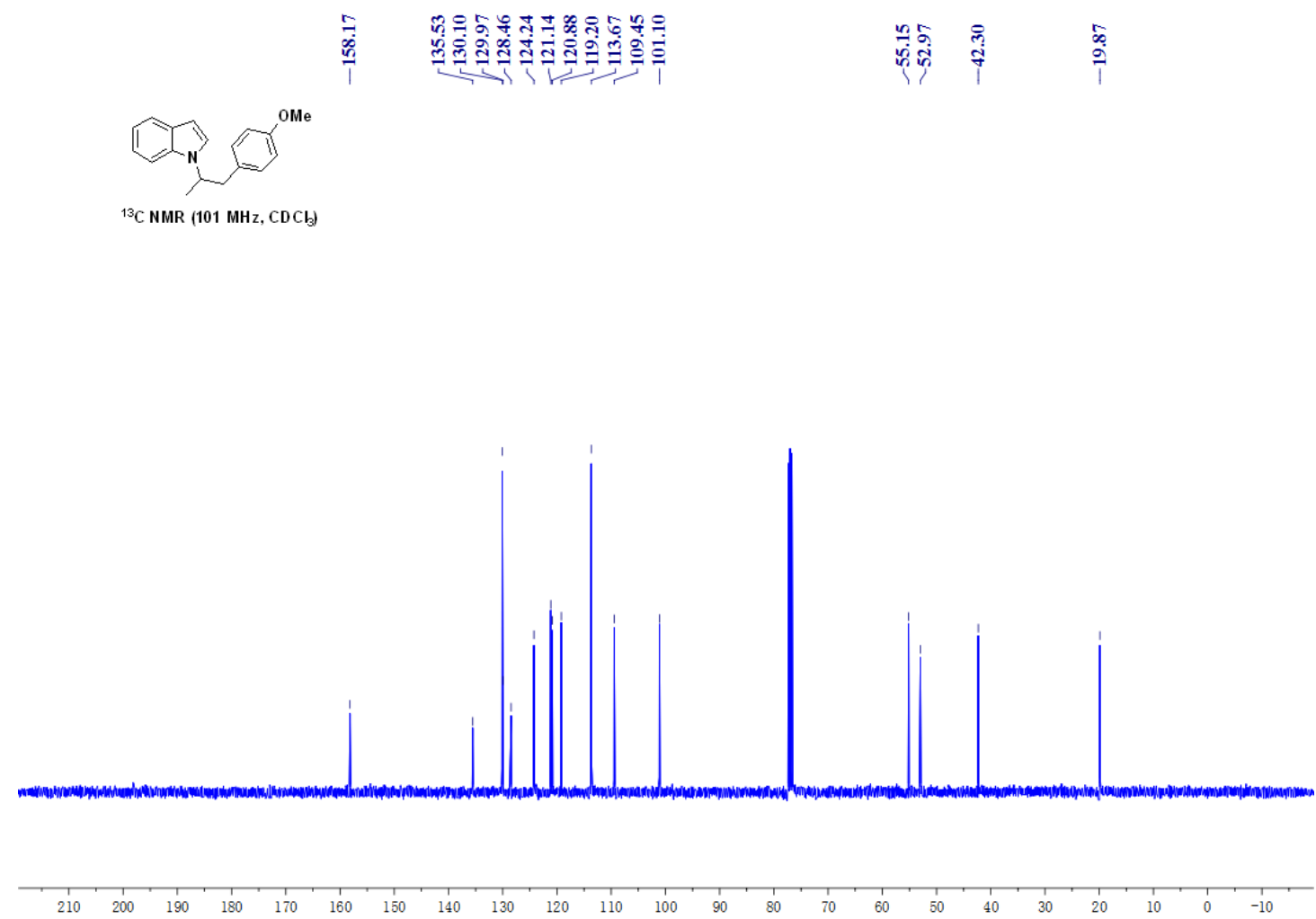

${ }^{1} \mathrm{H}$ NMR spectrum of compound $\mathbf{3 a e}\left(\mathrm{CDCl}_{3}, 400 \mathrm{MHz}\right)$

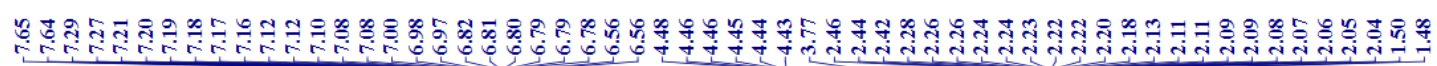

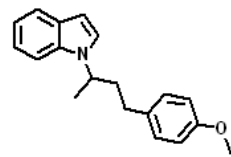

${ }^{1} \mathrm{H} \mathrm{NMR}\left(400 \mathrm{MHz}, \mathrm{CDCl}_{3}\right)$

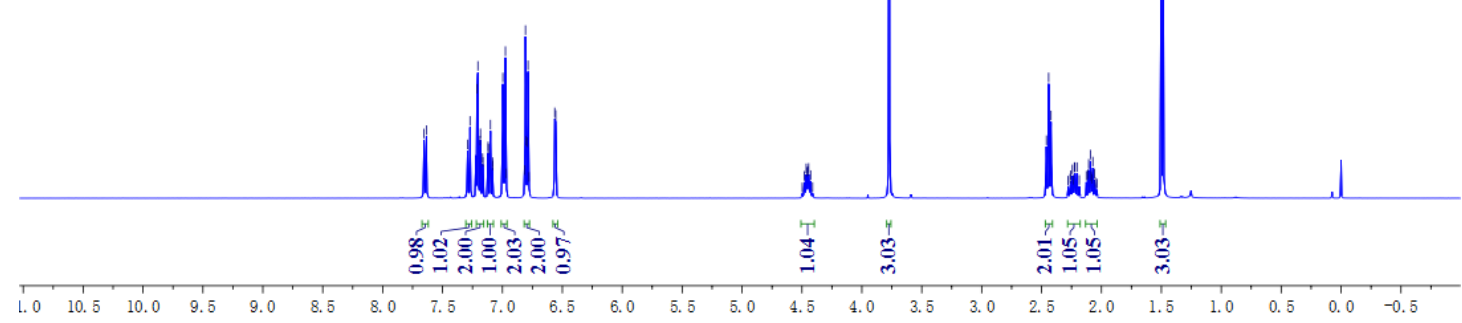


${ }^{13} \mathrm{C}$ NMR spectrum of compound 3ae $\left(\mathrm{CDCl}_{3}, 101 \mathrm{MHz}\right)$
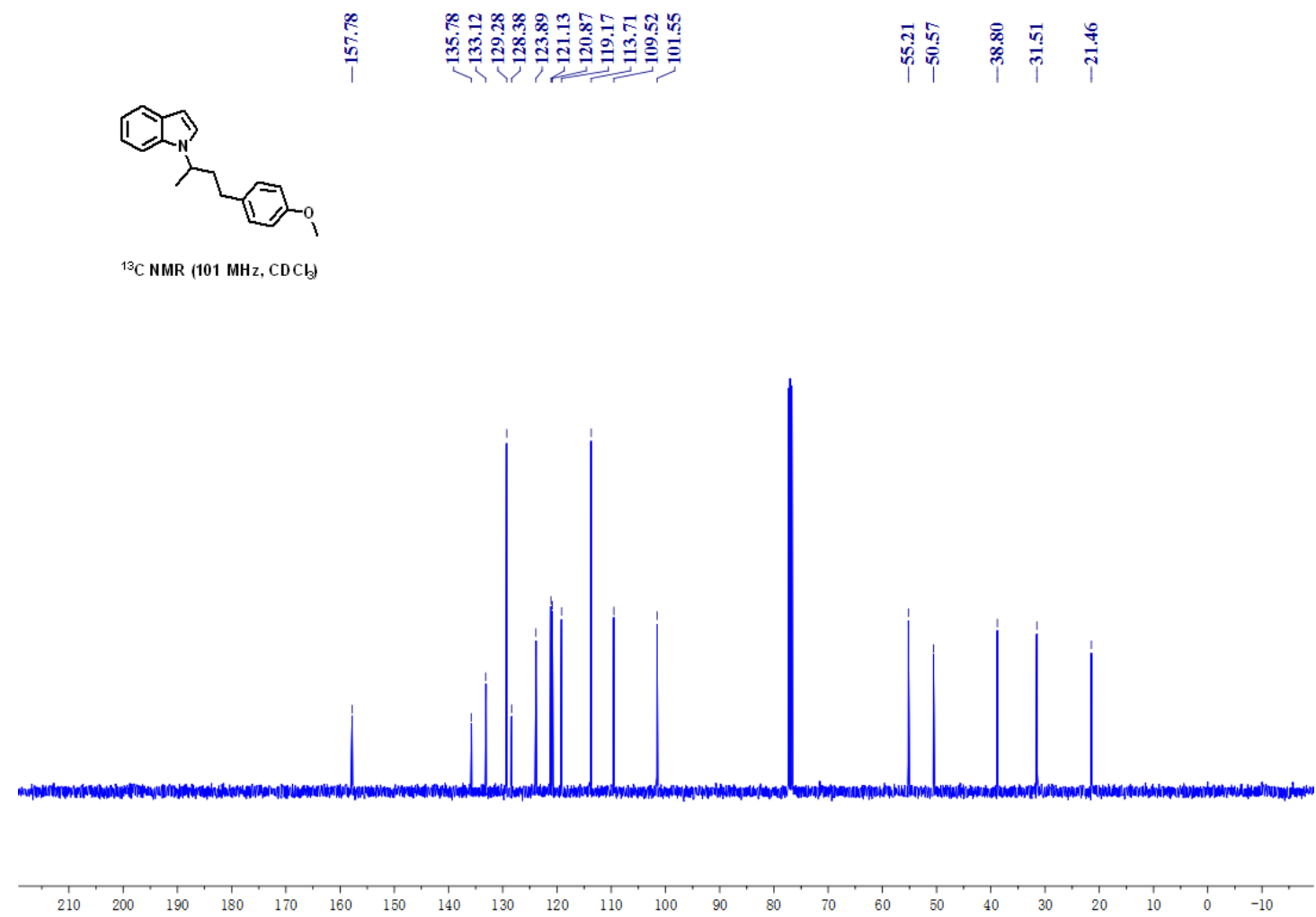

${ }^{1} \mathrm{H}$ NMR spectrum of compound 3af $\left(\mathrm{CDCl}_{3}, 600 \mathrm{MHz}\right)$

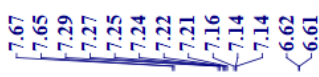

${ }^{1} \mathrm{H}$ NMR $\left(600 \mathrm{MHz}, \mathrm{CDCl}_{3}\right)$

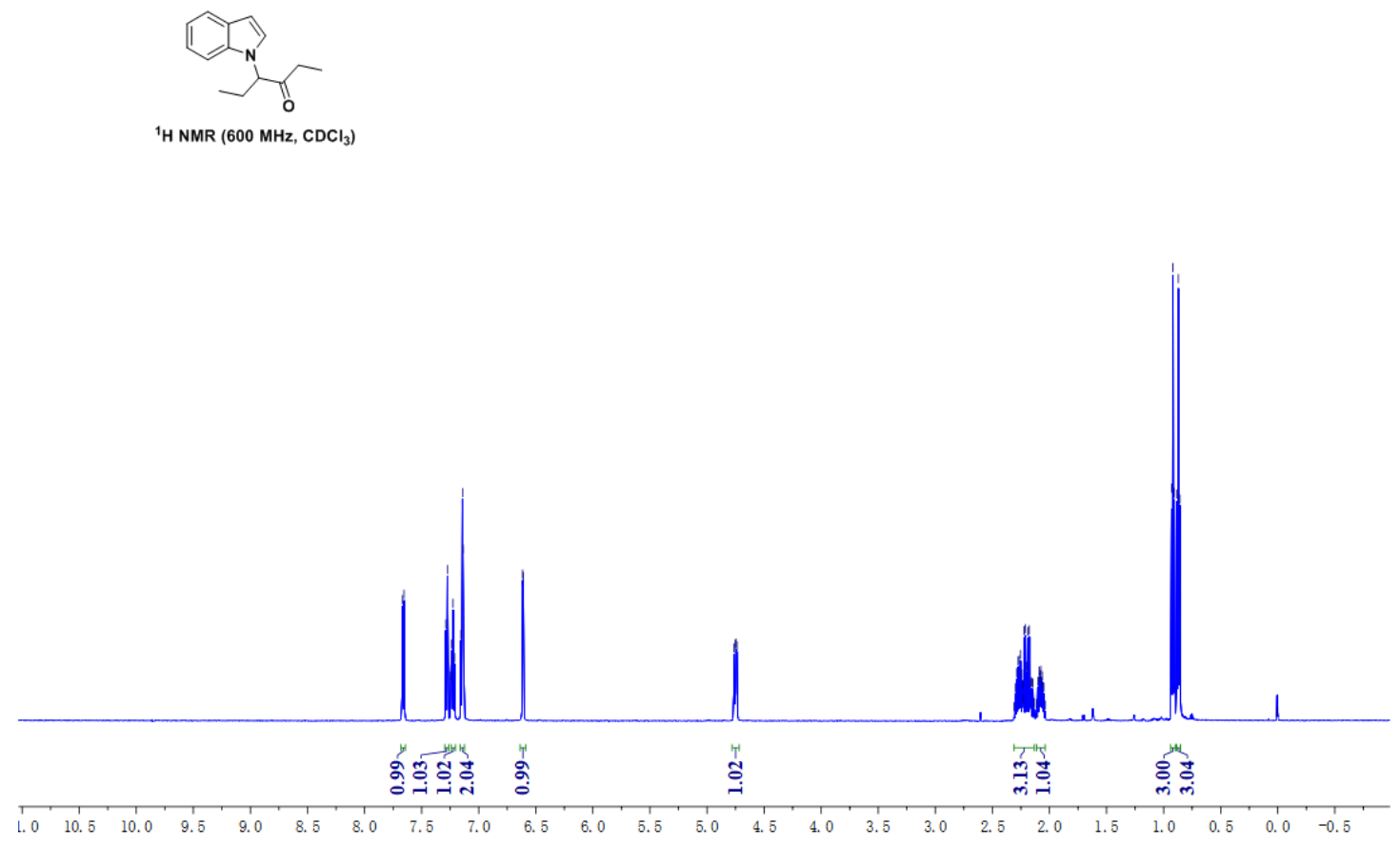

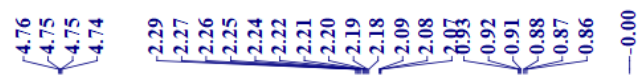

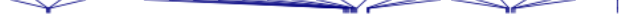


${ }^{13} \mathrm{C}$ NMR spectrum of compound 3af $\left(\mathrm{CDCl}_{3}, 151 \mathrm{MHz}\right)$

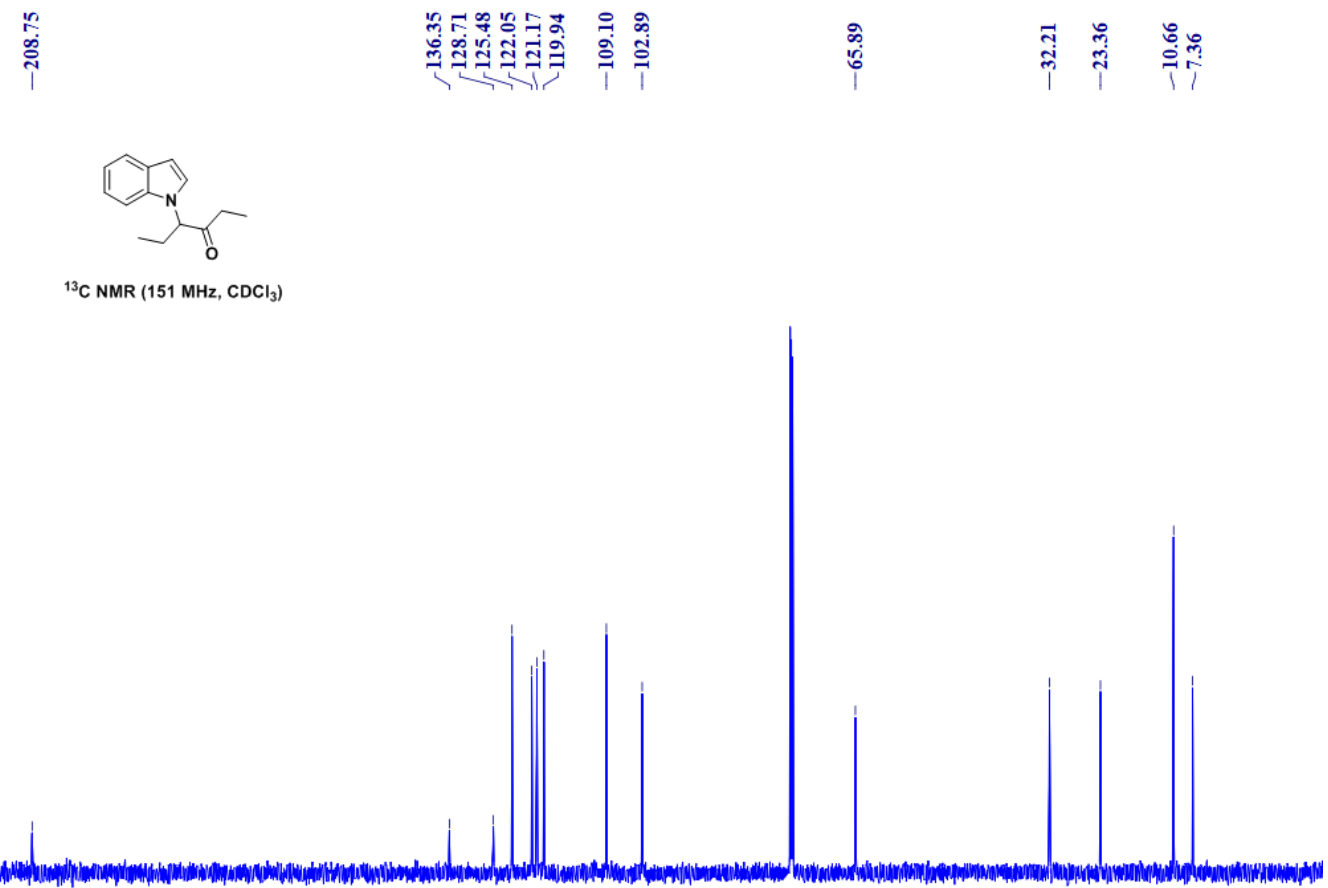

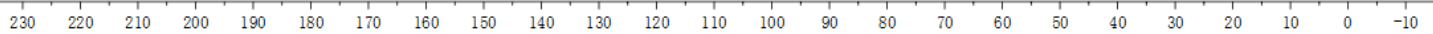

